\title{
EFFECT OF DE-CORRELATING TURBULENCE ON THE LOW FREQUENCY DECAY OF JET-SURFACE INTERACTION NOISE IN SUB-SONIC UNHEATED AIR JETS USING A CFD-BASED APPROACH
}

\author{
By \\ M. Z. Afsar \\ Imperial College London, 180 Queen's Gate, London, SW7, UK ${ }^{1}$ \\ S. J. Leib \\ Ohio Aerospace Institute, 22800 Cedar Point Road, Cleveland, Ohio 44142, USA \\ and \\ R. F. Bozak \\ National Aeornautics and Space Administration, Glenn Research Center, Cleveland, Ohio \\ 44135, USA
}

In this paper we extend the Rapid-distortion theory (RDT)-based model derived by Goldstein, Afsar \& Leib (J. Fluid Mech., vol. 736, pp. 532-569, 2013) for the sound generated by the interaction of a large-aspect-ratio rectangular jet with the trailing edge of a flat plate to include a more realistic upstream turbulence spectrum that possess a de-correlation (i.e. negative dip) in its space-time structure and use results from threedimensional Reynolds-Averaged Navier-Stokes (RANS) solutions to determine the mean flow, turbulent kinetic energy and turbulence length \& time scales. Since the interaction noise dominates the low-frequency portion of the spectrum, we use an appropriate asymptotic approximation for the Rayleigh equation Green's function, which enters the analysis, based on a two-dimensional mean flow representation for the jet. We use the model to predict jet-surface interaction noise for a range of subsonic acoustic Mach number jets, nozzle aspect ratios, streamwise and transverse trailing-edge locations and compare them with experimental data. The RANS meanflow computations are also compared with flow data for selected cases to assess their validity. We find that finite de-correlation in the turbulence spectrum increases the low-frequency algebraic decay (the lowfrequency "roll-off") of the acoustic spectrum with angular frequency to give a model that has a pure dipole frequency scaling. This gives better agreement with noise data compared to Goldstein et al. (2013) for Strouhal numbers less than the peak jet-surface interaction noise. For example, through sensitivity analysis we find that there is a difference of $10 \mathrm{~dB}$ at the lowest frequency for which data exists (relative to a model without decorrelation effects included) for the highest acoustic Mach number case. Secondly, our results for the planar flow theory provide a first estimate of the low-frequency amplification due to the jet-surface interaction for moderate aspect ratio nozzles when RANS meanflow quantities are used appropriately. This work will hopefully add to noise prediction efforts for aircraft configurations in which the exhaust systems are tightly integrated with the airframe.

\section{Nomenclature}

\footnotetext{
${ }^{1}$ Present address: Department of Mechanical \& Aerospace Engineering, Strathcylde University, 75 Montrose Street, Glasgow, G1 1XJ.
} 


$$
\begin{aligned}
& c_{\infty}=\text { ambient sound speed } \\
& D_{J} \quad=\text { nozzle diameter } \\
& g=\text { Green's function } \\
& I_{\omega} \quad=\text { acoustic spectrum } \\
& k=\text { turbulent kinetic energy } \\
& k_{1}=\text { streamwise wavenumber } \\
& l_{i}=\text { characteristic length scale } \\
& M \quad=\text { acoustic Mach number } \\
& p \quad=\text { pressure } \\
& t=\text { time } \\
& T=\text { averaging time } \\
& U_{c} \quad=\text { convection velocity } \\
& V=\text { source volume } \\
& v_{i}=\text { velocity vector } \\
& \boldsymbol{x}=\text { observer location } \\
& \boldsymbol{y}=\text { source location } \\
& \alpha=\text { turbulence intensity } \\
& \beta=\text { angle function } \\
& \gamma=\text { specific heat ratio } \\
& \varepsilon=\text { turbulence dissipation rate } \\
& \eta \quad=\text { separation vector } \\
& \rho=\text { density } \\
& \theta=\text { polar angle measured from jet axis } \\
& \tau=\text { time delay } \\
& \omega=\text { radian frequency } \\
& \omega_{c}=\text { convected scalar field } \\
& \nabla=\text { gradient operator } \\
& \text { | = absolute value }
\end{aligned}
$$

\section{Subscripts}

$\perp \quad=$ transverse component

Superscripts

$$
\begin{aligned}
& \text { - = } \quad \text { = time average } \\
& \text { = fluctuating quantity } \\
& \begin{aligned}
\sim & =\text { Favre average } \\
* \quad & =\text { complex conjugate }
\end{aligned}
\end{aligned}
$$


INTRODUCTION

Jet-surface interaction noise is a term that refers to the low-frequency amplification of sound that occurs when a high-speed air jet interacts with an external surface. For our purposes, we imagine the interaction taking place at the trailing edge of a flat plate that is positioned parallel to the oncoming flow ([1]-[3]). At the outset, however, we assume that jet-surface interaction consists almost entirely of trailing-edge noise in the test cases we consider. Indeed jet flows of technological interest are almost always close enough to, or sufficiently confined by, solid boundaries, such that the jet flow interacts with the edge of that boundary. Understanding how this interaction causes sound to be generated is of considerable importance for present-day and future aircraft that may have complex engine installation geometries ([4]-[10]).

The noise amplification associated with jet-surface interaction was investigated experimentally by Scharton et al. [11] in the context of aerofoil flap noise. Figure (13) in their paper indicates that the trailing edge interaction effect (with the flap alligned with the airfoil) is almost $10 \mathrm{~dB}$ greater than the jet noise at the peak frequency of the jet noise ninetydegree spectrum. The Scharton et al. experiment was at an acoustic Mach number ( $M a=U_{J} / c_{\infty}$, where $U_{J}$ is the jet exit velocity and $c_{\infty}$ is the ambient speed of sound) of about 0.5 (corresponding to a jet speed of $550 \mathrm{ft} / \mathrm{s}$ ) for a jet-aerofoil flap configuration, however, Head \& Fisher [12] obtained similar low frequency amplification results (see their Fig. 3) on a jet- surface interaction experiment with a more generic flat plate surface at the anechoic facility at the University of Southampton. The noise amplification they observed was also for an acoustic Mach number of 0.5 . In addition, they performed parametric studies of the near field spectra at different Mach numbers and shield separations (i.e. the transverse location of the plate relative to the jet centerline).

Similar experiments were carried out by Olsen \& Boldman [13] who confirmed that the presence of a leading/trailing edge from an external surface enhanced the noise produced by the jet alone for observation points on the same side as the jet flow. This result has now become well known and confirmed by many authors since; e.g. Wang ([14], Fig. 6) \& Southern ([15], Fig. 19) and also more recent references [16]-[18]. Even though much of past, and some of the recent, investigations have been performed on round jets interacting with trailing edges ([11], [15]--[18]), Head \& Fisher [12] noted that the observed results of noise amplification and trends with various jet parameters were similar to those produced by a large aspect ratio rectangular nozzle.

Experiments at the NASA Glenn Research Center ([1]-[3],[19]\&[20]) investigated the jetsurface interaction noise (as Head \& Fisher [12]) of a rectangular nozzle jet flow interacting with the trailing edge of an external plate. In the experiments reported in [1]-[3] and Zaman et al. [19], the power spectral density (PSD) of the far-field fluctuating pressure was measured for unheated, high Reynolds number, jet flows across a range of acoustic Mach 
numbers when the trailing edge was positioned above/beneath the flow at various axial/radial locations relative to the nozzle center line. The findings of [1]-[3] have generally confirmed the trends of previous studies [11]-[18]. In particular, at low frequencies, the PSD is considerably amplified compared to the free jet, and that this effect is greatest at large polar observation angles to the jet axis (i.e. near 90 )-see Fig. 8 in [3] where it is illustrated that the jet-surface interaction effect is between 10-20 dB at the peak Strouhal number (see also p. 16 in Brown [20]) relative to the jet noise (similar conclusions were reached by Scharton et al. [11]).

Figures 6 \& 7 in Head \& Fisher [12] indicate that the trailing edge noise 'source' can be thought of as an acoustic dipole at the trailing edge with dipole axis perpendicular to the plate. Note that the experimental measurements are likely to display the ideal symmetry of a point dipole (see Howe [21], p. 217) at the trailing edge only in the absence of interference effects of the edge noise with jet noise.

The fact that the trailing edge noise source is a much more acoustically efficient radiator at lower Mach numbers was also confirmed by Bridges et al. [1]. For example, Fig. 12 in Bridges [3], indicates that the difference between the total measured noise (with trailing edge) and the isolated jet noise is greater at lower acoustic Mach numbers. This effect is more prominent when the distance from the plate trailing edge location to nozzle exit plane is increased (see Fig. 8 in Bridges [3] and also p.299 \& Fig. 11a in [14]). It is also clear from Fig. 13 in Scharton et al. [11] that the peak jet surface interaction noise occurs at a slightly lower frequency than the peak jet noise.

In references [13], [22]\&[23], and more recently in [24] (hereafter referred to as GAL), it was shown that mean flow effects are needed to accurately predict the amplification level of the far-field sound when an external surface in placed near a jet. Any model that neglect this effect (such as applications of [25]) will never be entirely predictive. In [20], Brown developed an empirical model for jet-surface interaction noise and extracted the noise due to the jet/trailing edge interaction from measurements of the total noise in various jet-plate configurations using assumed dipole characteristics of the edge noise source. Such empirical models may be incorporated into system-level studies to assess the noise impact on various engine installation configurations, however, they are unlikely to more be general than the parameter space in which they are derived and do not give insight into the physics.

A prediction method based on a self-consistent application of the non-homogeneous Rapid-distortion theory (RDT) was introduced in [24] and an initial application of the general theory to the jet-surface interaction problem was demonstrated. RDT is the study of small amplitude turbulent fluid motion that, owing to some sort of 'distortion', evolves linearly about a mean flow. In classical RDT ([26]--[33]), the upstream flow is taken to be uniform, which, therefore, allows a vortical disturbance that is frozen in the flow to be used as a boundary condition for the interaction problem ([30], [31] and p. 569f. of [32]). The aim of this paper is to further develop the RDT-based prediction method of [24] for the noise generated by the interaction of a turbulent jet with the trailing edge of a flat plate. It is 
expected that sound generated by the turbulence convecting past the trailing edge exceeds the non-linear sub-sonic jet noise (see [34]) and the jet-surface scrubbing noise (e.g. as formulated by Khavaran [35]) in the test cases considered. This is because the jet noise source (i.e. the fluctuating Reynolds stress) is quadratic in velocity fluctuation, whereas edge-noise source is linear [34]. Brown ([1] , p. 7) actually indicates that since the scrubbing noise is blocked by the surface in the shielded location (plate between jet and observer), its magnitude can be assessed by comparing the measurements below to that above the plate (reflected location). In experiments on an axi-symmetric round jet (fig. 10 of [1]) and non-axisymmetric jet from a rectangular nozzle [3], the data shows this difference is often less than $5 \mathrm{~dB}$ for high acoustic Mach number isothermal flow over the Strouhal number range over which noise amplification is observed when the trailing-edge suitably located relative to the nozzle exit plane. Given that the amplification itself is of the order of $10-20 \mathrm{~dB}$ (see previous citations), the restriction to the trailing-edge component would seem appropriate.

Our objectives here are to introduce a more realistic model for the statistics of the upstream turbulence and using a Reynolds Averaged Navier-Stokes (RANS) Computational Fluid Dynamics (CFD) solution to determine the mean flow and inform the source model by providing the appropriate turbulent length scales and source amplitude. The use of RANS solutions also allows these flow quantities to vary with flow conditions and geometry. The RANS solutions are obtained using the SolidWorks ${ }^{\circledR}$ Flow Simulation software [36]\&[37], which provides relatively fast solutions for the geometries of interest. As a check on the quality of these results, we include comparisons with measured flow data [19] for selected cases. The modelling approach we take is similar to how modern acoustic analogy approaches [38] use CFD data, however, in our case, only certain components of the upstream turbulence (that enters through the second-order transverse velocity auto covariance) appear in the acoustic spectrum formula. Our results show that a negative dip (or 'de-correlation') in this quantity directly impacts the low-frequency algebraic decay (often referred to as the 'roll-off') of the edge-generated noise to give an acoustic spectrum that has a pure dipole pre-factor for all frequencies over which the sound amplification occurs. This model generally provides better agreement with experimental data than our previous results. The presence of such negative regions in the second-order correlations of the transverse velocity field (i.e. perperdicular to the flow direction) has been known for some time [39]\&[40]. This suggests that manipulation of the upstream turbulence could be exploited for trailing-edge noise control strategies that are different from existing methods such as serrated edges (see, [41]--[42] and [43]--[44] for leading edges) or wall turbulence control methods [45].

Alternative approaches to jet-surface interaction noise have used Amiet's theory [46] as the starting point (see, for example, Miller [47]). Other efforts have focused more on aerofoil applications [48] using Goldstein's (1978) RDT theory [22] to model high frequency noise at low Mach numbers. There have also been a number of numerical studies (Wolf et al.[49], Cheung et 
al. [50] and Venugopal et al. [51]) in which Large-Eddy simulations were used to predict aerofoil noise and jet-surface interaction with a rectangular jet and flat plate [52].

The paper starts with a short review of the relevant parts of the GAL analysis used in this work. In Section 3, we introduce the new model for the transverse velocity correlations and present the corresponding formula for the acoustic spectrum. In Section 4 we illustrate some generic features of the model using an analytical mean flow model given by Eq. (6.55) in GAL. The impact of the new turbulence model on the low-frequency roll-off of the spectrum and comparions with the model of GAL are shown. These results illustrate that: a) the far field noise predictions based on the planar flow (i.e. large aspect ratio) theory are more accurate compared to our 2013 results at frequencies less than the peak when the upstream turbulence de-correlates; and b) this large aspect ratio theory gives noise predictions that are reasonable first estimate for non-planar jet flows (i.e. within a band of about $5 \mathrm{~dB}$ between the smallest and largest aspect ratio nozzles). This reduces to less than $2 \mathrm{~dB}$ for the highest Mach number considered here $(M a=0.9)$ at the upper frequency limit of interaction noise $(S t \sim 0.5)$. Neither of these aspects of jet-suface interaction were considered by GAL. In Section 5 we describe the SolidWorks ${ }^{\circledR}$ RANS solutions used in this work and compare them with experimental data taken at NASA Glenn [19] for the mean flow and turbulent kinetic energy distributions near the edge of the plate.

In Section 6 we present extensive comparisons of noise predictions with experimental data using the RDT-based model developed in this paper, with SolidWorks RANS solutions for the mean flow and turbulence properties. The edge-noise data is taken to be the change in the measured sound radiation relative to the jet noise without a plate. The results show improved prediction over Mach number and polar observation angle when the streamwise mean velocity component, as well as the local turbulent kinetic energy and rate of energy dissipation, are obtained from a RANS calculation. Notwithstanding that the theory strictly applies to a planar mean flow, we use it (as above) as an approximation to a non-planar flow for a lower aspect ratio rectangular jet interacting with a trailing edge. We, however, vary the upstream turbulence properties to compensate for their expected variation with jet aspect ratio. Note that our predictions are compared mainly against acoustic measurements in the forward arc since, for the test cases we consider, this region showed the most significant sound amplification (i.e. $\sim 10 \mathrm{~dB}$ or greater). Hopefully, our work will add to efforts aimed at incorporating Computational Fluid Dynamics (CFD)-obtained mean flows systematically within an analytical approach for jet-surface interaction noise (see, for example [53]-[57]).

\section{REVIEW OF THE GOLDSTEIN, AFSAR \& LEIB (GAL [24]) FORMULATION}

\subsection{Distinguished limit for rapidly-distorting non-homogeneous turbulence}

The solution of the basic RDT equations in GAL can be used for any interaction problem involving non-homogeneous turbulence undergoing rapid-distortion on a nearly parallel 
mean flow. For the jet-surface interaction noise problem, for example, to which we shall apply this theory, it is assumed that the maximum sound generated at the edge exceeds the (non-linear) jet noise owing to the fact that the jet noise source strength is quadratic in the small velocity fluctuations (see [34]) at the trailing edge while the 'RDT source' is linear in those small fluctuations.

To illustrate the basic scalings, we allow lengths and velocities to be non-dimensionalized by $D_{J}$ and $U_{J}$ respectively; time by $D_{J} / U_{J}$ and pressure by $\rho_{J} U_{J}^{2}$, where $\rho_{J}$ and $U_{J}$ are flow density and velocity, respectively, at the nozzle exit and $D_{J}$ is an appropriate reference length scale (such as the nozzle exit equivalent diameter for a rectangular jet). The flow Reynolds number is assumed to be large, i.e. $\mathrm{R}=U_{J} D_{J} / v \gg O(1)$, where $v$ is the kinematic viscosity, and the turbulence Reynolds number during the distortion is as high as order 1 (i.e. $\left.\mathrm{R}_{T}=\alpha \mathrm{R}=O(1)\right)$, where $\alpha \equiv|\boldsymbol{u}| / U_{J} \ll O(1)$ is the turbulence intensity of the flow near where the interaction takes place (i.e. the trailing edge in our problem) and $|\boldsymbol{u}|$ is the magnitude of the local rms turbulence velocity. This is the distinguished limit for nonhomogeneous turbulence (representative of jet flows) undergoing a sudden interaction ([29]\&[34])

Physically, this distinguished limit describes the change in small-amplitude turbulence near the trailing egde within a time interval shorter than a characteristic eddy decay time, $\tau_{\text {int }}<l /|\boldsymbol{u}|$ (where $l$ is an appropriate length scale). As indicated in [34], the turbulence Reynolds is then usually large (relative to unity) and the unsteady flow is determined by the invsicid compressible Rayleigh equation on a transversely sheared flow (Eqs. 2.2 \& 2.3 in GAL) since any turbulent shear flow will be transverely sheared over the stream wise distances where RDT is valid.

\subsection{The fundamental RDT solutions}

The spatial field point is denoted by the Cartesian co-ordinates $\boldsymbol{y}=\left\{y_{1}, y_{2}, y_{3}\right\}=\left\{y_{1}, \boldsymbol{y}_{T}\right\}$ with transverse co-ordinates, $\boldsymbol{y}_{T}=\left\{y_{2}, y_{3}\right\}$ and origin is placed at the trailing edge of flat plate. The convective derivative, $D_{0} / D \tau \equiv \partial / \partial \tau+U \partial / \partial y_{1}$, follows material particles along level curves of a transversely sheared mean flow, $U\left(\boldsymbol{y}_{T}\right)=$ const. Following GAL we suppose that the flow is non-heat conducting and isentropic, as well as satisfying the ideal gas law. The solutions for the inviscid pressure perturbation, $p^{\prime}=p-p_{0}$ (where $p_{0}=$ constant) and momentum flux perturbation $u_{i} \equiv \rho v_{i}^{\prime}$, (where $v_{i}^{\prime}$ denotes the velocity perturbation) are then given by integral solutions:

$$
p^{\prime}(\boldsymbol{x}, t)=-\int_{-T}^{T} \int_{V(\boldsymbol{y})} \frac{D_{0}^{3} g}{D t^{3}}(\boldsymbol{y}, \tau \mid \boldsymbol{x}, t) \tilde{\boldsymbol{\omega}}_{c}\left(\tau-y_{1} / U\left(\boldsymbol{y}_{T}\right), \boldsymbol{y}_{T}\right) d \boldsymbol{y} d \tau
$$


and

$$
\rho v_{\perp}^{\prime}(\boldsymbol{x}, t)=-\frac{\partial U / \partial x_{i}}{|\nabla U|} \int_{-T}^{T} \int_{V(\boldsymbol{y})} g_{i}(\boldsymbol{y}, \tau \mid \boldsymbol{x}, t) \tilde{\omega}_{c}\left(\tau-y_{1} / U\left(\boldsymbol{y}_{T}\right), \boldsymbol{y}_{T}\right) d \boldsymbol{y} d \tau,
$$

where $\rho v_{\perp}^{\prime} \equiv \rho v_{i}^{\prime}\left(\partial U / \partial x_{i} /|\nabla U|\right)$ and $T$ denotes a very large (but finite) time interval.

In Eq. (2), $g_{i}(\boldsymbol{y}, \tau \mid \boldsymbol{x}, t)$, defined through,

$$
g_{i}(\boldsymbol{y}, \tau \mid \boldsymbol{x}, t) \equiv \frac{D_{0}}{D t}\left(\frac{\partial}{\partial x_{i}} \frac{D_{0}}{D t}+2 \frac{\partial U}{\partial x_{i}} \frac{\partial}{\partial x_{1}}\right) g(\boldsymbol{y}, \tau \mid \boldsymbol{x}, t)
$$

is linearly related to the Rayleigh equation Green's function:

$$
\begin{aligned}
& L(\boldsymbol{y}, \tau) g(\boldsymbol{y}, \tau \mid \boldsymbol{x}, t) \equiv \\
& \quad\left\{\frac{D_{0}}{D \tau}\left(\frac{\partial}{\partial y_{i}} c^{2} \frac{\partial}{\partial y_{i}}-\frac{D_{0}^{2}}{D \tau^{2}}\right)-2 \frac{\partial U}{\partial y_{j}} \frac{\partial}{\partial y_{1}} c^{2} \frac{\partial}{\partial y_{j}}\right\} g(\boldsymbol{y}, \tau \mid \boldsymbol{x}, t)=\delta(\boldsymbol{y}-\boldsymbol{x}) \delta(\tau-t)
\end{aligned}
$$

where $c^{2}\left(\boldsymbol{y}_{T}\right)$ denotes the mean sound speed squared and $g(\boldsymbol{y}, \tau \mid \boldsymbol{x}, t)$ is determined for incoming wave behavior as $|\boldsymbol{y}| \rightarrow \infty$ and appropriate boundary conditions on the bounding surfaces $S(y)$ for integral solutions, Eqs. (1) \& (2), to hold. The integrals in these results involve the arbitrary, purely convected, quantity $\tilde{\omega}_{c}\left(\tau-y_{1} / U\left(\boldsymbol{y}_{T}\right), \boldsymbol{y}_{T}\right)$ (satisfying $\left.D_{0} \tilde{\omega}_{c} / D \tau \equiv 0\right)$ that can be used to specify the 'input' within a boundary value problem (BVP) that seeks to determine acoustic spectrum as its 'output'.

The above solutions are valid for $M a=O(1)$ subsonic Mach numbers in un-heated jets. Heated jets could be considered by including enthalpy fluctuations appropriately into the energy equation. However we do not pursue that here and restrict the analysis to unheated jet flows in which the free-stream Mach number is zero. The solid surfaces $S(\boldsymbol{y})$ bounding volume $V(y)$ in (3) can be finite, semi-infinite or infinite in the streamwise direction and are assumed to coincide with one of the level surfaces of the mean velocity profile (since this is the arrangement of the simplest jet-surface interaction experiments reported in [1]-[3]).

\subsection{Green's function splitting for input-output problem}

The solution approach simply involves using the Rayleigh equation to calculate the unsteady flow for a prescribed upstream state (referred as the 'gust solution') as well as the far-field sound that it generates. GAL explain how dividing the Rayleigh equation Green's 
function that appears in Eqs. (1) \& (2) into two components as, $g=g^{(0)}+g^{(s)}$ where superscipt ${ }^{(0)}$ refers to the input gust solution and ${ }^{(s)}$ is the (output) scattered field can accomplish this. The gust-associated Green's function is now defined on all space and satisfies

$$
\hat{n}_{i} \partial\left[D_{0}^{3} g^{(0)}(\boldsymbol{y}, \tau \mid \boldsymbol{x}, t) / D t^{3}\right] / \partial y_{i}=0 \quad \text { for } \boldsymbol{y}_{T} \in S(\boldsymbol{y})
$$

on a doubly infinite surface $S(\boldsymbol{y})$, whose outward-drawn unit normal vector is $\hat{\boldsymbol{n}}(\boldsymbol{y})=\left\{\hat{n}_{i}\right\}(\boldsymbol{y})$, and is appropriate for defining a non-radiating input disturbance whose interaction with a discontinuity of this surface (e.g., the edge of a semi-infinte flat plate) produces a downstream response. Then, by Eq. (2), the corresponding transverse momentum flux $\rho v_{\perp}^{\prime}(\boldsymbol{x}, t)$ associated with the gust is given by,

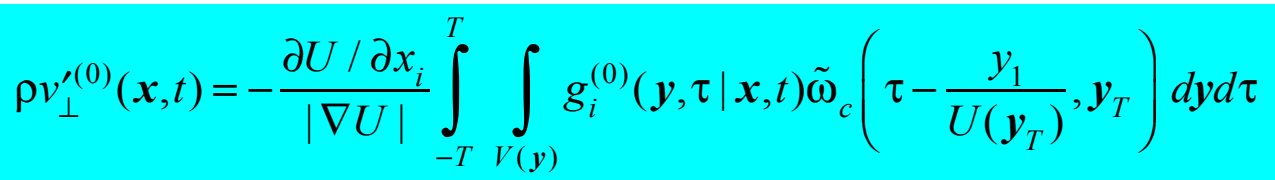

Eq. (6) together with Eq. (3) (when expressed in terms of $g^{(0)}$ ) then provides an integral relation between $\tilde{\omega}_{c}$ and the transverse momentum flux perturbation, $\rho v_{\perp}^{(0)}$, which is a physical quantity that could be measured experimentally or computed from a Large Eddy Simulation (LES). This relation can, in principle, be inverted (using identity Eq. (1.97) in [58]) to solve for the Fourier transform of $\tilde{\omega}_{c}$ in terms of the Fourier transform of $\rho v_{\perp}^{(0)}$.

\subsection{Relation between the $\tilde{\omega}_{c}$ spectrum and measurable turbulence statistics}

As in GAL, it is assumed that the relation between $\tilde{\omega}_{c}$ and $\rho v_{\perp}^{(0)}$ in the actual flow is the same as it would in an idealized mathematical representation of a transversely sheared mean flow $U\left(\boldsymbol{y}_{T}\right)$ in which the bounding surfaces present are doubly infinite in the streamwise direction (i.e., where the transverse boundary conditions are completely uniform in $y_{1}$ ). Hence the upstream boundary condition determined on this streamwisehomogeneous flow is assumed to be the same as that in the vicinity of trailing-edge where the distortion actually takes place. In this case, as pointed out by GAL, the unsteady flow generated by Eq. (6) (or Eq. (1) when expressed in terms of $g^{(0)}$ ) does not propagate to the farfield at subsonic speeds since the its streamwise Fourier transform (Eq. 4.6 in GAL) exists at the (hydrodynamic) wavenumber, $k_{1}=\omega / U\left(y_{2}\right)$, rather than propagating acoustic wavenumber, $k_{1}=\omega / c_{\infty}$ (this was emphasised in [59]; see also Fig. 1 on p. 344 and p. 284 in [60]). 
GAL used Eqs. (3) \& (6) to obtain an approximate relation between the Fourier transforms of $\rho v_{\perp}^{\prime(0)}$ and $\tilde{\omega}_{c}$ that can be used to obtain a relatively simple working formula relating the spectrum of the convected quantity $\tilde{\omega}_{c}$ to that of $\rho v_{\perp}^{\prime(0)}$ for a two-dimensional mean flow. The details are worked out in GAL (pp. 556-558) and we simply quote their result to be used in our re-analysis of the problem; hence the spectrum,

$$
S\left(y_{2}, \tilde{y}_{2} ; k_{3}, \omega ; y_{d}\right) \equiv \frac{1}{2 \pi} \int_{-\infty}^{\infty} \int_{-\infty}^{\infty} e^{i\left(\omega \tau-k_{3} \eta_{3}\right)}\left\langle\tilde{\omega}_{c}\left(t, y_{2}, y_{3}\right) \tilde{\omega}_{c}\left(t+\tau, \tilde{y}_{2}, y_{3}+\eta_{3}\right)\right\rangle d \tau d \eta_{3},
$$

is related to the experimentally measurable transverse velocity spectrum

$$
F_{\perp}\left(x_{2}, \tilde{x}_{2} \mid y_{2}, \tilde{y}_{2}, \omega, k_{3}\right)=\frac{1}{(2 \pi)^{2}} \int_{-\infty}^{\infty} \int_{-\infty}^{\infty} e^{-i\left(\omega \tau-k_{3} \eta_{3}\right)} f_{\perp}\left(x_{2}, \tilde{x}_{2} \mid \omega / U\left(y_{2}\right), \omega / U\left(\tilde{y}_{2}\right), \eta_{3}, \tau\right) d \eta_{3} d \tau
$$

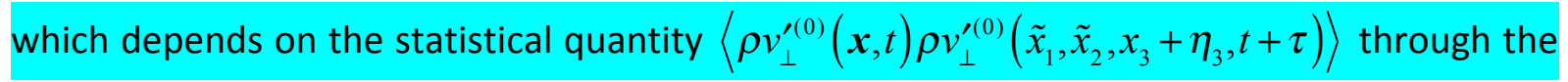
streamwise Fourier transforms

$$
f_{\perp}\left(x_{2}, \tilde{x}_{2} \mid k_{1}, \tilde{k}_{1}, \eta_{3}, \tau\right) \equiv \frac{1}{(2 \pi)^{2}} \int_{-\infty}^{\infty} \int_{-\infty}^{\infty} e^{-i\left(x_{1} k_{1}-\tilde{x}_{1} \tilde{k}_{1}\right)}\left\langle\rho v_{\perp}^{\prime(0)}(\boldsymbol{x}, t) \rho v_{\perp}^{\prime(0)}\left(\tilde{x}_{1}, \tilde{x}_{2}, x_{3}+\eta_{3}, t+\tau\right)\right\rangle d x_{1} d \tilde{x}_{1},
$$

where $\langle\bullet\rangle$ is the time and spanwise average of $\bullet$. The specific formula for source function, $S\left(y_{2}, \tilde{y}_{2} ; k_{3}^{(s)}, \omega, y_{d}\right)$, that is continuous across the critical level $y_{2}=y_{d}$, where $y_{d}$ is the location where the velocity profile is maximum if the mean flow is symmetric about $y_{d}$, is

$$
S\left(y_{2}, \tilde{y}_{2} ; k_{3}, \omega, y_{d}\right)=\frac{\left|U^{\prime}\left(y_{2}\right) U^{\prime}\left(\tilde{y}_{2}\right)\right|}{\left[U\left(y_{2}\right) U\left(\tilde{y}_{2}\right)\right]} \frac{\left(1+\frac{y_{2}-y_{d}}{y_{d}} b_{0}\right)\left(1+\frac{\tilde{y}_{2}-y_{d}}{y_{d}} b_{0}\right) F_{\perp}\left(y_{d}, y_{d} \mid y_{2}, \tilde{y}_{2}, \omega, k_{3}\right)}{E\left(y_{2} ; k_{3}, \omega, y_{d}\right)\left[E\left(\tilde{y}_{2} ; k_{3}, \omega, y_{d}\right)\right]^{*}}
$$

where

$$
E\left(y_{2} ; k_{3}^{(s)}, \omega, y_{d}\right) \equiv \frac{\left[U\left(y_{d}\right)-U\left(y_{2}\right)\right]}{c^{2}\left(y_{d}\right)}\left(1-\frac{\left|y_{2}-y_{d}\right|}{y_{d}} b_{0}\right)+\frac{\left[\omega^{2} / U^{2}\left(y_{2}\right)+k_{3}^{2}\right]}{\sqrt{\omega^{2} / U^{2}\left(y_{2}\right)+k_{3}^{2}-k_{\infty}^{2}}} \frac{i \pi U^{2}\left(y_{2}\right) b_{0}}{c_{\infty}^{2} U^{\prime \prime} y_{d}},
$$

is an algebraic factor determined through the GAL analysis and * is the complex conjugate. The parameter, $b_{0}$, is a constant that is proportional to the ratio of the even to odd 
symmetry components of the Fourier transform of $\tilde{\omega}_{c}$ (see GAL, Eq. 6.42, and appendix A) and is treated as a parameter in our model. $U^{\prime \prime}\left(y_{d}\right)$ is the second derivative of the mean velocity profile at $y_{d}$.

\subsection{Scattered solution}

The scattered part of the Green's function solution, $g^{(s)}$ in the split formula accounts for the presence of the trailing edge and will satisfy streamwise inhomogeneous boundary and jump conditions on the streamwise-discontinuous surface present in the flow. Imposing appropriate boundary/jump conditions on the plate surface and its downstream extension leads to a Wiener-Hopf problem (Eqs. 6.6-6.8 in GAL) for the (temporal, streamwise and spanwise) Fourier transform of $g^{(s)}$. This can be solved by a routine application of the Wiener-Hopf technique (e.g. Noble [61] and Crighton \& Leppington [62]) and the interested reader is referred to GAL (pp. 550-554 \& app. B) for further details.

\subsection{Acoustic spectrum formula for jet-surface interaction in planar flows}

For a two-dimensional jet with a planar mean flow but with a full three dimensional turbulence spectrum, GAL [21]showed that the acoustic spectrum for jet-surface interaction noise is given by the remarkably simple asymptotic result,

$$
\begin{aligned}
I_{\omega}(\boldsymbol{x}) \equiv \frac{1}{2 \pi} \int_{-\infty}^{\infty} e^{i \omega \tau} \overline{p^{s}(\boldsymbol{x}, t) p^{s}(\boldsymbol{x}, t+\tau)} d \tau \\
\quad \approx\left(\frac{k_{\infty}}{4 \pi|\boldsymbol{x}|}\right)^{2} \int_{0}^{\infty} \int_{0}^{\infty} D\left(M\left(y_{2}\right), M\left(\tilde{y}_{2}\right) ; \theta, \psi\right) S\left(y_{2}, \tilde{y}_{2} ; k_{3}^{(s)}, \omega, y_{d}\right) d y_{2} d \tilde{y}_{2}
\end{aligned}
$$

in which the integrand can be interpreted as the product of a source function $S\left(y_{2}, \tilde{y}_{2} ; k_{3}^{(s)}, \omega, y_{d}\right)$, which is the auto-covariance of $\tilde{\omega}_{c}$, with a non-uniform directivity factor, $D\left(M\left(y_{2}\right), M\left(\tilde{y}_{2}\right) ; \theta, \psi\right)$. The former can be approximately related to upstream turbulence statistics, via. Eq. (10), and the latter encapsulates the mean flow interaction effect and was derived using the Wiener-Hopf techniqe in GAL using a (low frequency) asymptotic solution to Rayleigh's equation when the far-field wavenumber is asymptotically small, i.e. $k_{\infty} \ll O(1) . M\left(y_{2}\right)=U\left(y_{2}\right) / c_{\infty}$ denotes the local acoustic Mach number at the position $y_{2}$ and the spanwise wavenumber $k_{3}^{(s)}=k_{\infty} \sin \theta \cos \psi$ is found by applying the method of stationary phase for the inverse Fourier integral in $k_{3}$ ([24], p. 553) when $|\boldsymbol{x}| \rightarrow \infty$ where $\theta$ is the polar observation angle measured relative to the jet center line and $\psi$ is the azimuthal angle in the cross-stream plane. The above result bears some resemblance to acoustic analogy approaches in which the acoustic spectrum can be formally interpreted as the product of a propagator and a turbulence source term. However, as opposed to these 
approaches, here, only a single component of turbulence correlation function enters Eq. (10) at pre-determined transverse locations, $\boldsymbol{x}_{T}$, as required by the inversion of the spectrum of Eq. (6) ([24], sec. 4).

Note that the assumption of a semi-infinite/doubly infinite streamwise/spanwise plate, which is required to perform the Wiener-Hopf analysis analytically to obtain Eq. (12), has been assessed by Cavalieri et al. [63] and Bridges et al. [1]. Both of whom found a slight amplification (Fig. 9 in [1] \& p. 11 \& ff.) and departure in dipole symmetry for the azimuthal directivity (Fig. 14 in [63]) when finite-dimension plates were considered. The latter was found to arise from, among other things, the interaction between the trailing edge noise source and that generated by the lateral plate edges [63]. Therefore, the level of asymmetry/lobe amplification in the directivity pattern will depend on the dimensions of the plate. But the NASA data, to which we compare results from our model, was designed such that streamwise/spanwise plate dimensions $\left(L_{1}, L_{3}\right) \gg D_{J}$ in which case any asymmetry/amplification effect due to lateral plate dimensions should be negligible.

2.7 Directional structure of GAL asymptotic formula, Eq.(12)

The directivity factor, which is given by

$$
D\left(M\left(y_{2}\right), M\left(\tilde{y}_{2}\right) ; \theta, \psi\right)=\frac{\left[M\left(y_{2}\right) M\left(\tilde{y}_{2}\right)\right]^{3 / 2}(\beta-\cos \theta)}{\left[1-M\left(y_{2}\right) \cos \theta\right]\left[1-M\left(\tilde{y}_{2}\right) \cos \theta\right] \sqrt{\left[1-\beta M\left(y_{2}\right)\right]\left[1-\beta M\left(\tilde{y}_{2}\right)\right]}},
$$

is proportional to Goldstein's ([23], Eq. 3.23) result $\sin ^{2}(\theta / 2) /\left(1-M\left(y_{d}\right) \cos \theta\right)^{2}$ at the point where the velocity profile is maximum $y_{2}=\tilde{y}_{2}=y_{d}$ since $\beta \equiv\left(1-\sin ^{2} \theta \cos ^{2} \psi\right)^{1 / 2}$ is unity at $\psi= \pm \pi / 2$ in the plane perpendicular to the plate. This factor, of course, also generalizes Ffowcs-Williams \& Hawking's [25] result (1970, Eq. 11), which possesses a symmetric cardioid directivity shape with polar angle. That is, of the $1-\cos \theta=\sin ^{2}(\theta / 2)$ form in the plane perpendicular to the plate where $\theta$ is the polar observation angle with respect to the streamwise direction. Note that our result must be transformed to theirs via: $\sin ^{2}(\theta / 2)=\cos ^{2}(\pi-\theta / 2)$ owing to their co-ordinate system shown (see p. 620 of [23]). The GAL result (13) extends the Ffowcs-Williams Hall theory to non-uniform mean flows.

Commensurate with this shape for polar directivity, the azimuthal directivity was found to have to be characterisitic of a vertical lemniscate loop (Howe [64], fig. 2.7.1) symmetric about the horizontal axis which is usual distribution for an acoustic dipole [65]. This shape was also found in computational study by Wolf \& Lele ([66] Fig. $13 \& 14$ ) and is shown below in our Fig. 1. The figure show the polar directivity at fixed $\psi= \pm \pi / 2$ and the azimuthal character of $D\left(M\left(y_{2}\right), M\left(\tilde{y}_{2}\right) ; \theta, \psi\right)$ at various polar angles where $M\left(y_{2}\right)=M\left(\tilde{y}_{2}\right)=M a$. Note that the arrows indicate increasing jet acoustic Mach number. The results show that 
$D(\theta, \psi, M a)$ peaks at a polar angle of $\theta=90^{\circ}$ at $M a=0.5$. The reason for this is that the the cardioid shaped curve, Eq. (13), possesses a cusp at $\theta \rightarrow 0$ and has the peak directivity (at azimuthal angle of $\psi= \pm \pi / 2$ radians) at $\cos \theta=2-1 / M a$ where $M a$ is the peak Mach number of the velocity profile. It is, therefore, only for low Mach numbers in the forward measurement arc that the directivity peaks near $\theta=90^{\circ}$. On the other hand, the polar directivity at $\theta=30^{\circ}$ is about 2.7 times greater than at $\theta=90^{\circ}$ for $M a=0.9$. This corresponds to, approximately, a $4 \mathrm{~dB}$ increase if distributed everywhere in the flow, but the large difference only occurs at the peak velocity location (i.e. at one point in the acoustic spectrum formula (12)). 

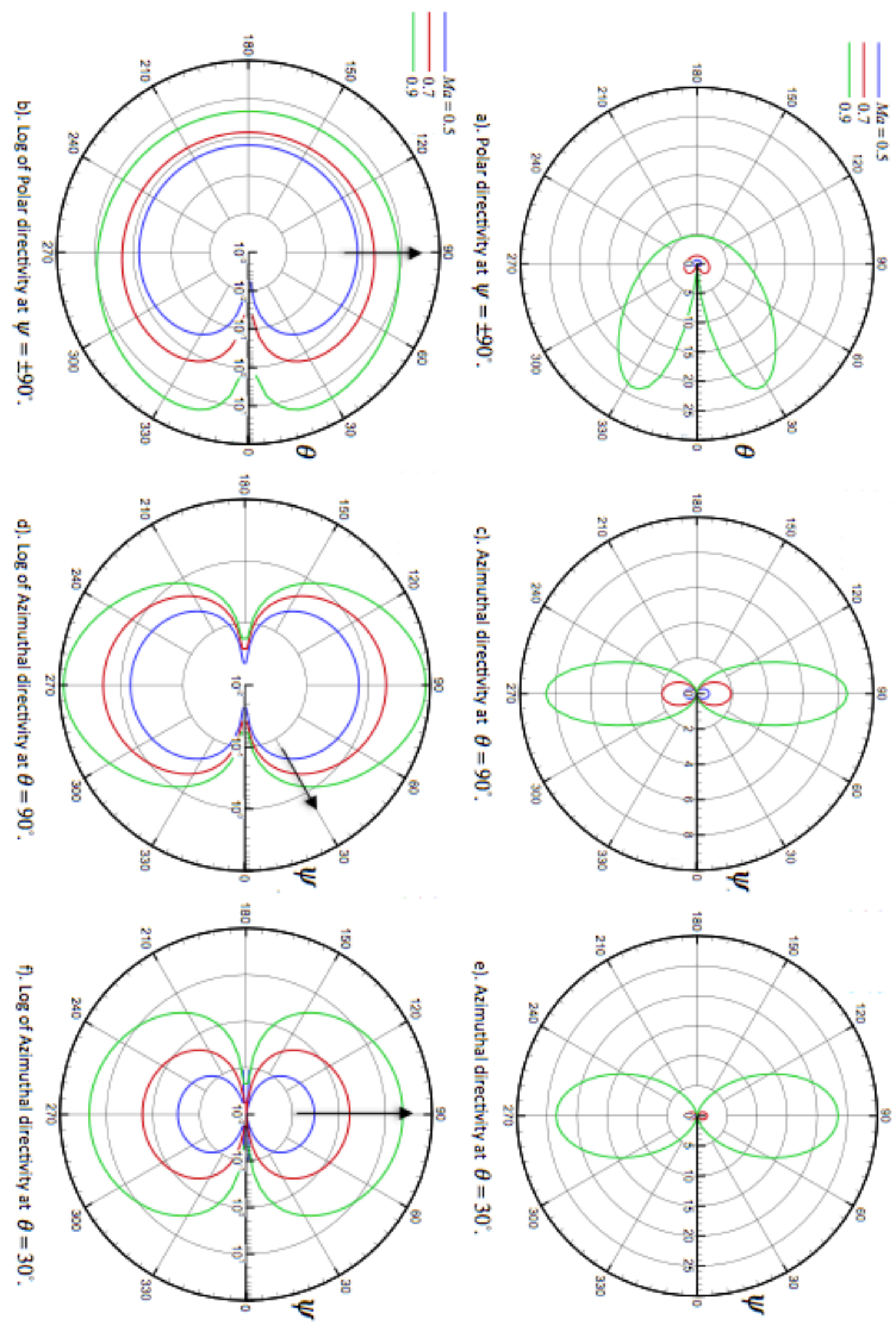

Fig. 1. Properties of the Directivity factor, Eq. (13). The arrow is in direction of increasing acoustic Mach number, $M a$. 


\section{TURBULENCE MODELLING}

\subsection{Modeling de-correlation effects in $\left\langle\rho v_{\perp}^{\prime(0)}(x, t) \rho v_{\perp}^{\prime(0)}\left(\tilde{x}_{1}, \tilde{x}_{2}, x_{3}+\eta_{3}, t+\tau\right)\right\rangle$}

The jet-surface interaction noise model Eq. (12) was constructed for a two-dimensional jet with planar mean flow. Consistent with this approximation, we suppose that the turbulence is spanwise homogeneous. The statistical quantity,

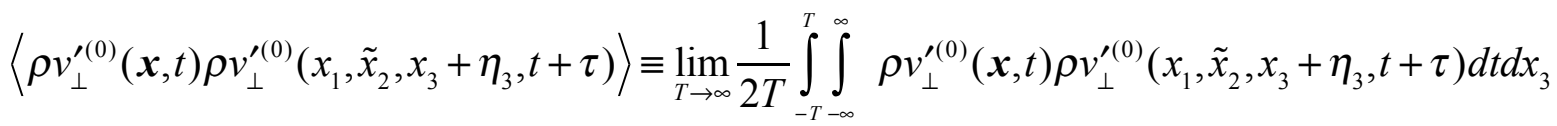

that enters the integrand of Eq. (9) is both experimentally determinable and has a well established database in free round jets [67]. In this section, we construct an improved model compared with GAL for this function and derive an explicit formula for the acoustic spectrum. Differently from [24], however, the model we use for correlation function in Eq. (14) now allows for a negative dip in space separation and time delay, which we show has a direct impact on the jet surface interaction noise and, in principle, could provide a means to reduce it (we discuss this point further in Sec. 7). We, therefore, use the function based on the model in [38]):

$$
\begin{aligned}
& \left\langle\rho v_{\perp}^{\prime(0)}\left(x_{1}, y_{d}, x_{3}, t\right) \rho v_{\perp}^{(0)}\left(\tilde{x}_{1}, y_{d}, x_{3}+\eta_{3}, t+\tau\right)\right\rangle= \\
& L_{3} \Psi\left(\bar{x}_{1}\right)\left[a_{0}+a_{1} \tau \frac{\partial}{\partial \tau}+a_{2} \eta_{1} \frac{\partial}{\partial \eta_{1}}+\ldots\right] e^{-X\left(\eta_{1},|\eta| \tau ; \alpha\right)}
\end{aligned}
$$

where coefficients, $a_{n}=\left(a_{0}, a_{1}, a_{2}\right)$, are empirical parameters and the decay function multiplying the square brackets is defined by exponent,

$$
X\left(\eta_{1},|\eta|, \tau ; \alpha\right)=\sqrt{\left(\eta_{1} / l_{1}\right)^{2}+\left(\eta_{1}-U_{c} \tau\right)^{2} / l_{0}^{2}+\left(\eta_{3} / l_{3}\right)^{2}+\alpha^{2}}
$$

where $\alpha$ is a small positive number, $0 \leq \alpha<1$, that governs the sharpness of the autocorrelation of Eq. (15) at zero time delay and zero spatial separation and the streamwise and spanwise separations are $\eta_{1} \equiv \tilde{x}_{1}-x_{1}$ and $\eta_{3}$ respectively. The scales $l_{1}$ and $l_{3}$ are turbulence length scales in the streamwise and spanwise directions, respectively; $l_{0}$, on the other hand, measures the moving frame streamwise length scale of the turbulence.

Following usual practise (see [38]) we allow $\Psi$ to decay in the streamwise direction in order to insure convergence of the subsequent Fourier transform integrals, we replace $\Psi\left(\bar{x}_{1}\right)$ by i.e. $\Psi_{0} e^{\alpha-\left(\bar{x}_{1} / L_{1}\right)^{2}}$. The amplitude, $\Psi\left(\bar{x}_{1}\right)$, depends on the symmetric location $\bar{x}_{1} \equiv\left(x_{1}+\tilde{x}_{1}\right) / 2$, since this quantity is expected to be independent of the streamwise 
coordinate for the nearly parallel flow being considered. $\Psi_{0}$ is expected to scale with the transverse component of the mean square turbulence momentum flux $\overline{\left(\rho v_{\perp}^{\prime}\right)^{2}},\left(L_{1}, L_{3}\right)$ are geometric spatial scales: $L_{3}$ being a measure of the spanwise extent of the turbulence enters as pre-factor on the right hand side of (15) and the streamwise scale, $L_{1}$, which is taken to be large in order to insure that Eq. (15) is relatively independent of $\bar{x}_{1}$. This is consistent with our representation of the upstream boundary condition described in section 2 .

As we show in Fig. 2, allowing the coefficient $a_{1}>0$ in Eq. (15) gives a negative (or, decorrelation) region for the auto-correlation $\left(\eta_{3}=0\right)$ function of Eq. (15) which did not appear in the model used in GAL (Eq. 6.46). As mentioned in the Introduction, the presence of this negative region in the second-order transverse velocity correlations has been measured in various turbulence experiments in the literature (e.g., Townsend [68]; p. 134 \& Fig. 4.16 of [39] and p. 252f. of [40]). Unfortunately, experimental data for the turbulence correlation function (14) in a planar jet with a trailing edge in the flow is not available for comparison with our model. However, qualitative similarity does exist between our model and Gutmark \& Wygnanski [69] who indicate (on p.480 \& Fig. 17) that "the negative dip in the lateral correlation (defined on p.480) in the two-dimensional jet is stronger than that in the axisymmetric jet.." . The jet in their experiments issued from a nozzle with very large aspect ratio (almost 38:1), but a very low speed flow $(35 \mathrm{~m} / \mathrm{s}$ ) at room temperature. Indeed, Fig. 19 in [69] shows that the normalized level of de-correlation in the lateral correlation function can be as large as -0.5 at particular locations in the jet.

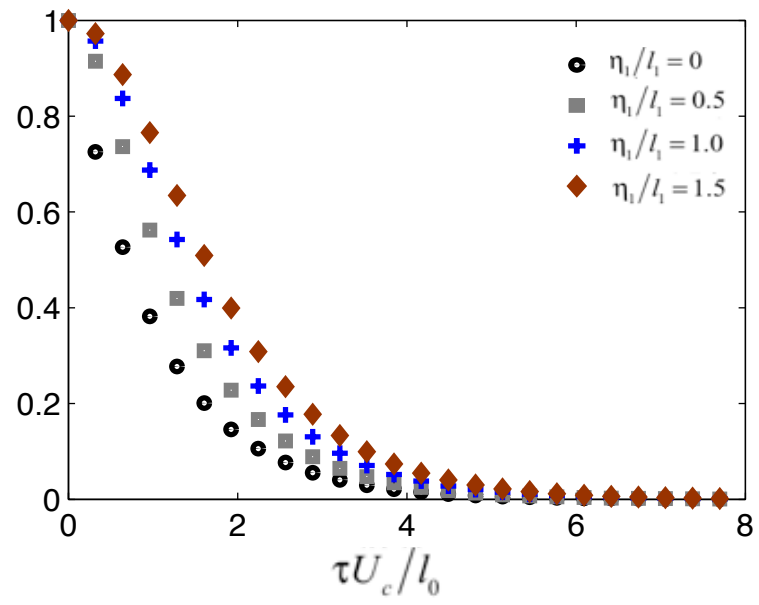

(a). GAL model, $a_{1}=a_{2}=0$,

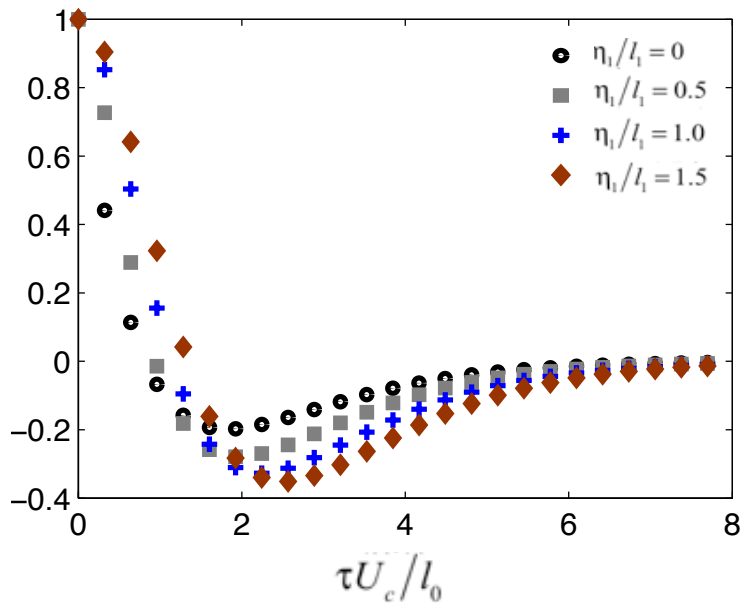

(b). Latest, $\left(a_{1}, a_{2}\right) \neq 0$.

Fig. 2. Auto-correlation of upstream turbulence using model Eqs. (15) \& (16) with $\left(\eta_{3}=0\right),(\alpha=0)$ \& $l_{r}=l_{1} / l_{0}$ given by caption in Fig. 5. 


\subsection{Jet-surface interaction acoustic spectrum formula with a finite de-correlation region}

Substituting the source model function Eq. (15) in Eqs. (9)-(11) for $S\left(y_{2}, \tilde{y}_{2}, k_{3}^{(s)}, \omega, y_{d}\right)$ and inserting this result in Eq. (12), we obtain the final formula for the acoustic spectrum used in this paper

$$
I_{\omega}(\boldsymbol{x})=\left(\frac{1}{4 \pi|\boldsymbol{x}|}\right)^{2}\left(\frac{1}{\pi^{2}}\right) \frac{l_{0} l_{1} l_{3}}{U_{c}}\left(\frac{L_{3} \Psi_{0} k_{\infty}}{c_{\infty}}\right) I_{0}\left(M a, \theta, \psi ; y_{d}\right)
$$

The integral $I_{0}\left(M a, \theta, \psi ; y_{d}\right)$ is defined by

$$
I_{0}\left(M a, \theta, \psi ; y_{d}\right)=4 \int_{0}^{M a} c_{\infty} D\left(M\left(y_{2}\right) ; \theta, \psi\right) \frac{\tilde{\Pi}\left(\omega / U\left(y_{2}\right), \omega, k_{3}^{(s)}\right)}{\left|E\left(y_{2} ; k_{3}^{(s)}, \omega, y_{d}\right)\right|^{2}} d M\left(y_{2}\right)
$$

where $D\left(M\left(y_{2}\right) ; \theta, \psi\right)$ is equal to directivity factor (13) at $M\left(y_{2}\right)=M\left(\tilde{y}_{2}\right)$. This result now depends parametrically on the transverse location of the peak of the jet velocity relative to the trailing edge, $y_{d}$. Also in Eq. (18), $M a \equiv U\left(y_{d}\right) / c_{\infty}$ denotes the maximum acoustic Mach number at the axial location of the trailing edge. Some details of this derivation using the new model function are given in appendix A for completeness. The relative location of the nozzle exit and the plate trailing edge is illustrated by the schematic in Fig. 3.

This result, although formally similar to GAL (their Eqs. 6.50 \& 6.53), has very different spectral behaviour owing to the 'de-correlation-dependent' coefficient $\tilde{\Pi}\left(\omega / U\left(y_{2}\right), \omega, k_{3}^{(s)}\right)$, which in GAL is equal to $a_{0} /\left(\left(\omega l_{0} / U_{c}\right)^{2}+\chi\right)^{2}$, but in our case (see derivation in App. A \& Eq. (A.19)) is found to be:

$$
\begin{aligned}
& \tilde{\Pi}\left(k_{1}, \omega, k_{3}\right)= \\
& \frac{1}{\left(\left(\omega l_{0} / U_{c}\right)^{2}+\chi\right)^{2}}\left[\left(a_{0}-a_{1}-a_{2}\right)+\frac{4}{\left(\left(\omega l_{0} / U_{c}\right)^{2}+\chi\right)}\left[a_{1}\left(\frac{\omega l_{0}}{U_{c}}\right)^{2}-\left(a_{1} \frac{\omega}{U_{c}}-a_{2} k_{1}\right)\left(k_{1}-\omega / U_{c}\right) l_{1}^{2}\right]\right]
\end{aligned}
$$

where

$$
\chi=\chi\left(k_{1}, k_{3}\right)=\left[\left(k_{3} l_{3}\right)^{2}+\left(k_{1}-\omega / U_{c}\right)^{2} l_{1}^{2}+1\right] .
$$

and $\alpha=0$ in Eqs. (15) \& (16). The impact of these spectral differences on the edge noise spectrum is discussed in the next section. 


\section{ACOUSTIC SPECTRUM WITH A PURELY DIPOLE-LIKE FREQUENCY SCALING}

The predictions in GAL were compared against the jet-surface interaction experiments performed at NASA Glenn [3].

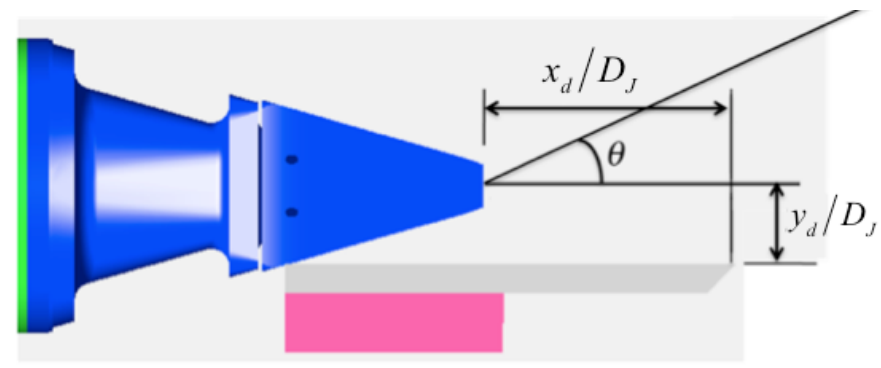

Fig. 3 - Nozzle/plate configuration (courtesy Dr. James E. Bridges, NASA Glenn Center)

However it is clear from Fig. 6 in GAL, that for Strouhal numbers less than 0.15 , the purely positive auto-correlation model (i.e. setting $a_{1}=a_{2}=0$ in Eq. (15)) they used over predicts the low-frequency roll-off for jet-surface interaction noise by as much as $10 \mathrm{~dB}$ (see Fig. $6 \mathrm{~d}$ in $\mathrm{GAL})$.

We can easily prove why this occurs by estimating integral $I_{0}\left(M a, \theta, \psi ; y_{d}\right)$. It is clear from Eq. (18) that as $\omega \rightarrow 0$, the dominant contribution to the integral comes from the vicinity of critical level $y_{2}=O\left(y_{d}\right)$. In App. B, we estimate Eq. (18) under this limit at $\psi=-90^{\circ}$ (in which case, $\beta=1$ and $k_{3}^{(s)}=0$ ). Substituting Eq. (B.6) into acoustic spectrum formula Eq. (17) shows that the latter possesses the following algebraic decay rates,

$$
I_{\omega}(\boldsymbol{x})=\left\{\begin{array}{c}
O(1), \text { for } a_{1}=a_{2}=0 \text { (GAL.) } \\
O\left(\omega^{2}\right), \text { for } a_{0} \sim a_{1} \text { (Present model, Eq. 16.) }
\end{array}\right.
$$

as $\omega \rightarrow 0$.

These results show that the acoustic spectrum is now directly dependent on how fast the turbulence de-correlates in Eq. (15). In its absence (i.e. $a_{1}=a_{2}=0$ ) Eq. (21) shows that the acoustic spectrum does not possess a low-frequency roll-off as such and tends to $O(1)$ (i.e. a constant, see Eq. (6.52) in GAL). A finite de-correlation region, however, increases the low frequency 'roll-off' in the prediction of Eq. (17) and causes it to exhibit a pre-factor of the order of $O\left(\omega^{2}\right)$, which is a purely dipole-like freqeuency scaling and appears to be more consistent with the experimental data at Strouhal numbers greater than peak frequency and closer to the data for frequencies below the peak. 


\subsection{Sensitivity of model to parameters $\left(a_{1}, a_{2}\right)$}

In Fig. 4 we show how sensitive the magnitude of the negative dip in Eq. (15) is to parameters $\left(a_{1}, a_{2}\right)$ (corresponding to a length scale ratio, $l_{r}=l_{1} / l_{0}$, given in the caption to Fig. 5) and in Fig. 5 we show how this impacts the acoustic spectrum. We compare our predictions against experimental data measured at the shielded location with the microphone array below plate [3]. Note that this location is chosen to avoid any spurious acoustic contributions from reflections when the microphone is above the plate-see [3]\&[20]. Moreover, we use the same Gaussian mean velocity profile (Eq. 6.55) used in GAL to define the jet mean velocity; the decay parameters of the profile were chosen to match experimental data reported in [19] for the large aspect ratio (AR) rectangular nozzle of 8:1 (AR8). Fig. 4 indicates that when $a_{1} \sim a_{0}$ a significant negative dip appears in the correlation function. We have not shown the sensitivity of Eq. (15) to $a_{2}$ in Fig. 4 , since our numerical experiments indicated it had a small impact. The reason why this is the case is clear if we carry out the derivatives in (15) explicitly; i.e. for $(\alpha=0)$,

$$
\begin{aligned}
& \left\langle\rho v_{\perp}^{\prime(0)}\left(x_{1}, y_{d}, x_{3}, t\right) \rho v_{\perp}^{(0)}\left(\tilde{x}_{1}, y_{d}, x_{3}+\eta_{3}, t+\tau\right)\right\rangle= \\
& L_{3} \Psi\left(\bar{x}_{1}\right)\left[a_{0}-a_{1} \frac{\tilde{\tau}\left(\tilde{\tau}-\tilde{\eta}_{1} l_{r}\right)}{X}-a_{2} \frac{\tilde{\eta}_{1}\left(\tilde{\eta}_{1}+l_{r}\left(\tilde{\eta}_{1} l_{r}-\tilde{\tau}\right)\right.}{X}+\ldots\right] e^{-X\left(\eta_{1},|\eta|, \tau ; 0\right)} .
\end{aligned}
$$

$\tilde{\eta}_{1}=\eta_{1} / l_{1}$ and $\tilde{\tau}=U_{c} \tau / l_{0}$ are non-dimensional spatial separation and time delay respectively and $l_{r}=l_{1} / l_{0}$ is ratio of length scales. Since $l_{r} \ll 1$ for the time-stationary turbulence we are concerned with, the second term (22) will be smaller in magnitude even when $a_{2} \sim a_{0}$.
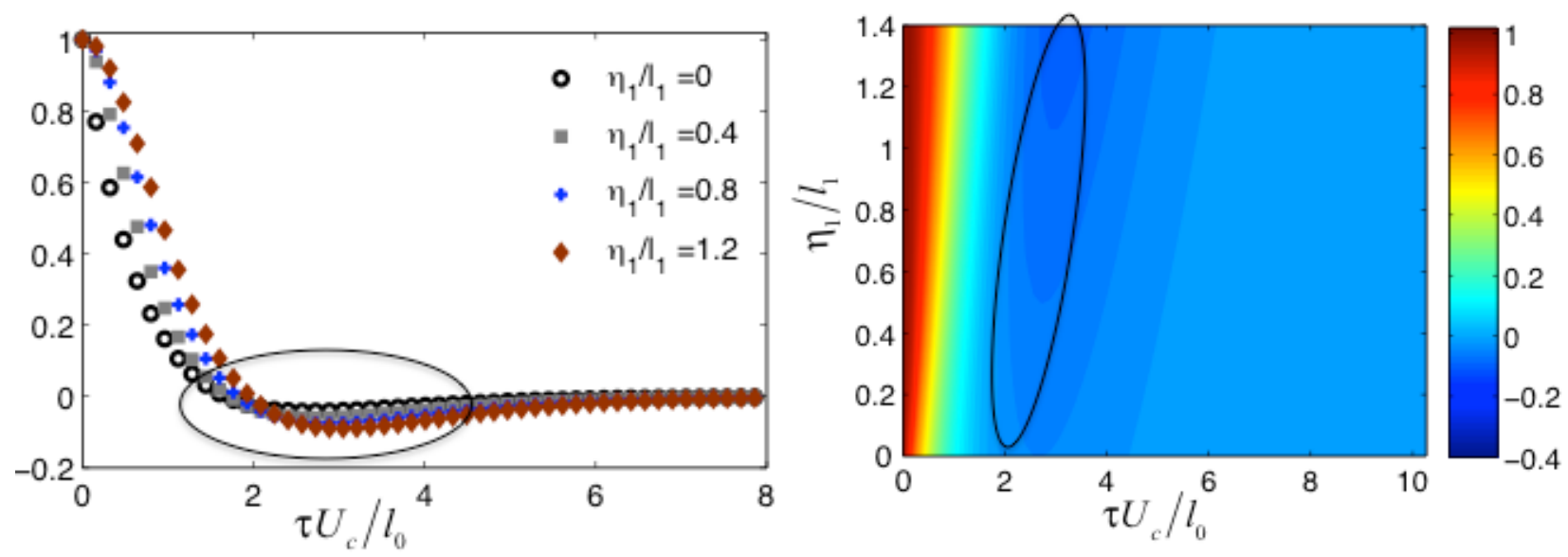

(a). Turbulence model, Eq. (15) for parameter values $\left(a_{0}, a_{1}, a_{2}\right)=(1,0.6,0)$. 

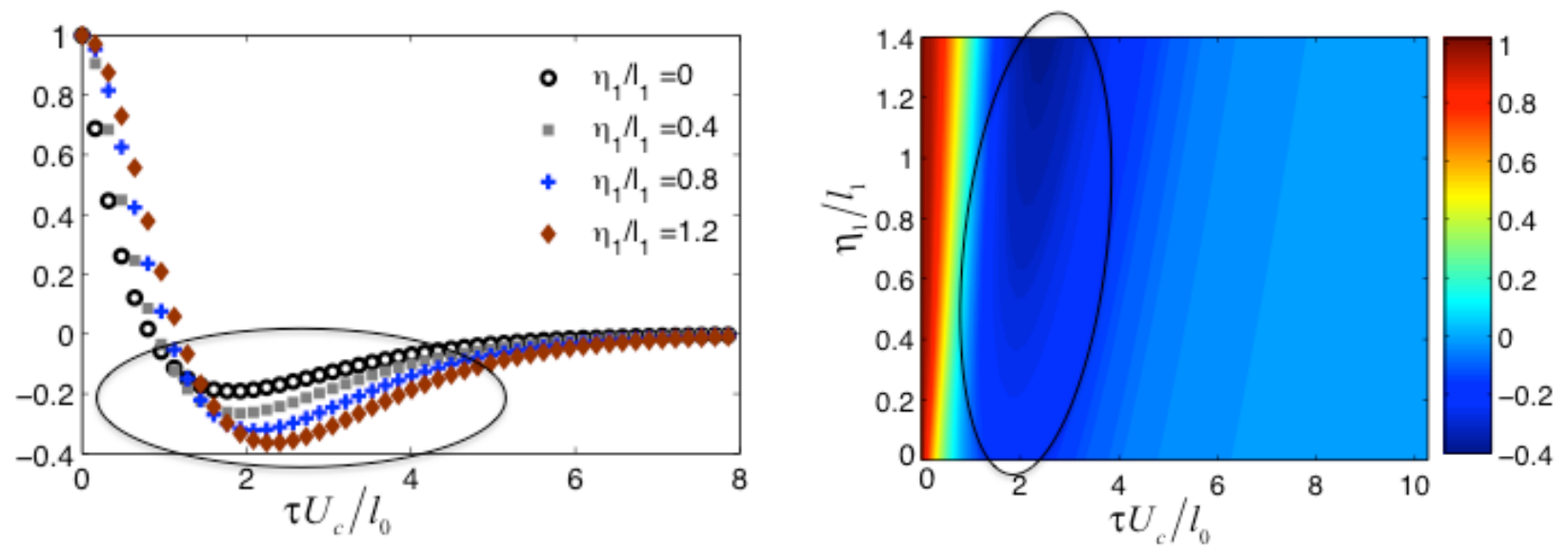

(b). Turbulence model, Eq. (15) for parameter values $\left(a_{0}, a_{1}, a_{2}\right)=(1,1.2,0)$.

Fig. 4 - Normalized space-time variation of Eq. (15) and its spatial distribution when $a_{1} \sim a_{0}\left(l_{r}=l_{1} / l_{0}\right.$ as in Fig. 5).

The sensitivity of the acoustic predictions in Fig. 5 show that, for $a_{1} \sim a_{0}$ (with $a_{2}=0$ ) in Eq. (15), the low frequency roll-off more closely follows the data compared to the GAL prediction. In Fig. 5, and all the following comparisons, data for the component of the noise associated with the trailing edge is obtained from the total measured noise by subtracting the noise measured in the corresponding free jet (i.e. in the absence of a plate). Consistent with Fig. 4, our numerical experiments show that moderate values of $a_{2}$ do not change the algebraic decay of the predictions (i.e. $I_{\omega}(\boldsymbol{x}) \sim O\left(\omega^{2}\right)$ remains as long as $\left.a_{1} \sim a_{0}\right)$. This can be explained by the fact when $k_{1} \rightarrow \omega / U\left(y_{2}\right)$ the second term in square brackets in Eq. (19), makes a relatively small contribution to $\tilde{\Pi}\left(\omega / U\left(y_{2}\right), \omega, k_{3}^{(s)}\right)$ for high acoustic Mach numbers. In Fig. $5 d$ we assess this numerically by considering the ad-hoc approximation to Eq. (19),

$$
\tilde{\Pi}\left(\omega / U\left(y_{2}\right), \omega, k_{3}^{(s)}\right) \approx \frac{1}{\left(\left(\omega l_{0} / U_{c}\right)^{2}+\chi\right)^{2}}\left[\left(a_{0}-a_{1}-a_{2}\right)+\frac{4 a_{1}}{\left(\left(\omega l_{0} / U_{c}\right)^{2}+\chi\right)}\left(\frac{\omega l_{0}}{U_{c}}\right)^{2}\right]
$$

that neglects this term with $\left(1 / U-1 / U_{c}\right)$ factor. Our calculations show that the predictions remain basically unaltered to the case when the full Eq. (19) is used in the acoustic spectrum formula, (17); i.e. the green dot-dashed curve in $5 d$ is more-or-less identical to solid curve in Fig. 5b. The parameter $a_{2}$, therefore, only appears to effect the amplitude of $I_{\omega}(\boldsymbol{x})$ through the $\left(a_{0}-a_{1}-a_{2}\right)$ factor in (19). 


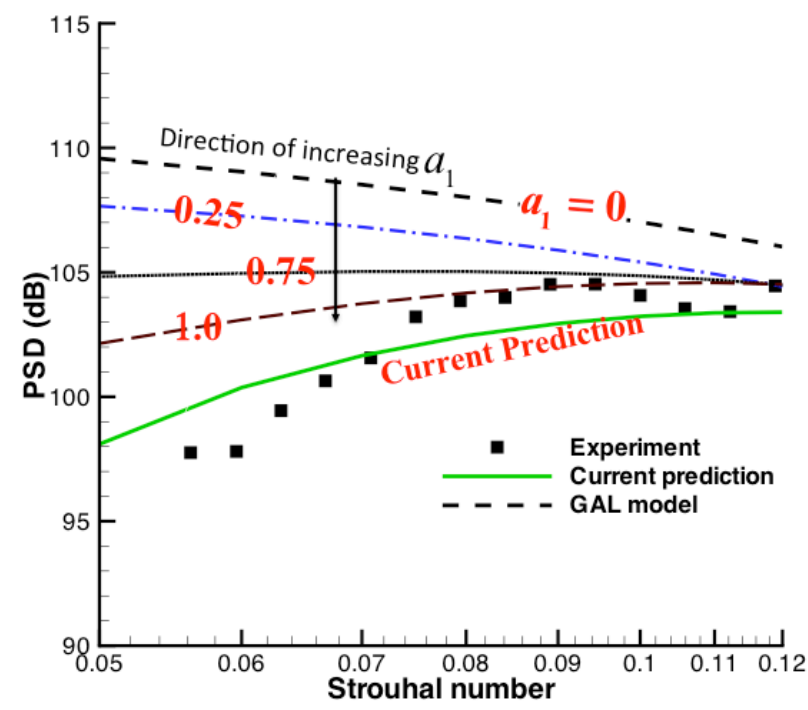

(a). Parametric increase in $a_{1}$ with $\left(a_{0}=1 ; a_{2}=0\right)$.

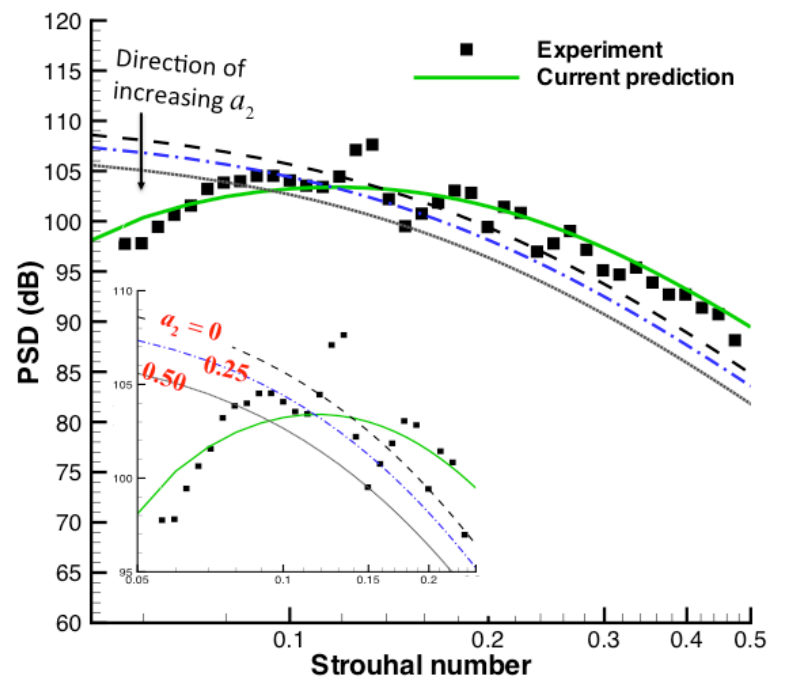

(c). Parametric increase in $\left|a_{2}\right|$ with $\left(a_{0}=1 ; a_{1}=0\right)$.

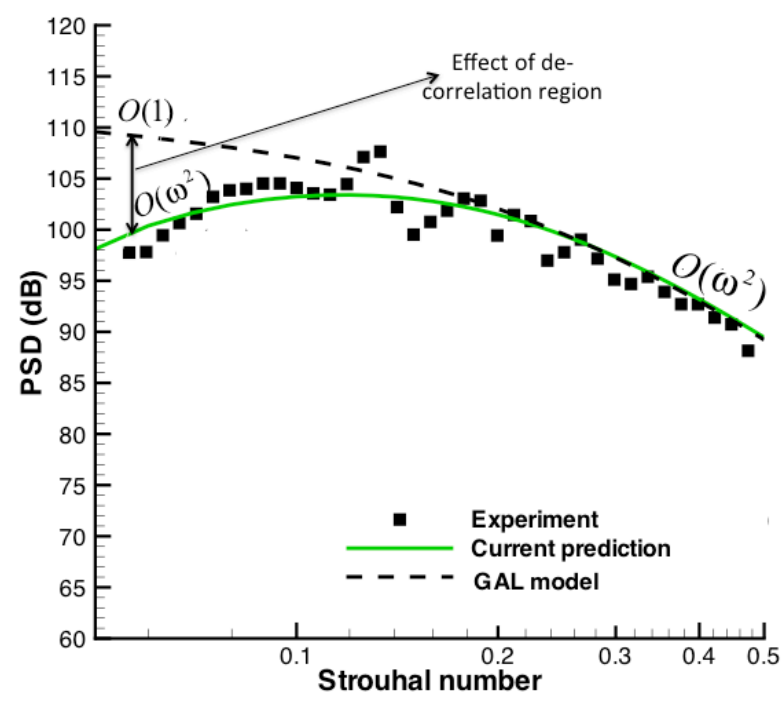

(b). Eq. (17) : using turbulence model, Eq. (15).

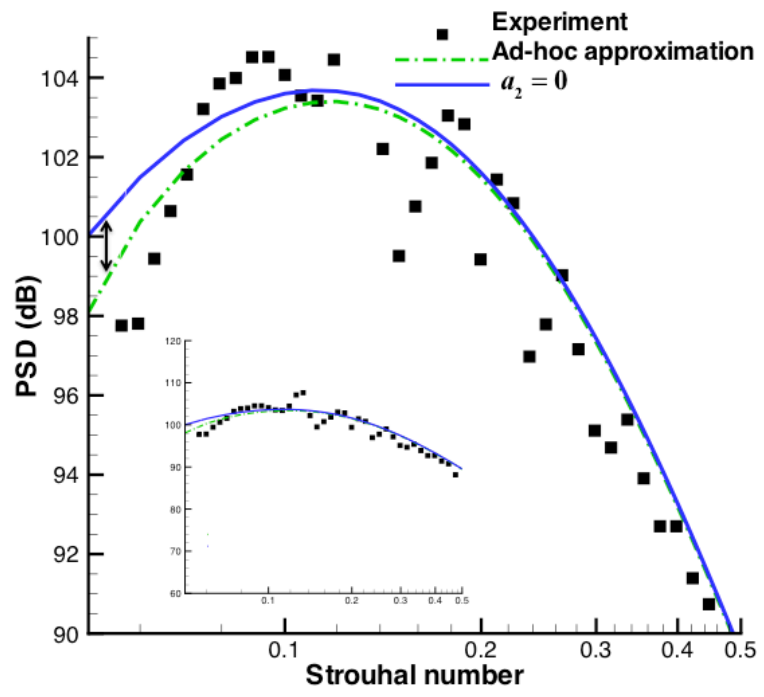

(d). Eq. (17) : using turbulence model, Eq.

(15)

Fig. 5- Power Spectral Density (PSD) of the far-field pressure fluctuations at 100 equivalent diameters from nozzle exit (lossless in $\mathrm{dB}$ scale referenced to $20 \mu \mathrm{Pa}$ ) as a function of Strouhal number for $M a=0.9$. Plate trailing edge at $y_{d} / D_{J}=1.1, x_{d} / D_{J}=5.7, D_{J}=2.12^{\prime \prime}, \psi=-90^{\circ}$ and $\theta=90^{\circ}$. Source model constants for GAL model are the same as their Fig. 4 and for current predictions are

$$
\begin{gathered}
\Psi_{0}=0.04\left(\rho_{\infty} U_{d}\right)^{2} ;\left(l_{0}, l_{1}, l_{3}\right) / D_{J}=(0.53,0.01,0.01) ; L_{3} / D_{J}=20, U_{c}=0.68 U_{d} ; b_{0}=0.52 \text { and } \\
\left(a_{0}, a_{1}, a_{2}\right)=(0.72,0.88,-0.05) \text {. Experimental data obtained from [3]. }
\end{gathered}
$$


In Fig. 6 we show that the acoustic spectrum Eq. (17) possesses a dipole-like vertical lemniscate azimuthal structure, which is similar to the directivity of $D\left(M\left(y_{2}\right) ; \theta, \psi\right)$ alone in Fig. 1, but now it appears to be much flatter. Here, we consider a polar angle of $90^{\circ}$ (since the Bridges/Brown measurements indicate that for the forward arc the jet-surface interaction noise is greatest here) and a peak Strouhal number, which from Fig. 5b, occurs at about $S t=0.12$ (Fig. 6a) and where the upper limit of amplification is near $S t=0.5$ (Fig. 5b).

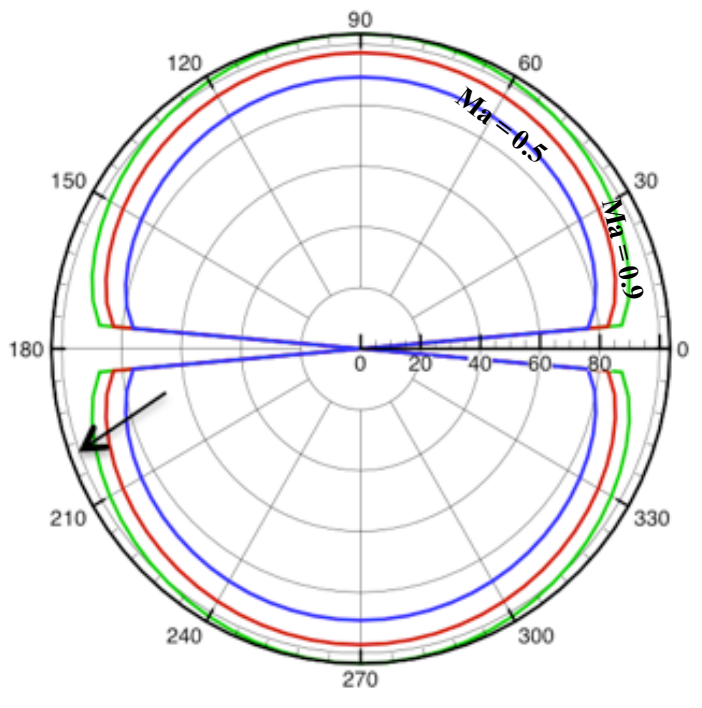

(a). Peak Strouhal number, $S t=0.12$, in Fig. $5 b$.

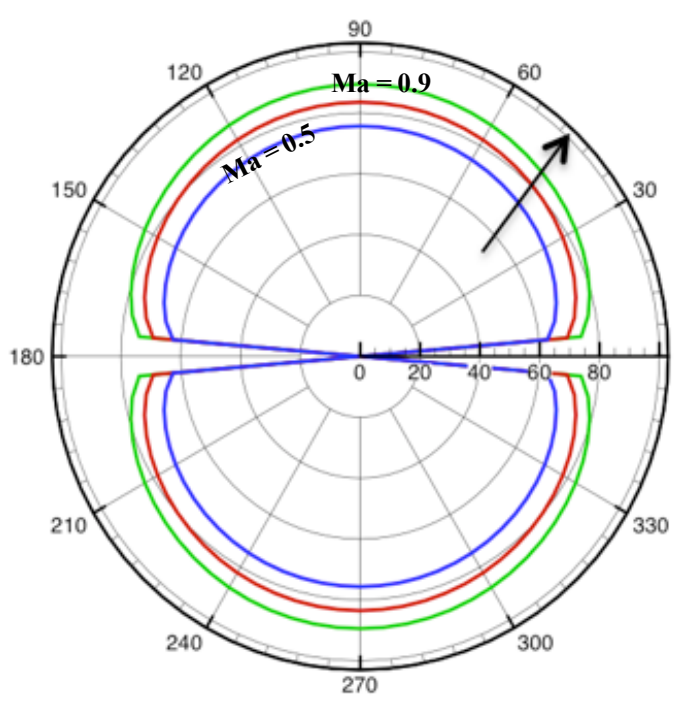

(b). Upper limit, $S t=0.5$, in Fig. $5 \mathrm{~b}$

Fig. 6 PSD (dB) of $\psi$-directivity of RDT prediction Eq. (17) at peak Strouhal number (St) and upper limit as shown in

Fig. 5 for $M\left(y_{2}\right)=M a=(0.5,0.7,0.9)$ (colour coding same as Fig. 1). Source model constants for RDT prediction is same as Fig. 5. Arrow in direction of increasing Ma.

It is clear in Figs. (7) - (9), where the results were computed using the same parameters as in Fig. (5), (see figure caption for values) and the calibration was performed at $M a=0.9$, that the predictions provide a first estimate of the jet-surface interaction noise over a wide range of nozzle aspect ratios ( $2: 1$ to $8: 1$, which we refer to as $A R 2-A R 8$ respectively), jet exit Mach numbers and observation angles. The predictions are closer to the data for the highest Mach numbers and at the higher end of the Strouhal number range (i.e. St $\sim 0.4-0.5$ ) over which amplification occurs. The agreement is closer to the data than GAL's results for the large aspect ratio $A R 8$, since $I_{\omega}(\boldsymbol{x})=O\left(\omega^{2}\right)$ now acts as a pure dipole acoustic source over the frequency range of amplification, however, it is clear from Figs. (7) - (9), that at Strouhal numbers lower than the peak frequency the data appears to decay faster than $O\left(\omega^{2}\right)$. This is more evident for the $M a=0.9$ jet in Fig. 7. Nonetheless, the predicted noise lies within $5 \mathrm{~dB}$ across the range of Mach number and polar observation angle. The data shows greater variation with $A R$ at the lowest frequencies (in the roll-off region) than at $S t \sim 0.4-0.5$ ) 
and, therefore, the RDT model (which striclty does not account for non-planar flow effects) provides much better agreement with data for Strouhal numbers greater than the peak frequency (e.g. Figs. 7c \& 8c). The difference between prediction and data is much larger at $M a=0.5$, which is not surprising given that parameters in the acoustic spectrum formula (Eqs. (17) \& (18)) were tuned against the far-field noise data at the $M a=0.9 \& \theta=90^{\circ}$ measurement location.

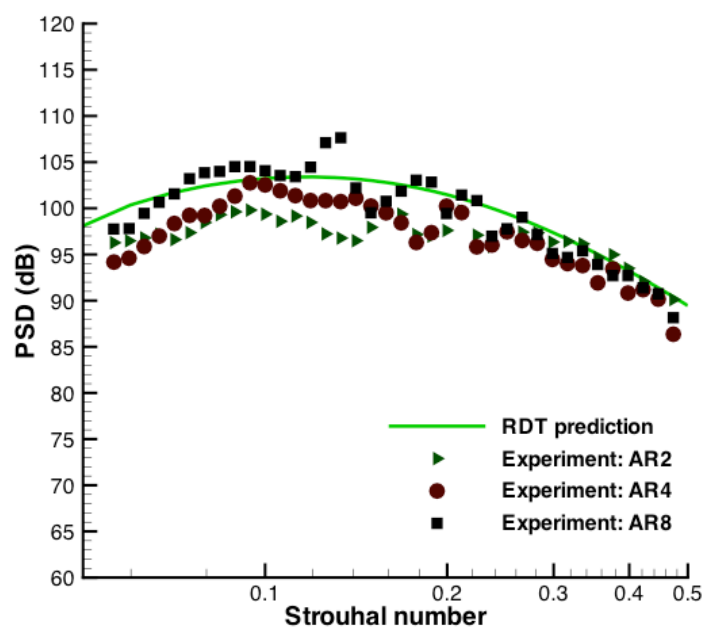

(a). $\theta=90^{\circ}$

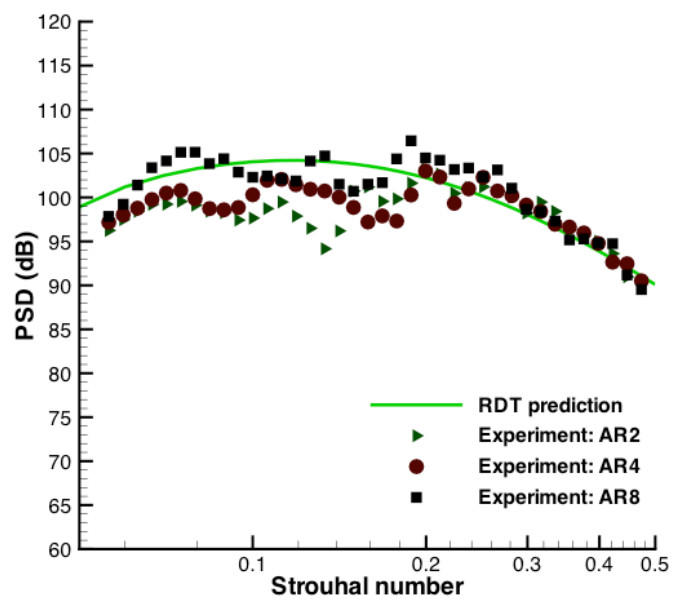

(c). $\theta=60^{\circ}$

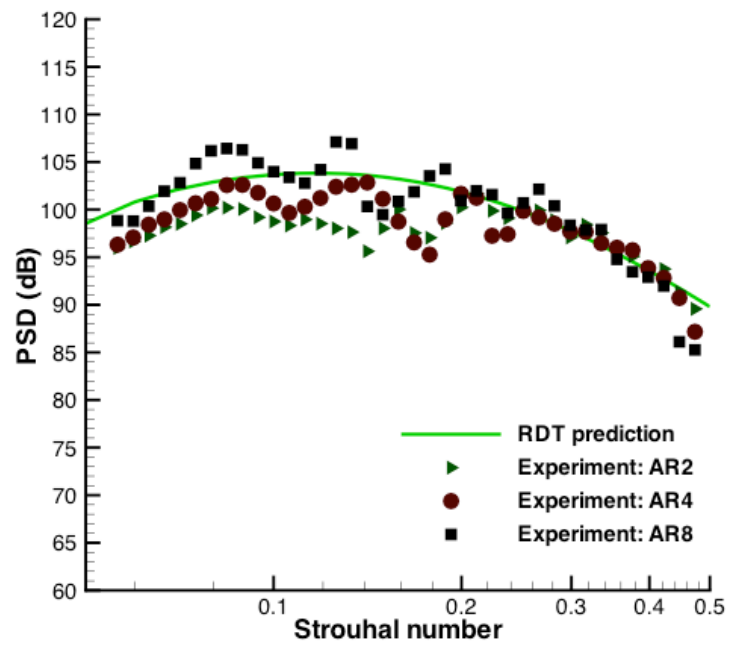

(b). $\theta=75^{\circ}$

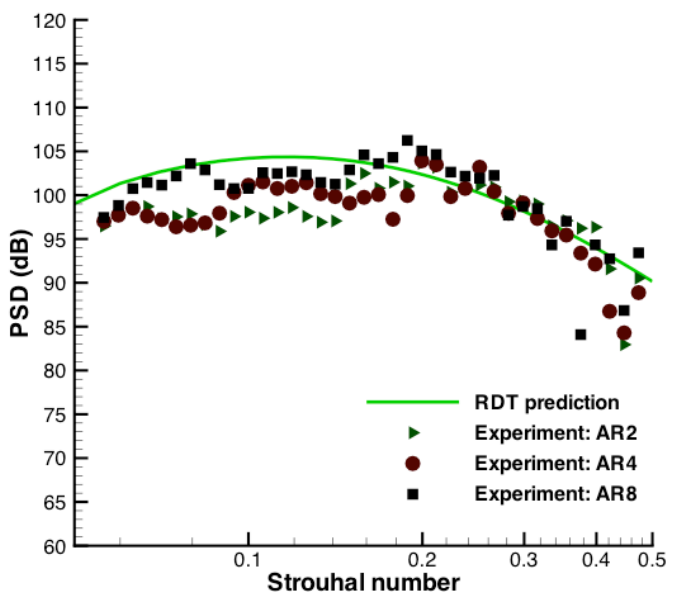

(d). $\theta=45^{\circ}$

Fig. 7- RDT predictions for acoustic Mach number, $M a=0.9$, compared to Power Spectral Density (dB) for various nozzle aspect ratio (AR) rectangular jets [3] at different polar observation angles where $\psi=-90^{\circ}$. Plate location $y_{d} / D_{J}=1.1, x_{d} / D_{J}=5.7$. Source model constants and mean flow for RDT prediction is same as Fig. 5. 


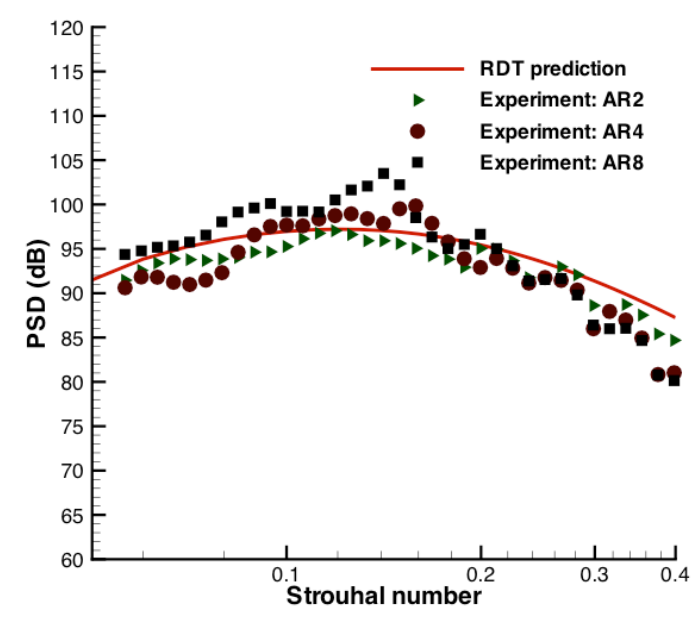

(a). $\theta=90^{\circ}$

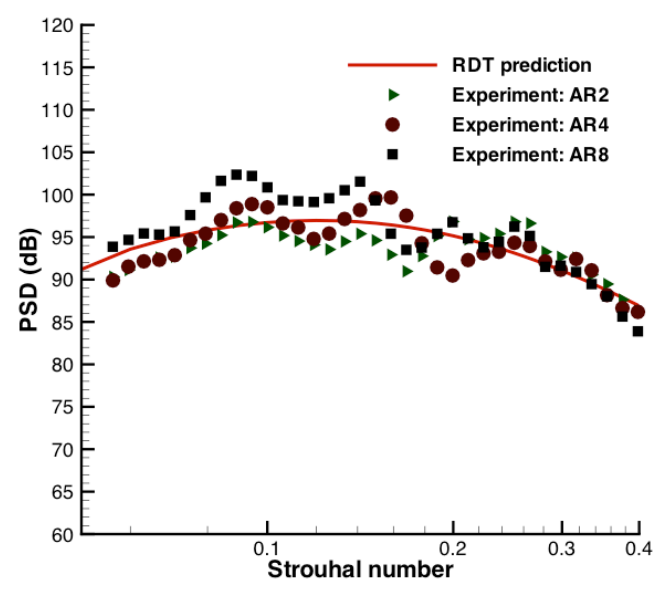

(c). $\theta=60^{\circ}$

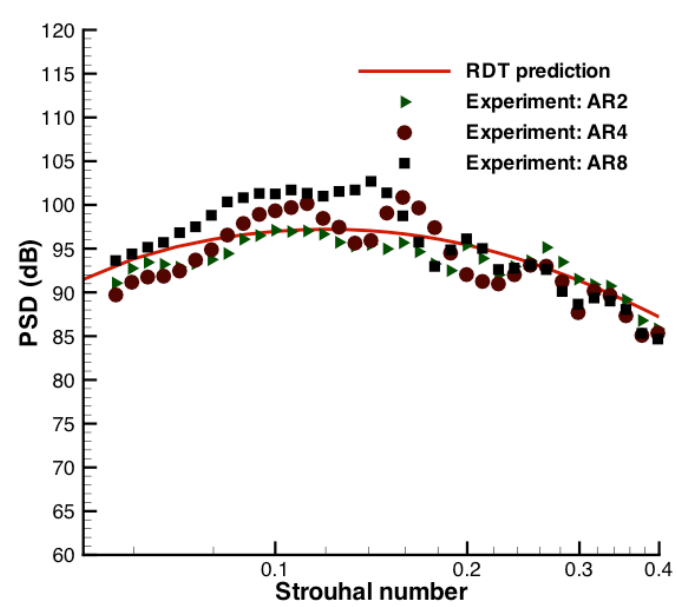

(b). $\theta=75^{\circ}$

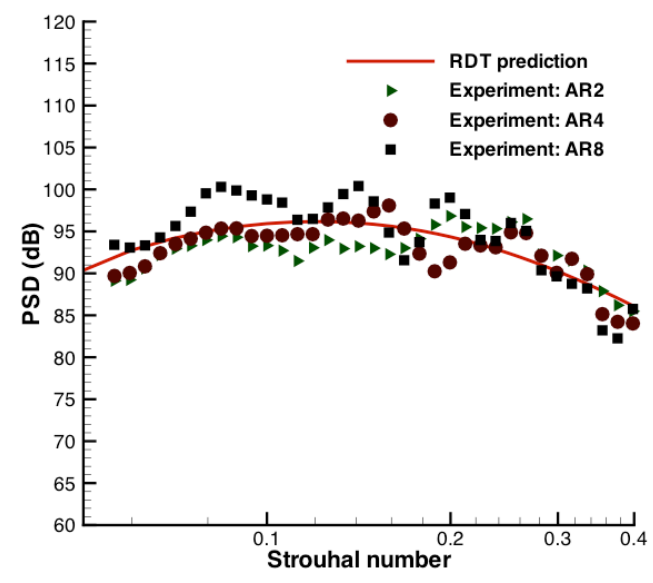

(d). $\theta=45^{\circ}$

Fig. 8- RDT predictions for $M a=0.7$.Same caption as Fig. 7. 


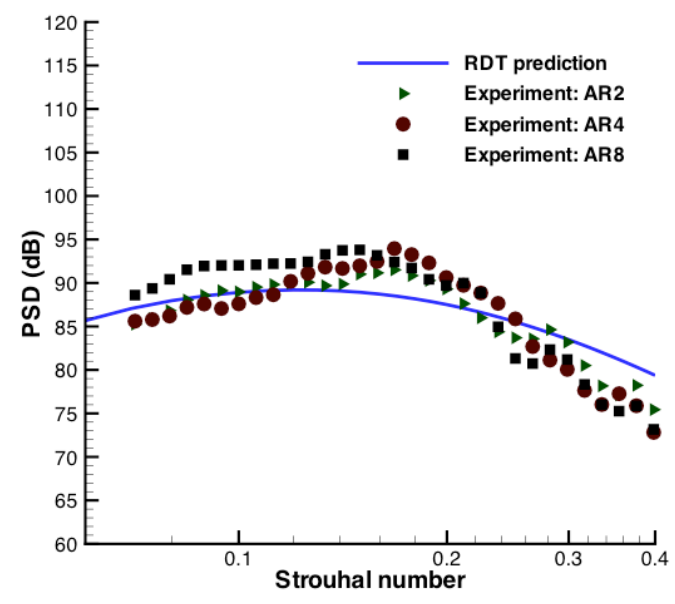

(a). $\theta=90^{\circ}$

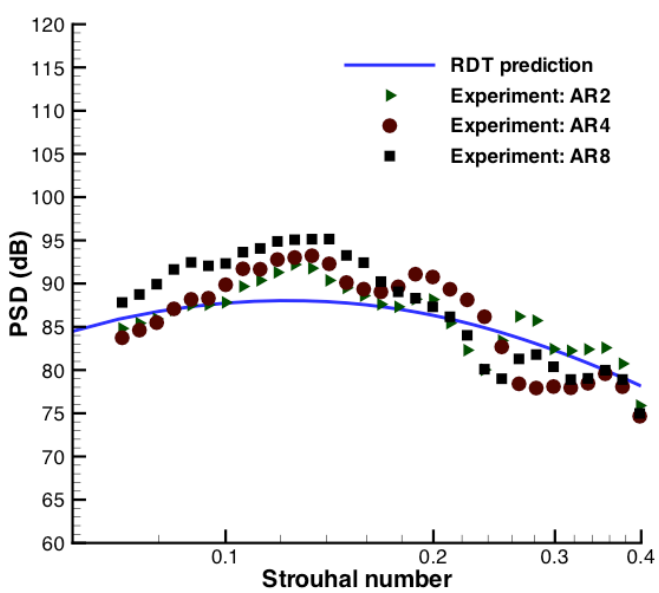

(c). $\theta=60^{\circ}$

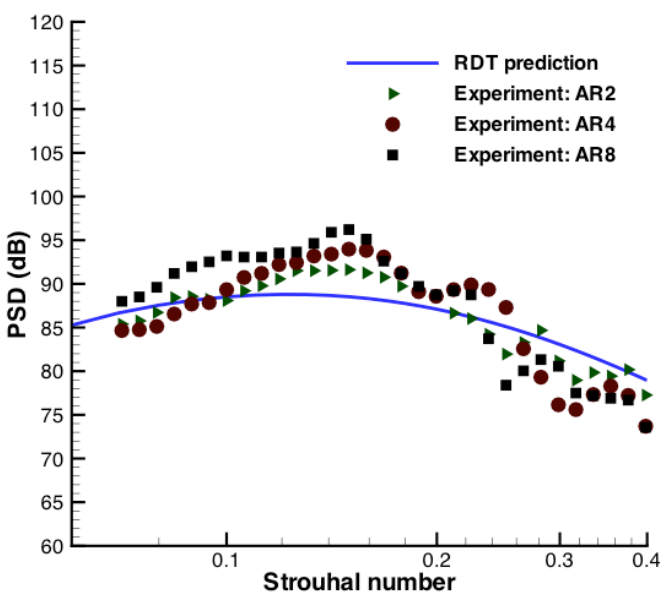

(b). $\theta=75^{\circ}$

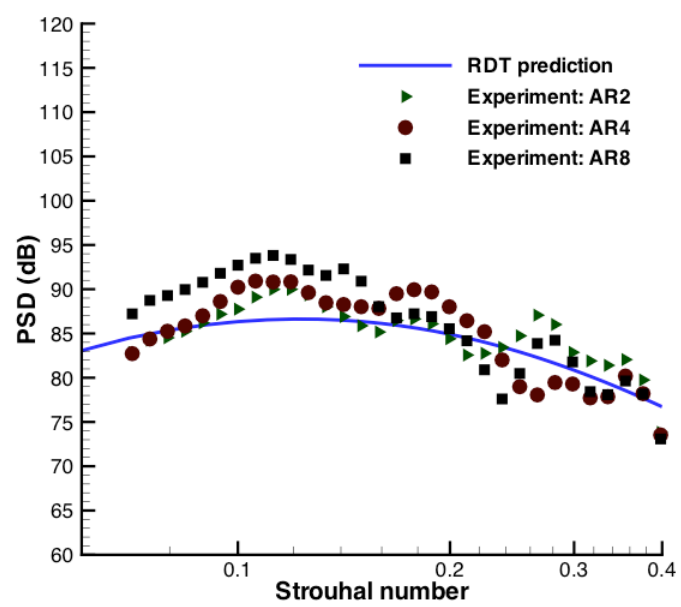

(d). $\theta=45^{\circ}$

Fig. 9- RDT predictions for $M a=0.5$. Same caption as Fig. 7.

\section{REYNOLDS-AVERAGED NAVIER-STOKES (RANS) CALCULATIONS}

It is expected that the turbulence length scales and peak turbulent kinetic energy levels near the trailing edge will change with nozzle aspect ratio and flow conditions ([53]-[56]) and therefore that better agreement with the data could be obtained if these quantities were allowed to vary with the nozzle geometry and jet exit conditions. These variations can be accommodated within the RDT model by using a RANS-based mean flow, turbulent kinetic energy $(k)$ and rate of energy dissipation $(\varepsilon)$ to define the mean velocity profile, length scales and amplitude of the source function, $S\left(y_{2}, \tilde{y}_{2} ; k_{3}^{(s)}, \omega, y_{d}\right)$, near the trailing edge. 
In this section we show results from three-dimensional SolidWorks ${ }^{\circledR}$ Flow Simulations [36]\&[37] of jet flows with acoustic Mach numbers, $M a=(0.5,0.7,0.9)$ through rectangular jet nozzles of aspect ratios, $A R=(2,4,8)$ in the vincity of a flat plate trailing edge. The SolidWorks ${ }^{\circledR}$ Flow Simulation automatic gridding program provides a way to mesh and solve the flow field around complex geometries and is, therefore, rather convenient for the jet surface interaction problem and was used by Bridges \& Wernet [70], amoung others. The inbuilt solver uses adaptive grid refinement on a Cartesian grid and the immersed boundary (IB) method to deal with complex boundaries. Futher detailes of the software can be found in references [36]\&[37]. In the present calculations, the total number of grid points is approximately $2.7 \times 10^{6}$ and run times of the order of $7-8$ hours on a desk-top workstation were found ot be sufficient to obtain the converged mean flow field. The surfaces have adiabatic wall conditions, and the turbulence model (pp. 4-8 of [37]) used is a modified $k-\varepsilon$ with Lam \& Bremhorst [71] damping functions accounting for wall effects. Further information on IB methods and the numerical schemes used to implement them can be found in review paper by Mittal \& laccarino [72].

In Fig. 10 we show the grid convergence properties of the RANS mean axial velocity $U$ and turbulent kinetic energy $k$ for $M a=0.5$ and $A R 8$ for trailing edge location of $\left(x_{d}, y_{d}\right) / D_{J}=(5.7,1.1)$. In Fig. 11, the RANS $U$ and $k$ are compared with hotwire data from Zaman et al. [19] for the same aspect ratio (AR8) and streamwise surface length of 12 inches (the spanwise length was over 48 inches, or $4 \mathrm{feet}$, and extended outside of the solution domain). However, owing to facility constraints in which the measurements were made, the flow conditions are different with very slightly different surface offsets and a lower Mach number. The RANS results have a surface offset of $y_{2} / D_{J}=1.05$, whereas the offset in the experiment is $y_{2} / D_{J}=1.0$. Figure 11 parts $(\mathrm{a})$ and $(\mathrm{b})$ compare profiles of the mean streamwise velocity and turbulent kinetic energy, respectively, normalized by the ideally-expanded jet exit velocity, $U_{J}$, from the RANS and hot-wire data. Figure 11 (c) and (d) shows this comparison along the jet centerline. There was no data available for the centerline TKE distribution. 


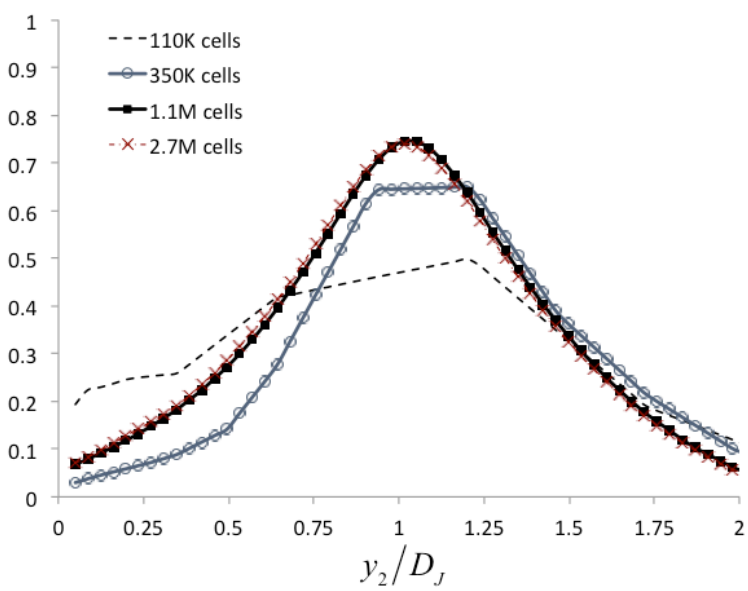

(a). Mean velocity, $U / U_{J}$

\begin{tabular}{|c|c|c|c|}
$\begin{array}{c}\text { Grid resolution } \\
\text { study }\end{array}$ & Number of Cells & Iterations & $\begin{array}{c}\text { Elapsed time } \\
\text { (hours) }\end{array}$ \\
\hline Initial Grid & $0.11 \mathrm{M}$ & 600 & $0: 40$ \\
\hline 1st Refinement & $0.35 \mathrm{M}$ & 800 & $1: 19$ \\
\hline 2nd Refinement & $0.57 \mathrm{M}$ & 1000 & $2: 13$ \\
\hline 3rd Refinement & $1.1 \mathrm{M}$ & 1250 & $3: 55$ \\
\hline 4th Refinement & $2.8 \mathrm{M}$ & 1477 & $7: 33$ \\
\hline
\end{tabular}

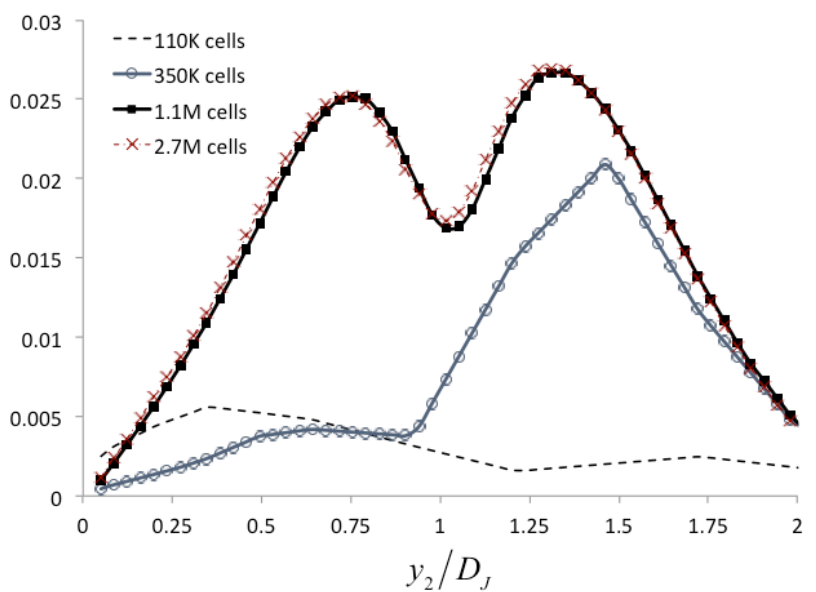

(b). Turbulent Kinetic Energy, $\mathrm{k} / U_{J}^{2}$

Fig. 10 - Grid convergence for $U / U_{J}$ and $k / U_{J}^{2}$ obtained by RANS SolidWorks ${ }^{\circledR}$ calculation at the trailing edge location, $y_{d} / D_{J}=1.1, x_{d} / D_{J}=5.7$, $D_{J}=2.12 "$ of $M a=0.5$ and $A R 8$ nozzle.

The results in Fig. 11 show that, while the shapes of the axial velocity and turbulent kinetic energy distributions are qualitatively consistent, the peak turbulent kinetic energy is overpredicted by about 35\% compared to PIV data at the trailing edge location. These differences are consistent with other RANS CFD codes, such as the Wind-US code [73]. Cross-stream distributions of mean axial velocity and turbulent kinetic energy shown in Fig. 12 near the surface trailing edge $\left(x_{d} / D_{J}=5.7\right.$ in Fig. 3), compare favorably with the experimental results. Note that the arrows in Fig. 12 are in the direction of decreasing magnitude. Notwithstanding the limited amount of experimental data we have at our disposal with which to perform an adequate comparison, the agreement is good enough for use in our prediction model. 


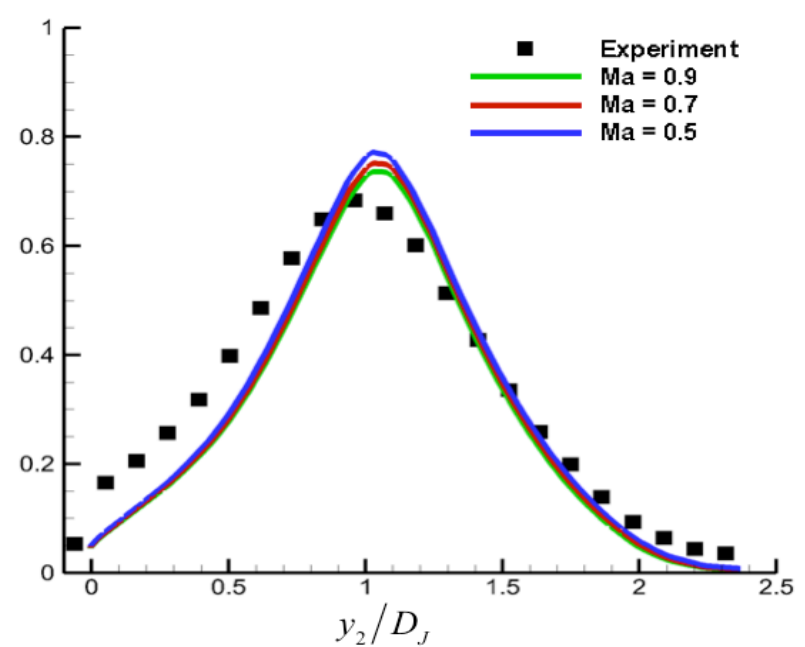

(a). Mean velocity, $U / U_{J}$

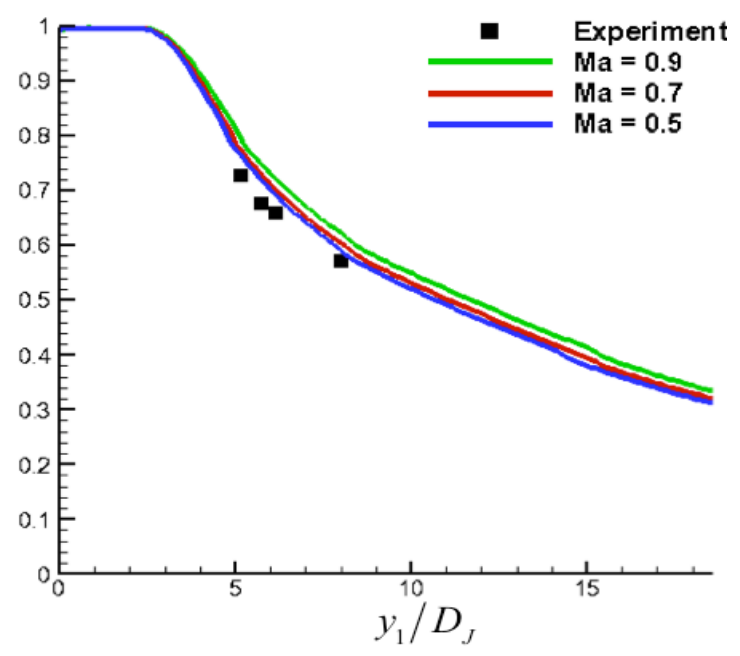

(c). Centreline $U / U_{J}$

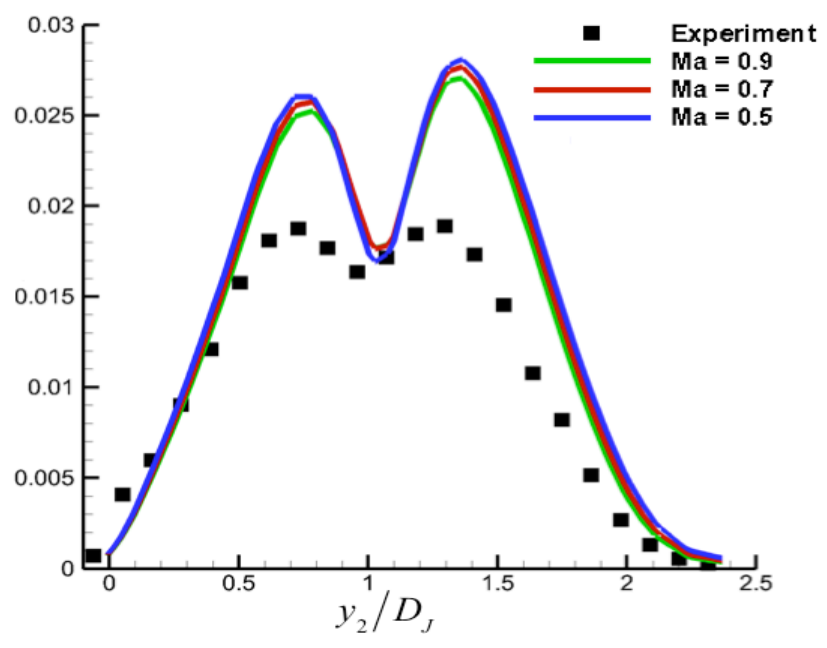

(b). Turbulent Kinetic Energy, $k / U_{J}^{2}$

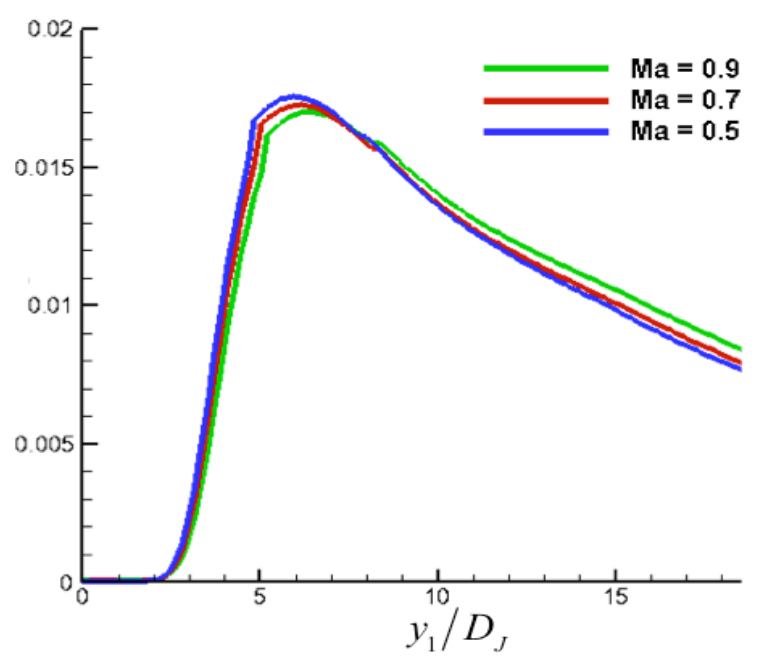

(d). Centerline $k / U_{J}^{2}$

Fig. 11- Comparison of normalized mean flow and turbulent kinetic energy obtained by RANS SolidWorks ${ }^{\circledR}$ calculation at the trailing edge location, $y_{d} / D_{J}=1.1, x_{d} / D_{J}=5.7, D_{J}=2.12 "$, with low Mach number \& high aspect ratio experiment reported in Zaman et al. [19], where jet Mach number is $\mathbf{M j}=\mathbf{0 . 2 2}$ and $A R=8$. 


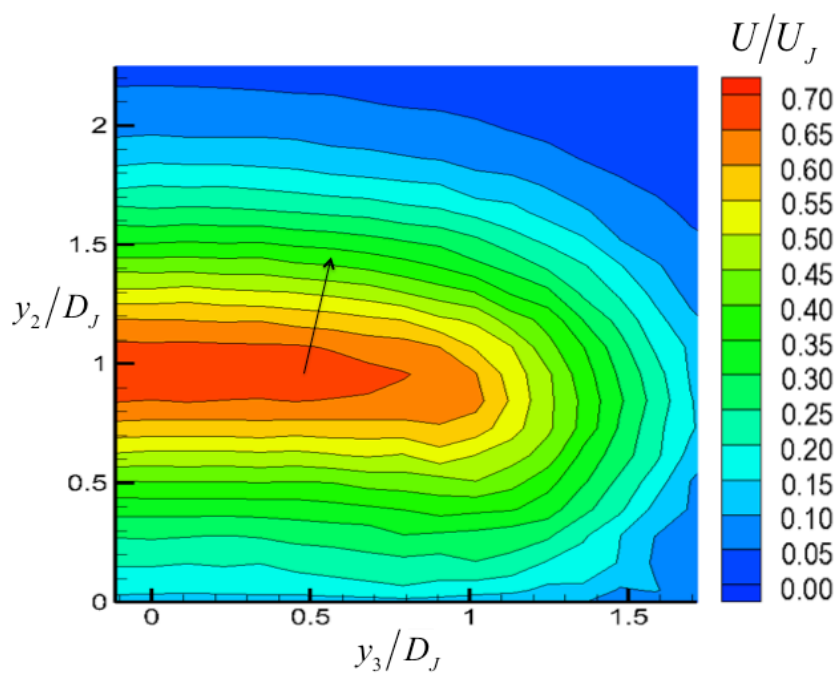

(a). $U / U_{J}$ : Zaman et al. [19].

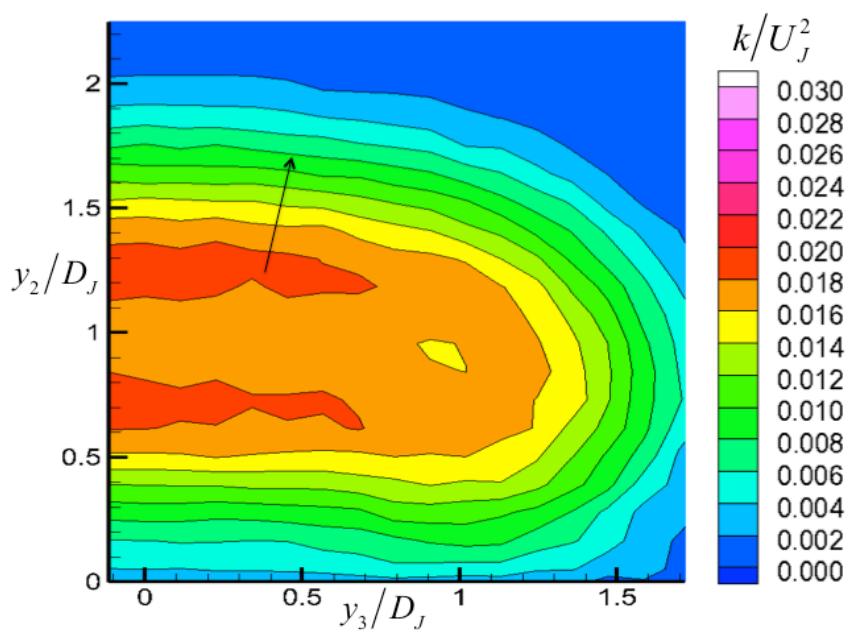

(c). $k / U_{J}^{2}:$ Zaman et al. [19].

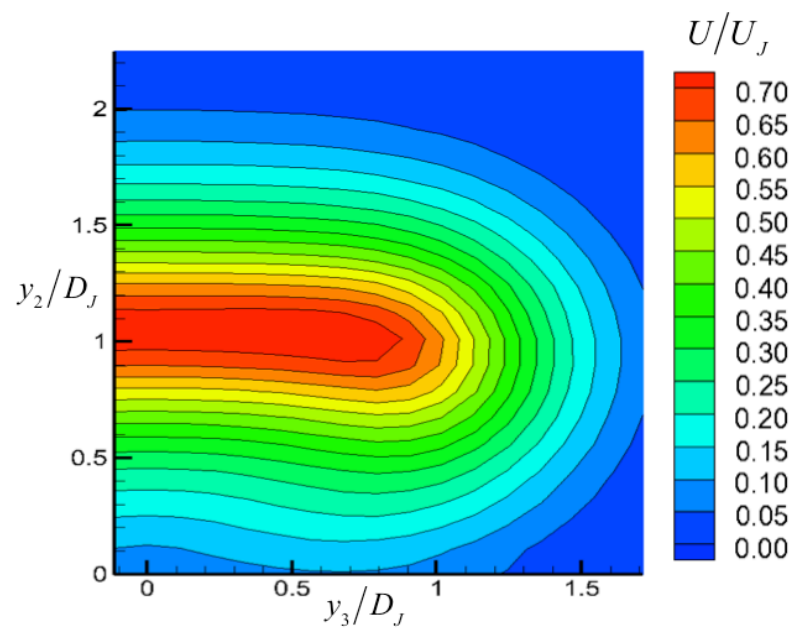

(b). $U / U_{J}:$ RANS calculation.

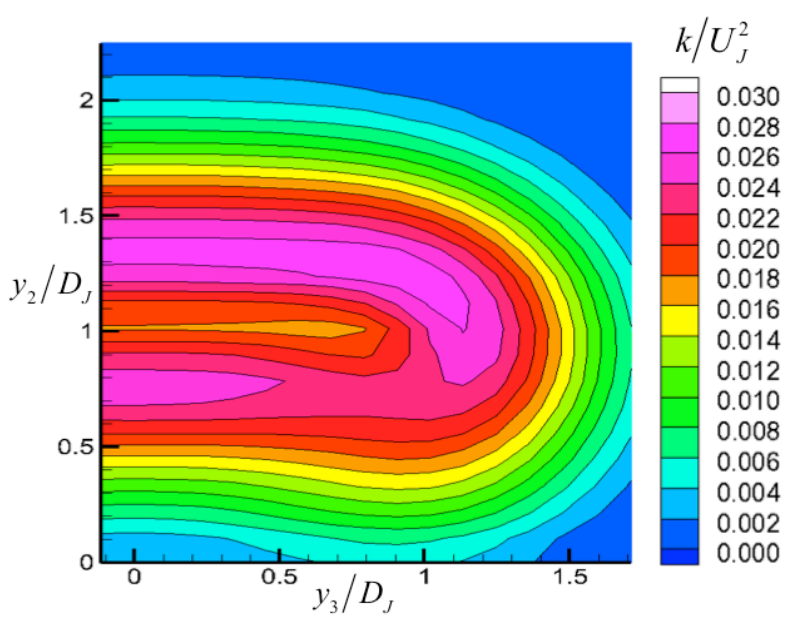

(d). $k / U_{J}^{2}:$ RANS calculaton.

Fig. 12- Comparison of Normalized mean velocity and turbulent kinetic energy obtained by RANS SolidWorks ${ }^{\circledR}$ calculation and experiments reported in Zaman et al. [19] for same trailing edge location as the caption in Fig. 8. Arrows in direction of decreasing magnitude.

Fig. 13 shows values of the normalized RANS-based turbulent kinetic energy, $k$, and length scale, $L_{R A N S}=k^{3 / 2} / \varepsilon$, at the axial location corresponding to the trailing edge of the plate and a transverse location at the peak of the mean axial velocity profile location at the center of the span for $M a=(0.5,0.7,0.9)$ and $(A R 2, A R 4, A R 8)$. The results show that these normalized RANS-based quantities decrease as the nozzle $A R$ reduces for any given acoustic Mach number. On the other hand, the variation of these normalized scales is relatively insignificant at a fixed $A R$ and varying $M a$. The SolidWorks mean velocity field appeared to 
be contaminated with local flow separation regions for the $A R 2$ nozzle so that meaningful noise predictions were not possible. In section 6 , therefore, we show only noise predictions for the $A R 8$ and $A R 4$ nozzles.

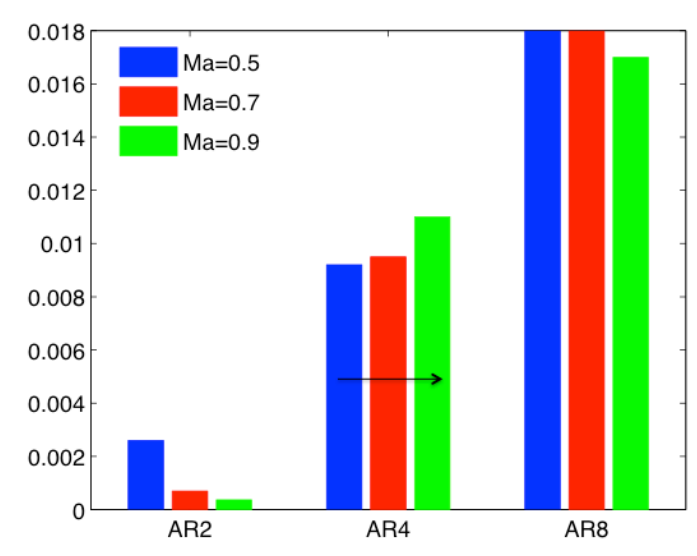

(a). $k / U_{J}^{2}$

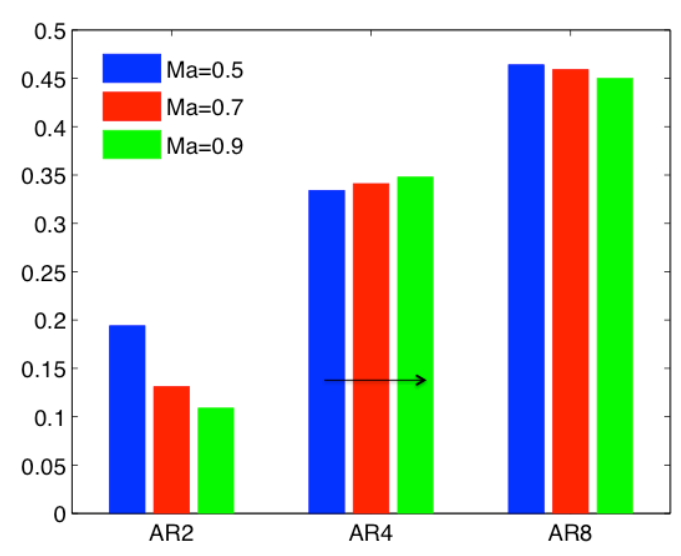

(b). $L_{\text {RANS }} / D_{J}$

Fig. 13- Variation of normalized turbulent kinetic energy and length scale obtained by RANS calculation with Nozzle Aspect Ratio (AR) and Acoustic Mach number (Ma). Arrows in direction of increasing Ma.

\section{RANS-BASED JET-SURFACE INTERACTION NOISE PREDICTIONS}

We use the RANS solutions described in the last section within the RDT model in a similar way to acoustic analogy approaches [38]; i.e. by defining the lengthscales and amplitude function in Eq. (17) as follows, $l_{n}=c_{n}\left(k^{3 / 2} / \varepsilon\right), n=0,1 \& 3$ and $\Psi_{0}=c_{\Psi} \bar{\rho} k$, where $\left(c_{n}, c_{\Psi}\right)$ are empirical parameters. The streamwise mean flow profile, $U\left(y_{2}\right) / U_{J}$, is now obtained directly from the RANS calculation at the axial location corresponding to the trailing edge of the plate. When choosing the scaling coefficents, particular consideration was given to the acoustic Mach number $M a=0.9$ case (since the jet-surface interaction has a clear spectral peak at higher speeds [1]-[3]), for $A R 8$ (since the theory is directly applicable here) and at the polar angle $\theta=90^{\circ}$ where the interaction noise is greatest in the forward arc.

In the following set of predictions the values for the length scale parameters $\left(c_{0}, c_{1}, c_{3}\right)$, the amplitude, $c_{\Psi}$, the convection velocity $U_{c}$ and the normalized parameter, $b_{0} / y_{d}$ are kept fixed in all (24 prediction locations for $y_{d} / D_{J}=1.1$ ) cases at a fixed trailing edge location, $y_{d} / D_{J}$. However, owing to expected changes in turbulence properties with aspect ratio, we decreased the geometric length $L_{3} / D_{J}$ to account for the smaller spanwise extent of the turbulence in the smaller aspect ratio case. In particular, we took $L_{3} / D_{J}=20$ for $A R 8$ and $L_{3} / D_{J}=10 A R 4$. Moreover, the results of many numerical experiments indicated 
that closer agreement to the data could be achieved when the de-correlation region reduced with aspect ratio.

The coefficients for the source model in Eq. (15) are given in Table 1 and in Fig. 14 we show the normalized auto-correlation function for Eq. (14), using models Eqs. (15) \& (16), corresponding to the parameters $\left(a_{0}, a_{1}, a_{2}\right)$ chosen for $A R 4$ and $A R 8$. The effect is to decrease the size of the decorrelation region at smaller aspect ratio. The remaining coefficients in Eq. (18) we set at $\left(c_{0}, c_{1}, c_{3}\right)=(1.4,0.021,0.022), U_{c}=0.65 U_{d}, c_{\Psi}=1.5$ and $b_{0} / y_{d}=0.65$.

\begin{tabular}{||c||c|c|l|}
\hline \hline \begin{tabular}{c}
\hline TEST \\
CASES
\end{tabular} & $y_{d}=1.1$ & $y_{d}=1.7$ & $\begin{array}{l}\text { Parameters } \\
\text { in Eq. (26) }\end{array}$ \\
\hline \hline & $M a=0.9$ & $M a=0.9$ & $\left(a_{0}, a_{1}, a_{2}\right)=$ \\
AR8 & $M a=0.7$ & $M a=0.7$ & $(0.52,0.96,-0.15)$ \\
& $M a=0.5$ & & \\
\hline \hline & $M a=0.9$ & $M a=0.9$ & $\left(a_{0}, a_{1}, a_{2}\right)=$ \\
AR4 & $M a=0.7$ & $M a=0.7$ & $(0.78,0.92,-0.05)$ \\
& $M a=0.5$ & & \\
\hline \hline
\end{tabular}

Table 1 - Summary of RANS test cases along with de-correlation parameters in Eq. (15) for each aspect ratio.

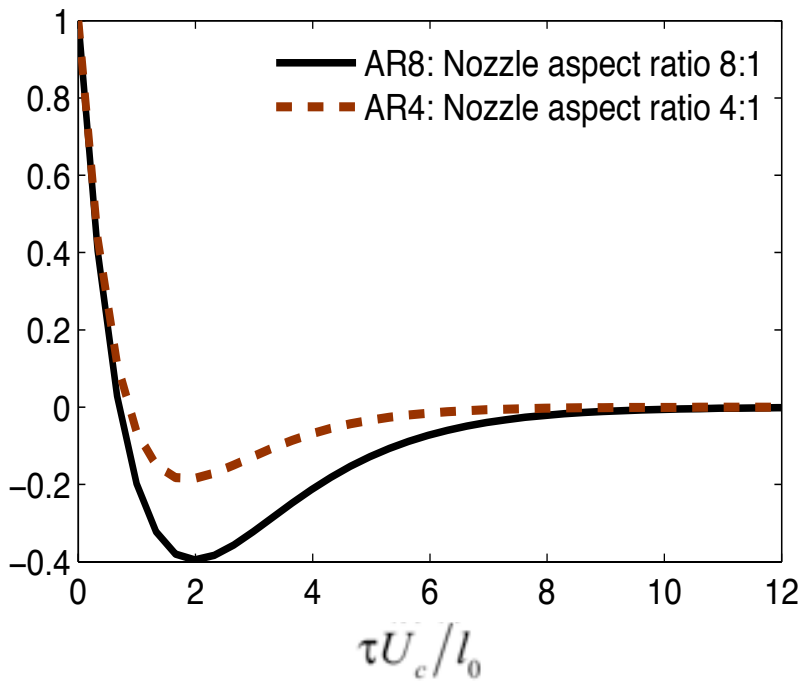

(a). $\eta_{1} / l_{1}=0$,

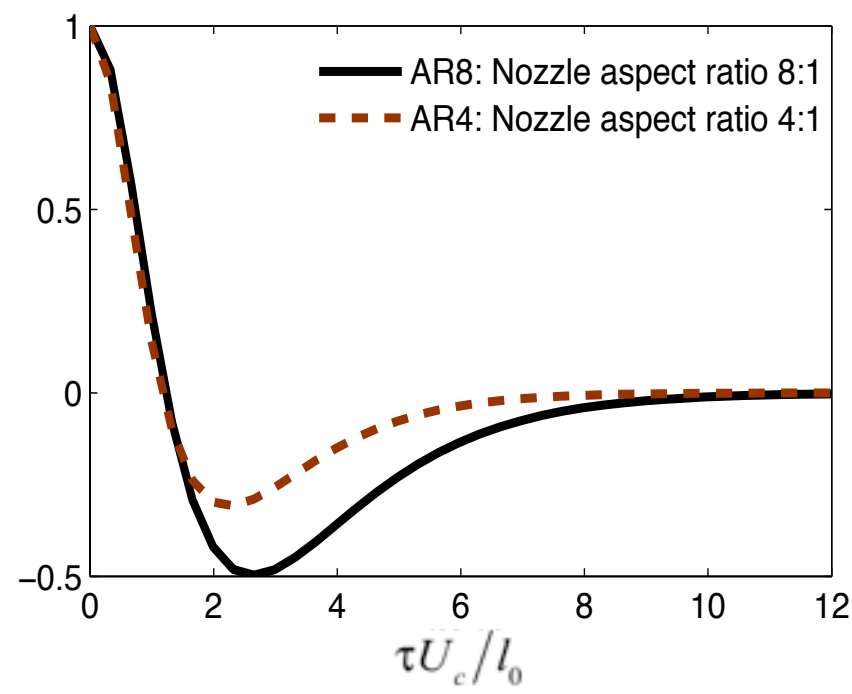

(b). $\eta_{1} / l_{1}=1.0$.

Fig. 14 Auto-correlation of Eqs. (15) with $\left(\eta_{3}=0\right)$ for AR4 \& AR8.

In Figs. 15-17 we show the RANS-based RDT predictions for $M a=(0.5,0.7,0.9)$, respectively for nozzle aspect ratio $A R 8$ across a polar angle range of $\theta=\left(45^{\circ}, 60^{\circ}, 75^{\circ}, 90^{\circ}\right)$ at the 
azimuthal location below the plate $\left(\psi=-90^{\circ}\right)$. As we found earlier, with the analytically based mean flow model, appropriate choices of the parameters $\left(a_{1}, a_{2}\right)$ in Eq. (15) provide a low-frequency roll-off that is closer to the data reported in [3]. Comparing Figs. 7-9 with 1517 , shows improvement in the acoustic predictions using the RANS-based model, especially for $M a=(0.7,0.9)$. It should be remembered, however, that since the model is based on a leading order asymptotic approach, it is expected to predict the dominant spectral features of the JSI noise only. We have not explicitly included the intereference effects associated with jet /JSI noise coupling (which we believe are responsible for the oscillations in the data, see Sec. 7). Note that the level of agreement in most cases lies within those oscillations; they are better at larger polar angles in the forward arc and higher Mach numbers.

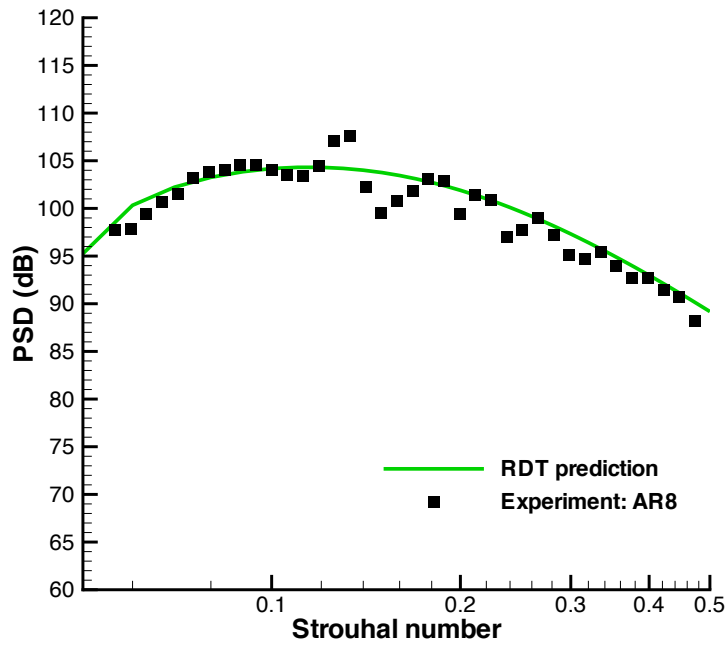

(a). $\theta=90^{\circ}$

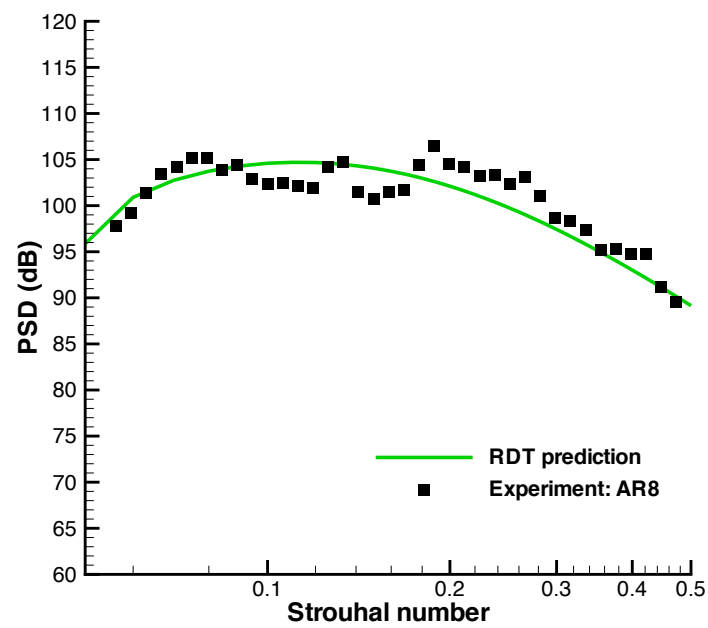

(c). $\theta=60^{\circ}$

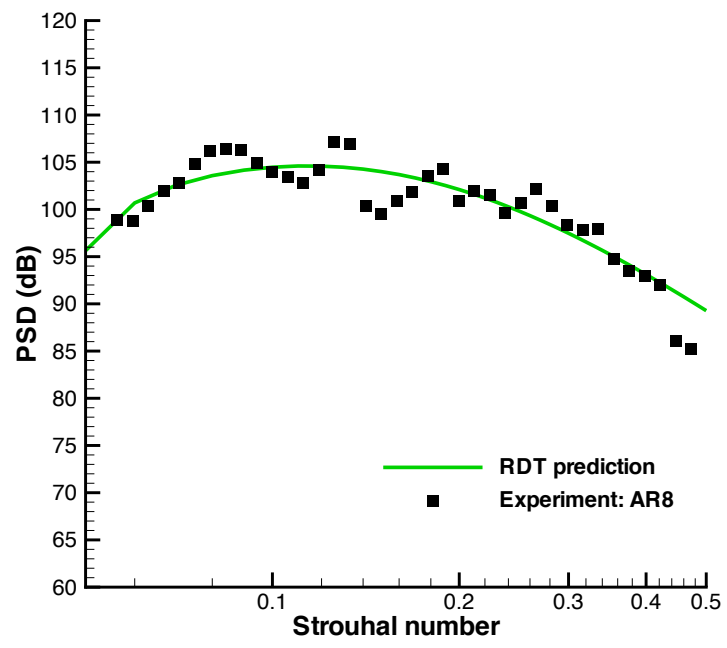

(b). $\theta=75^{\circ}$

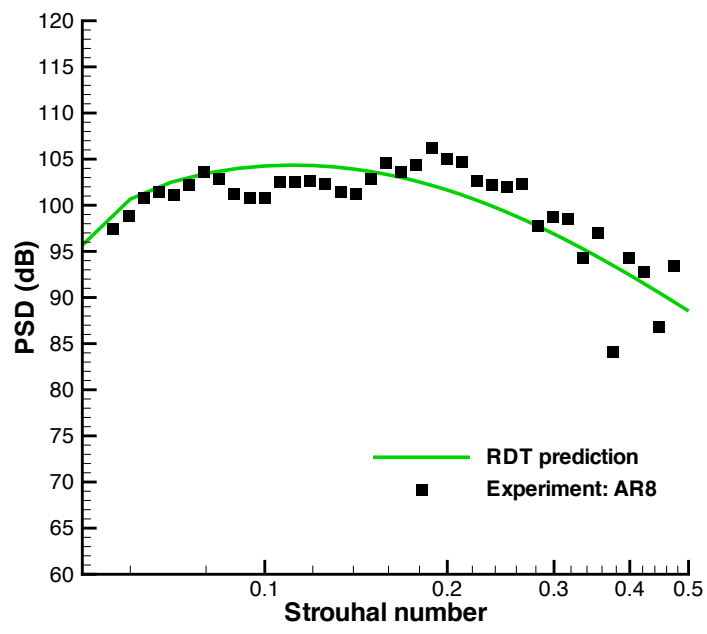

(d). $\theta=45^{\circ}$

Fig. 15 RANS-based RDT prediction for acoustic Mach number $M a=0.9$ and $A R=8$ compared to noise data [3] at different polar observation angles $\left(\psi=-90^{\circ}\right)$. Plate location $y_{d} / D_{J}=1.1, x_{d} / D_{J}=5.7$. 


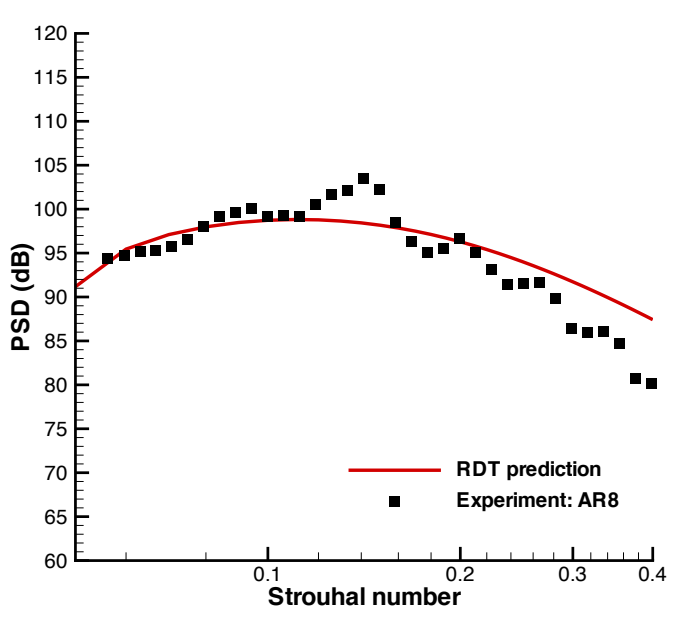

(a). $\theta=90^{\circ}$

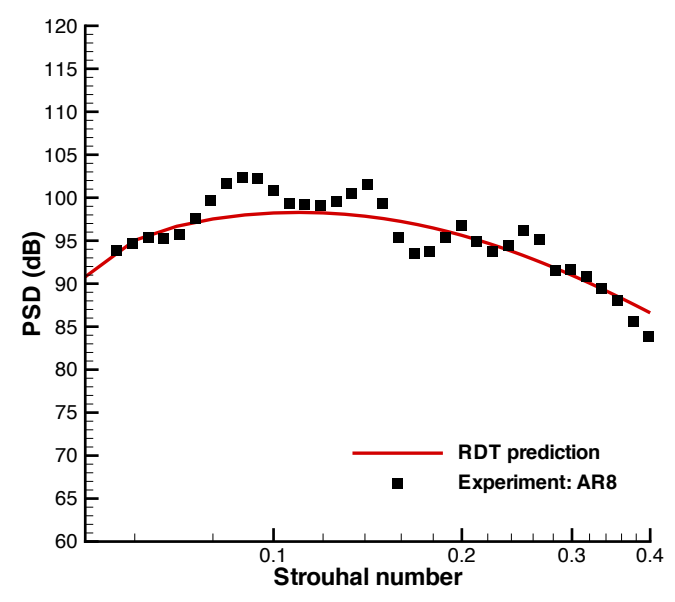

(c). $\theta=60^{\circ}$

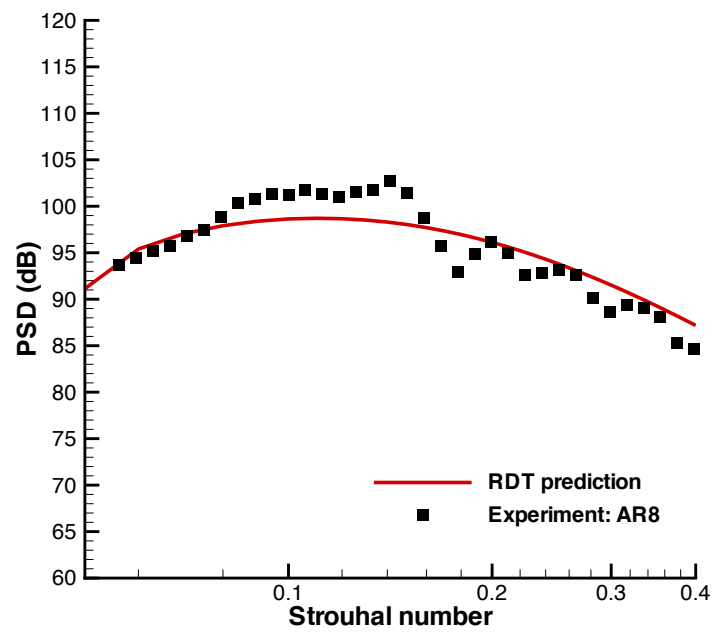

(b). $\theta=75^{\circ}$

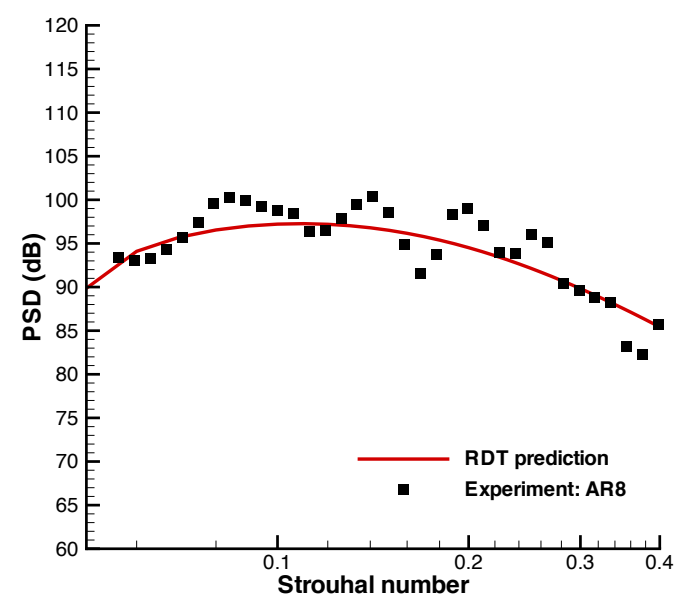

(d). $\theta=45^{\circ}$

Fig. 16 RANS-based RDT prediction for $M a=0.7$ and $A R=8$. Plate location $y_{d} / D_{J}=1.1, x_{d} / D_{J}=5.7$. 


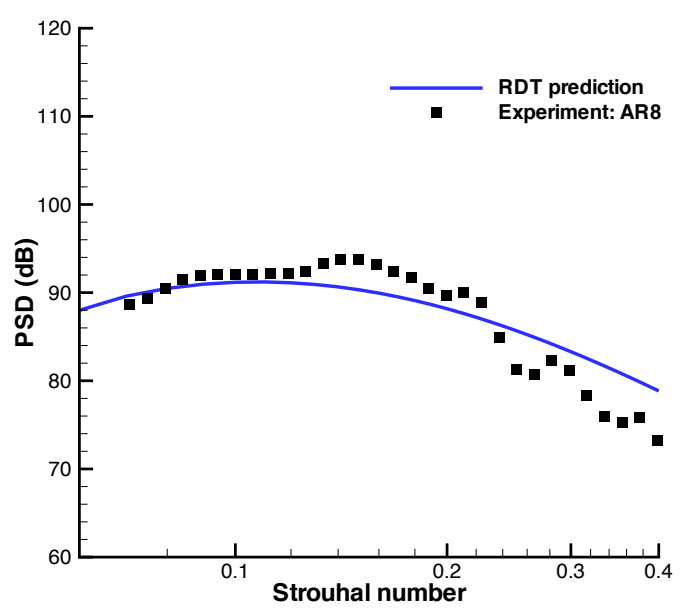

(a). $\theta=90^{\circ}$

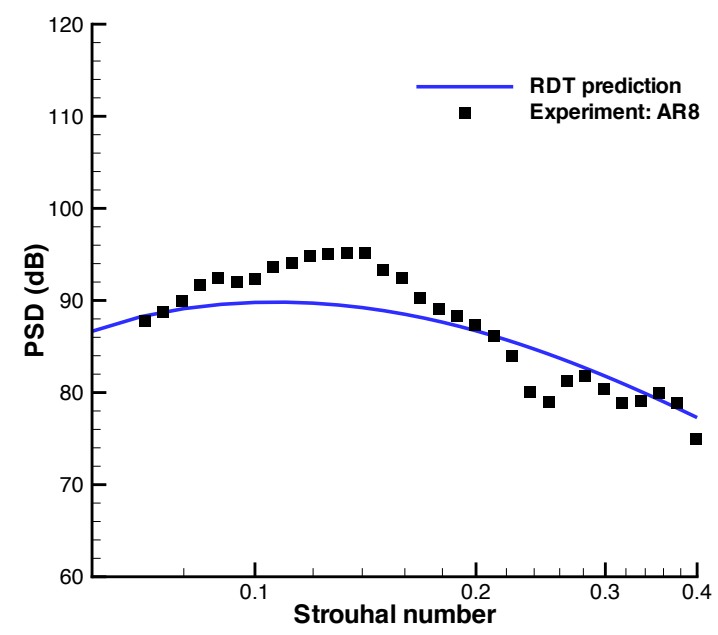

(c). $\theta=60^{\circ}$

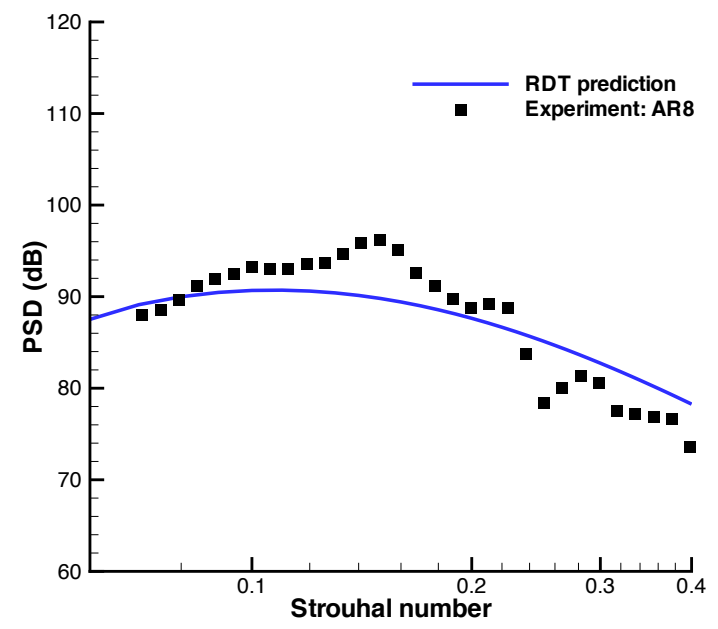

(b). $\theta=75^{\circ}$

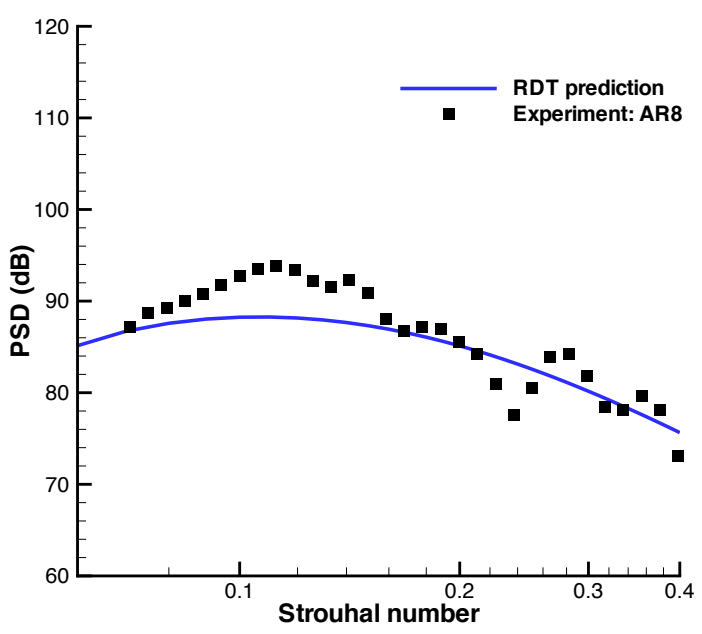

(d). $\theta=45^{\circ}$

Fig. 17 RANS-based RDT prediction for $M a=0.5$ and $A R=8$. Plate location $y_{d} / D_{J}=1.1, x_{d} / D_{J}=5.7$.

\subsection{Noise predictions for AR4 nozzle}

To assess the robustness of our model, in Fig. 18 we show the RANS-based RDT predictions for $M a=(0.5,0.7,0.9)$ for nozzle aspect ratio $A R 4$ at the same plate location as Figs. 15-17, $y_{d} / D_{J}=1.1, x_{d} / D_{J}=5.7$. Since the results in Figs. 13-15 show that there is little polar directivity, we show angles, $\theta=\left(45^{\circ}, 90^{\circ}\right)$, only. Predictions using the planar mean flow approximation for $A R 4$ are quite good for $M a=0.9$, but there is a systematic underprediction of the measured edge noise with decreasing Mach number. We note that the frequency band over which the noise radiates is much smaller for lower Mach numbers. This underprediction is, perhaps, not unexpected since, firstly the theory applies strictly to a planar mean flow and its application to lower aspect ratios must be treated as a first approximation and, moreover, the parameters in Eq. (18) were set for $M a=0.9$ in mind. 

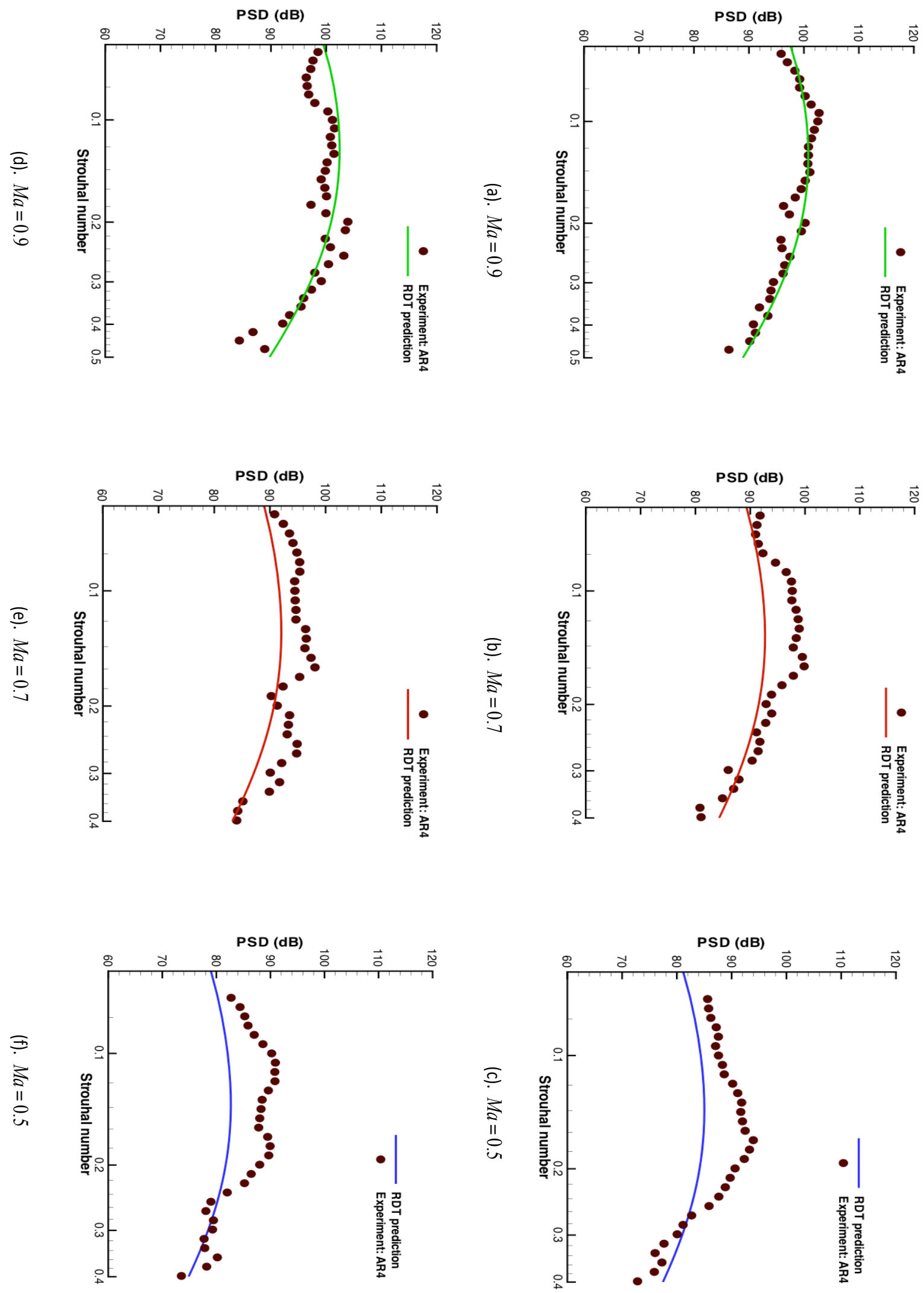

Fig. 18 RANS-based RDT prediction for $A R=4\left(\mathrm{a}-\mathrm{c}: \theta=90^{\circ}\right)$ and (d-f: $\left.\theta=45^{\circ}\right)$. Same edge location as Fig. 15. 
6.2 Variation of trailing edge location relative to jet center-line

Data in Fig. 8 of [3] show that the edge noise is most significant when the plate is placed near the location $x_{d} / D_{J}=5.7$ for $A R 4$ and $A R 8$ nozzles. Interestingly, this lies in the region near the end of jet's potential core (that usually lies anywhere between $x_{d} / D_{J}=4$ and $x_{d} / D_{J}=10$ in the absence of a plate) which is thought to be the location of the source of maximum jet noise [38]. In Fig. 19 we show noise predictions for a trailing edge location slightly further away in the transverse direction $\left(y_{d} / D_{J}=1.7\right)$ compared with the previous calculations. We present these result to further test the robustness of the model. Note that the parameters have all been kept fixed at their values used in the previous RANS-based predictions. Results are shown for polar angles of ninety (solid curves) and forty-five (dashed curves) degrees. Although, at very low frequencies (up to the peak noise) the predictions agree well with data, there is deterioration as the Strouhal number increases.

Fig. 20 shows the ratios of TKE and length scale $L_{R A N S}=k^{3 / 2} / \varepsilon$ obtained from the RANS calculation with varying $x_{d} / D_{J}$ at fixed $y_{d} / D_{J}$, (a) and vice versa, (b), to their respective values at the plate location $x_{d} / D_{J}=5.7$ and $y_{d} / D_{J}=1.1$, where all previous sound predictions have been perfomed. The results show that the maximum TKE level occurs for the streamwise location $x_{d} / D_{J}=5.7$, perhaps explaining the larger edge noise for this geometry. The RDT calculation at location $x_{d} / D_{J}=3.8$ is also shown in this figure. The predictions (with coefficients fixed as in table 1) are in reasonably good agreement with the data, except for the very lowest frequencies where the predicted spectrum decays too rapidly owing, most likely, to the choice of the source parameters $\left(a_{1}, a_{2}\right)$. Overall, the results in Figs. 15-20 are encouraging given that the model parameters in Eq. (18) have been kept fixed over the large number of test cases (40 in total, at both edge locations $y_{d} / D_{J}$ in Table 1 ) for which we make comparisons. 


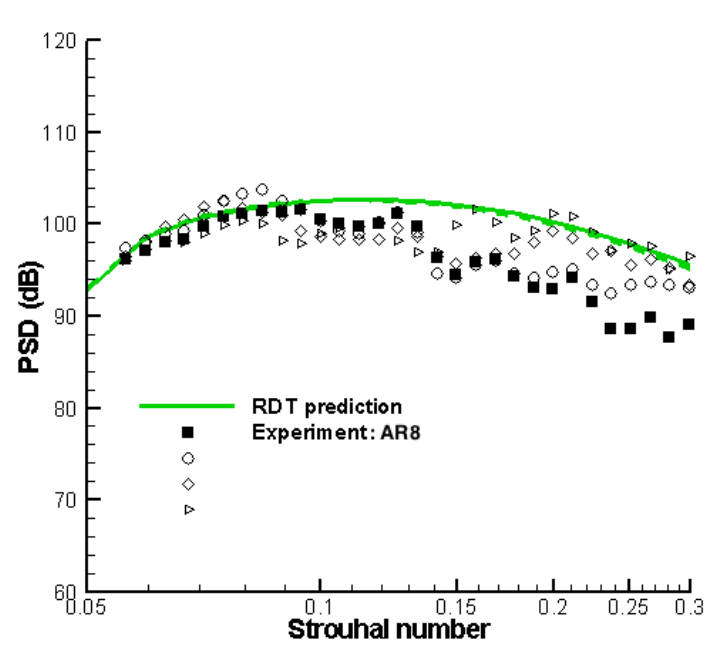

(a). $M a=0.9$

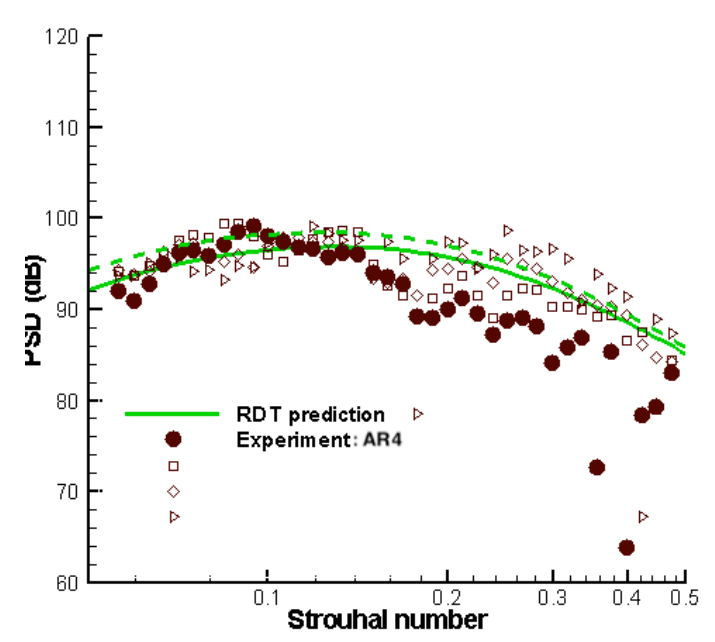

(c). $M a=0.9$

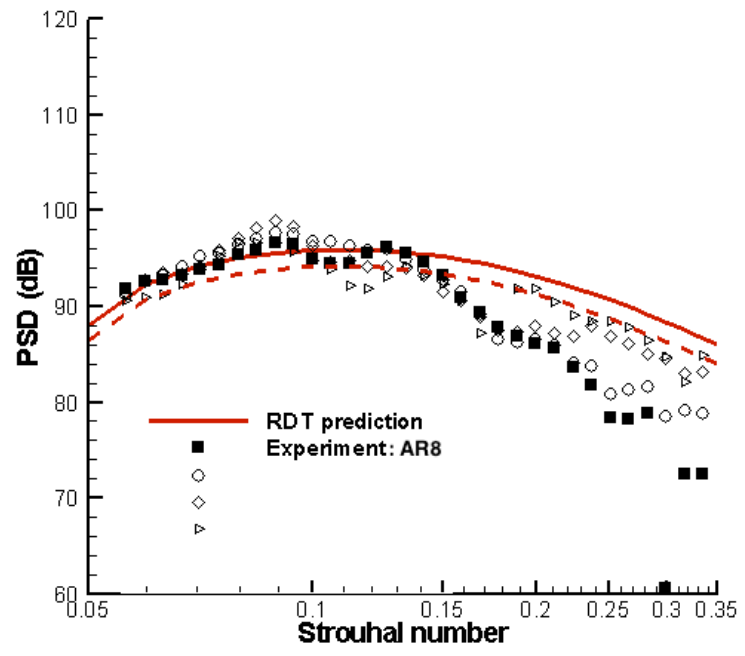

(b). $M a=0.7$

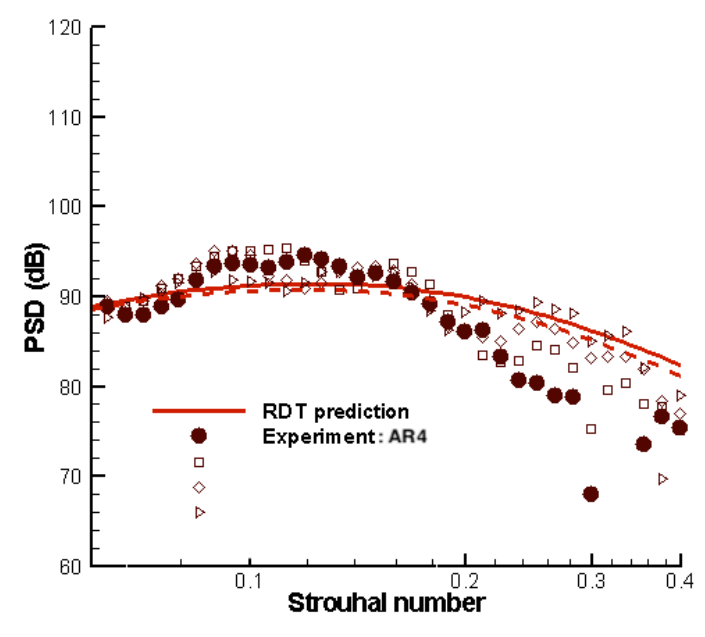

(d). $M a=0.7$

Fig. 19 RANS-based RDT predictions for plate location $y_{d} / D_{J}=1.7, x_{d} / D_{J}=5.7$ compared to data [3] at different polar observation angles. Solid line/dashed line is prediction at $\theta=90^{\circ} / 45^{\circ}$. Solid square is data at $\theta=90^{\circ}$, open circle, diamond, right hand triangle is $\theta=\left(75^{\circ}, 60^{\circ}, 45^{\circ}\right)$ respectively. The azimuthal angle is

$$
\psi=-90^{\circ} \text {. }
$$




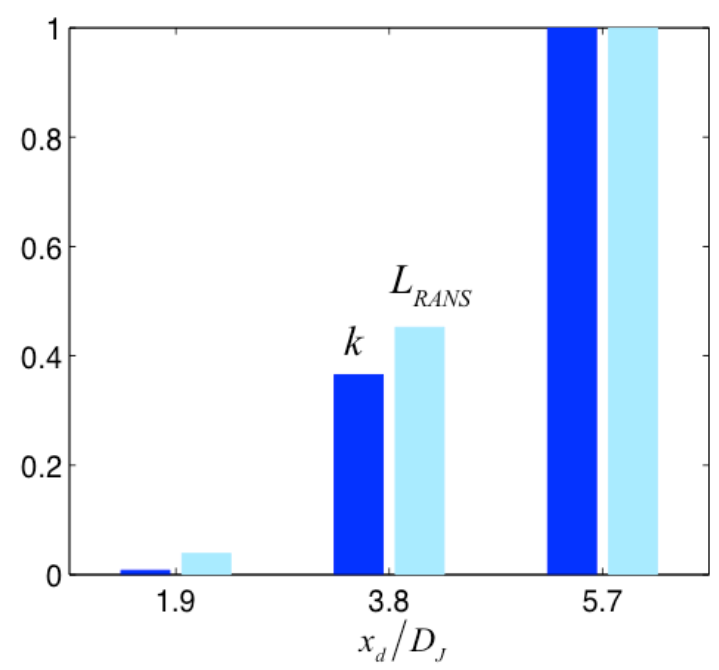

(a). $y_{d} / D_{J}=1.1$

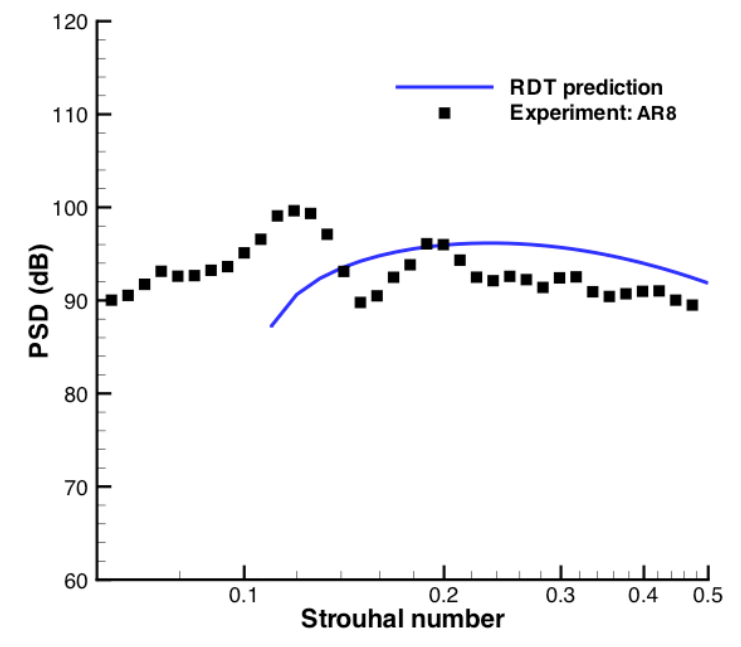

(c). $\theta=90^{\circ}$

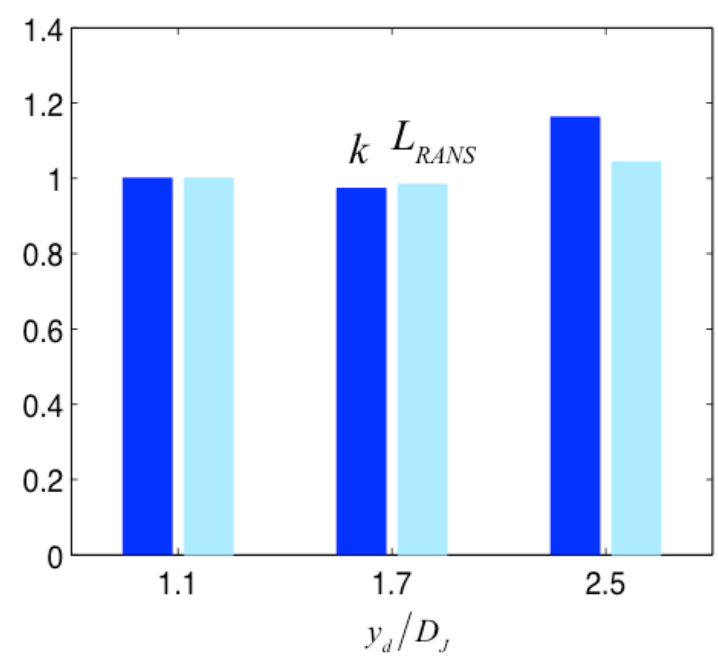

(b). $x_{d} / D_{J}=5.7$

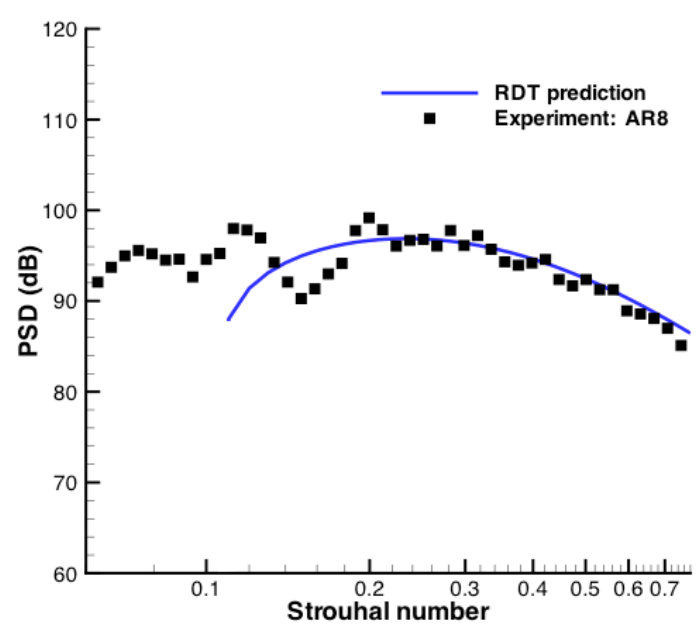

(d). $\theta=60^{\circ}$

Fig. 20 RANS-based turbulence properties (a \& b) for $A R=8, M a=0.9$ and RDT prediction for same jet flow with plate located at $x_{d} / D_{J}=3.8$ and $y_{d} / D_{J}=1.1$. Comparison made against data in [3] at $\psi=-90^{\circ}$.

\subsection{Applicability of RDT-model for closely positioned surfaces}

In Fig. 21 we assess the capability of the RANS-based RDT predictions for closely alligned plates with trailing edge at $y_{d} / D_{J}=(0.25,0.4)$ and $x_{d} / D_{J}=5.7$. For these cases we consider, $M a=0.9$ only and $A R 8$ (where the theory is directly applicable only). The polar angle range is $\theta=\left(75^{\circ}, 90^{\circ}, 105^{\circ}\right)$. 

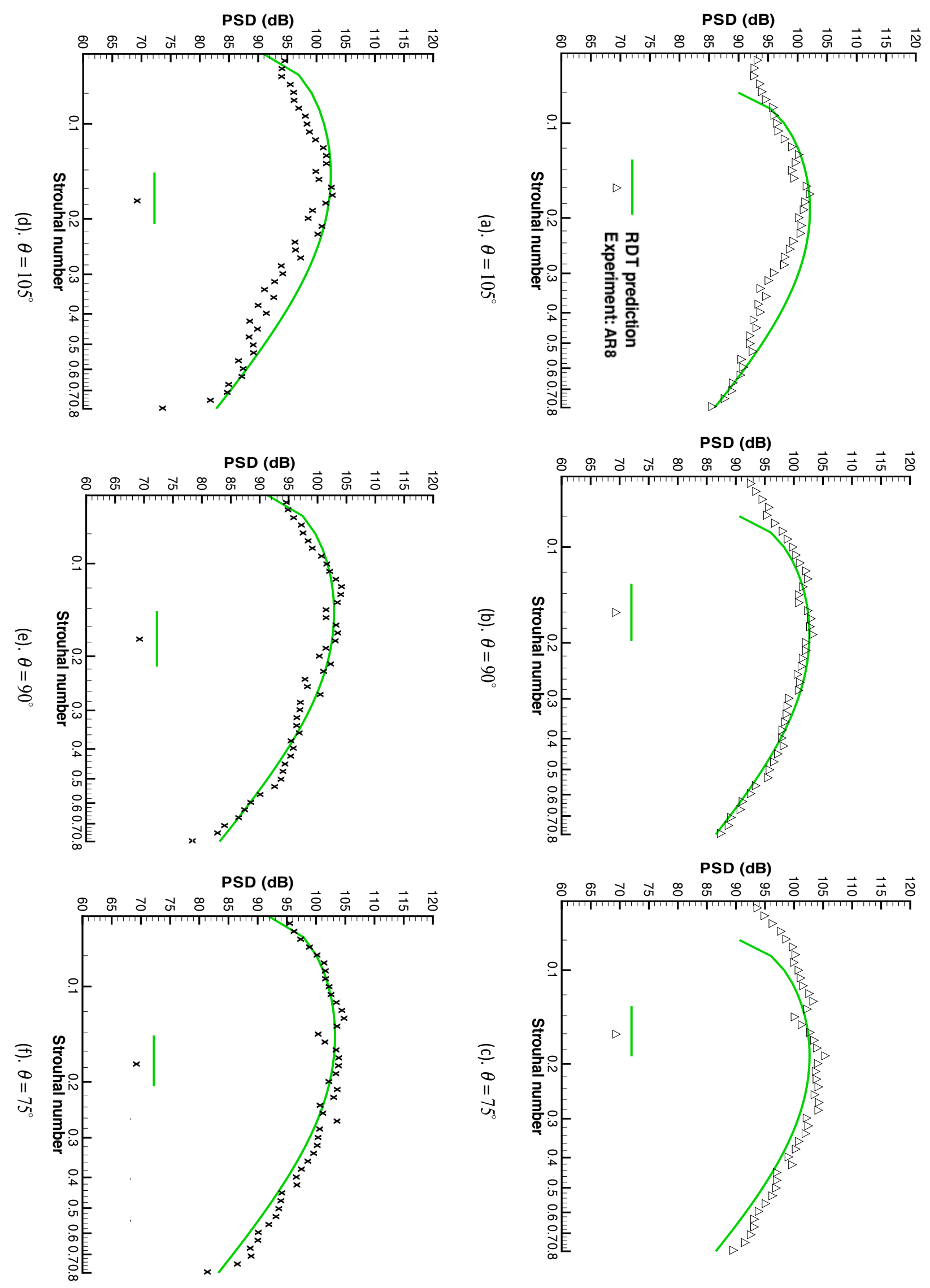

Fig. 21 RANS-based RDT prediction for $M a=0.9$ and $A R=8$ compared to noise data [3] at $\theta=\left(105^{\circ}, 90^{\circ}, 75^{\circ}\right)$ and $\psi=-90^{\circ}$. Trailing-egde location is $x_{d} / D_{J}=5.7$ and $y_{d} / D_{J}=0.25$ (figs. a-c) \& $y_{d} / D_{J}=0.4$ (figs. d-f). 
Note that the spectral range over which noise amplification occurs is greater for these cases, occurring up to $S t \sim 0.8$ for $\theta=90^{\circ}$. The predictions in Fig. 21 do rapidly decay as $\omega \rightarrow 0$ presumably because the de-correlation parameters $\left(a_{1}, a_{2}\right)$ in Eq. (15) are perhaps too large at these very low frequencies for these particular jet-plate configurations (the predictions in Figs. $20 c \& d$ also suffer from the same issue). However, our aim in Figs. $20 \& 21$ was to test the robustness of the model (i.e. how close the predictions to data when the de-correlation parameters are kept fixed). Hence, in all cases, the $\left(a_{1}, a_{2}\right)$ parameters and are identical to that shown in table 1 , with the exception of the symmetry parameter, $b_{0}$, which was kept fixed at $b_{0} / y_{d}=0.2$ for cases shown in Fig. 21. The predictions are therefore still encouraging. This is particularly true for $y_{d} / D_{J}=0.25$ case at $\theta=90^{\circ}$.

The results in Figs. 21 address the concerns raised by Bridges ([3], p. 2), by showing that the RDT-based jet-surface interaction noise predictions do compare favorably with the closely positioned plate conditions (at least on the shielded side); that is where the plate trailing edge is very near to the the nozzle lower lip line relative to $D_{J}$. This is not suprising because the experimental data itself indicates a similar spectral shape (albeit with larger frequency range of amplification) to the cases considered earlier where cases in which the trailing edge was further away.

\section{DISCUSSION AND CONCLUSIONS}

\subsection{Applicability of RDT model}

The basic assumption of RDT is that the interaction time of the turbulent eddies is short compared to their nonlinear interaction (eddy 'turn-over') time, $\tau_{\text {int }} \ll l_{\infty} /|\boldsymbol{u}|$ (where $l_{\infty}$ is the upstream turbulence spatial scale (p. 40 of [33] \& [34]), which means that the latter unsteadiness (which produces what we refer to as the 'jet noise') is being neglected. However, the purely convected gust, which is taken as the input disturbance to the RDT (see Eq. (6)), is much larger than the nonlinear sources of jet noise during the time over which the interactions take place. As we have shown in this paper, the RDT model predicts the leading-order spectral behaviour of the edge-generated noise and also provides predictions that are in reasonably good agreement with data over the nozzle aspect ratios we have considered ( $A R 4 \& A R 8$ ) when the RANS mean flow quantities are used and a model representative of the upstream turbulence near the trailing edge is tuned appropriately.

The model does not, however, predict the oscillations that are present in the data at low frequencies. These oscillations are believed to be due to interference between direct and edge-generated sound (see [46] \& [47]). In our case, the purely convected disturbance in a streamwise-homogeneous flow (the gust solution, Eq. (6)) does not produce direct acoustic radiation at subsonic speeds. However, direct acoustic radiation (i.e. the 'jet noise') is produced by the non-purely convected flow disturbances at $O(1)$ acoustic Mach numbers. 
The subsequent interference of the jet noise with the JSI noise is most likely responsible for the oscillations seen in the data.

Since the total acoustic pressure in the far-field as $|\boldsymbol{x}| \rightarrow \infty$ is given by the sum, $p^{s}+p^{\prime}$, where $p^{s}(\boldsymbol{x}, t)$ is the edge-scattered interaction noise we have considered in this paper and $p^{\prime}(\boldsymbol{x}, t)$ is the jet noise, given, for example, by Eq. (3.4) in Goldstein \& Leib [74] with the fourth component of the Greek suffixes equal to zero for isothermal jet, the far-field pressure auto-covariance, $\overline{p(\boldsymbol{x}, t) p(\boldsymbol{x}, t+\tau)}$ (where the overbar denotes time average and $p=p^{s}+p^{\prime}$ ) will be given by the sum of terms of the type shown in Table 2 below. Here, $T_{i j}^{\prime}(\boldsymbol{y}, \tau)$ is the purely fluctuating Reynolds stress.

\begin{tabular}{|c|c|c|}
\hline $\begin{array}{c}\text { Term in acoustic } \\
\text { spectrum formula }\end{array}$ & $\begin{array}{c}\text { Statistical turbulence quantity for the sound } \\
\text { source }\end{array}$ & $\begin{array}{c}\text { Order of magnitude, } \\
\alpha \equiv|\boldsymbol{u}| / U_{J} \ll O(1)\end{array}$ \\
\hline JSI noise & $\begin{array}{c}\text { Spectrum of } \tilde{\omega}_{c^{\prime}} \\
S\left(y_{2}, \tilde{y}_{2} ; k_{3}, \omega ; y_{d}\right) .\end{array}$ & $O\left(\alpha^{2}\right)$ \\
\hline JSI/jet noise & $\begin{array}{c}\text { Gust-Reynolds stress co-variance tensor, } \\
\tilde{R}_{i j}\left(\boldsymbol{y}, \eta, \tau_{0}\right)=\end{array}$ & $O\left(\alpha^{3}\right)$ \\
\hline coupling & $\frac{\tilde{\omega}_{c}\left(\tau-y_{1} / U\left(\boldsymbol{y}_{T}\right), \boldsymbol{y}_{T}\right) T_{i j}^{\prime}\left(\boldsymbol{y}+\eta, \tau+\tau_{0}\right)}{R}$ & \\
\hline Jet noise & Reynolds stress auto-covariance, \\
& $R_{i j k l}\left(\boldsymbol{y}, \eta, \tau_{0}\right)=\overline{T_{i j}^{\prime}(\boldsymbol{y}, \tau) T_{k l}^{\prime}\left(\boldsymbol{y}+\eta, \tau+\tau_{0}\right)}$ & $O\left(\alpha^{4}\right)$ \\
\hline
\end{tabular}

\section{Table 2 - Terms in the acoustic spectrum formula}

If interference effects are associated with the JSI/jet noise coupling term, its magnitude will depend, at $O(1)$ frequencies, on the individual magnitudes and phases of both the edgescattered \& jet noise. Since Goldstein ([23] p. 611 \& Eq. 2.45) indicates that $\tilde{\omega}_{c}$ will be nearly equal to the vorticity in low speed flows, the co-variance tensor, $\tilde{R}_{i j}\left(\boldsymbol{y}, \eta, \tau_{0}\right)$, must be a positive semi-definite function of its arguments. Therefore, the interference effects will be important at low frequencies where the JSI noise is greatest and be more oscillatory at high subsonic acoustic Mach numbers where jet noise is more efficient. In Fig. 1 in Brown [1], the oscillations occur more at lower than at higher frequencies (i.e. $S t>0.5$ ) where they are of the same order as the oscillations in the jet noise itself. This conclusion is the same whether the observation point is above or below the plate and also evident in the rectangular jet/plate experiments. In Fig. 22a we show the change in PSD (dB) for $\theta=90^{\circ}$ relative to the 
the jet noise from experimental data for three closely-positioned plates for which $y_{d} / D_{J}=(0,0.25,0.4)$. Since the edge-scattered noise is much more efficient at these transverse locations (with streamwise location fixed at $x_{d} / D_{J}=5.7$ ), the interference effects owing to the coupling term are relatively small. Similarly, in Fig. 22b, we shows the same jet at locations further away, $y_{d} / D_{J}=(1.7,2,2.5)$, where the jet noise dominates. Here again the oscillations are largely insignificant compared to the uncertainty in the data itself. (JSI noise occurs up to $S t \approx 0.2$ for $\left.y_{d} / D_{J}=2.5\right)$. It is only, therefore, at the intermediate trailing edge locations (as in Figs. 15 \& 16) where the interaction between the jet noise and edgescattered noise is important such that oscillations become significant.

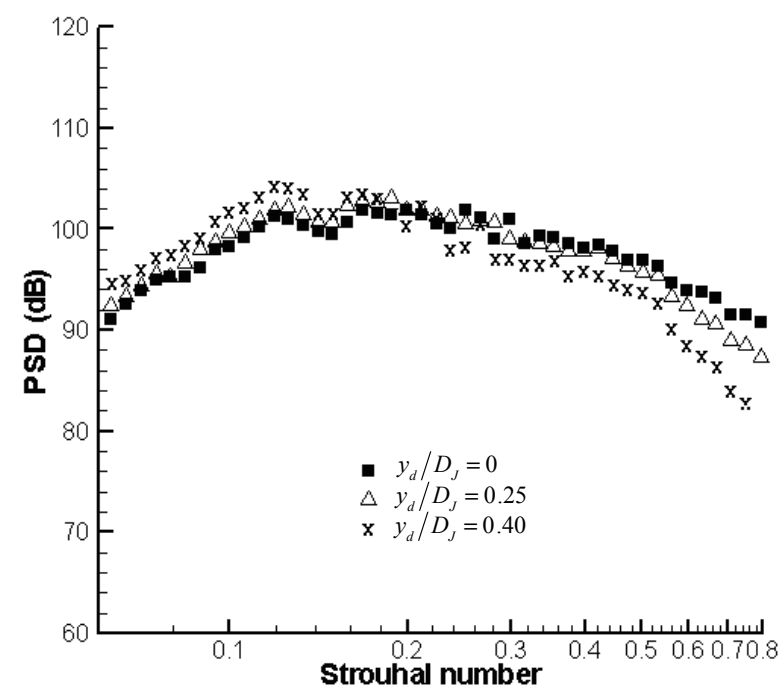

(a). Close-plate locations (edge-noise dominates)

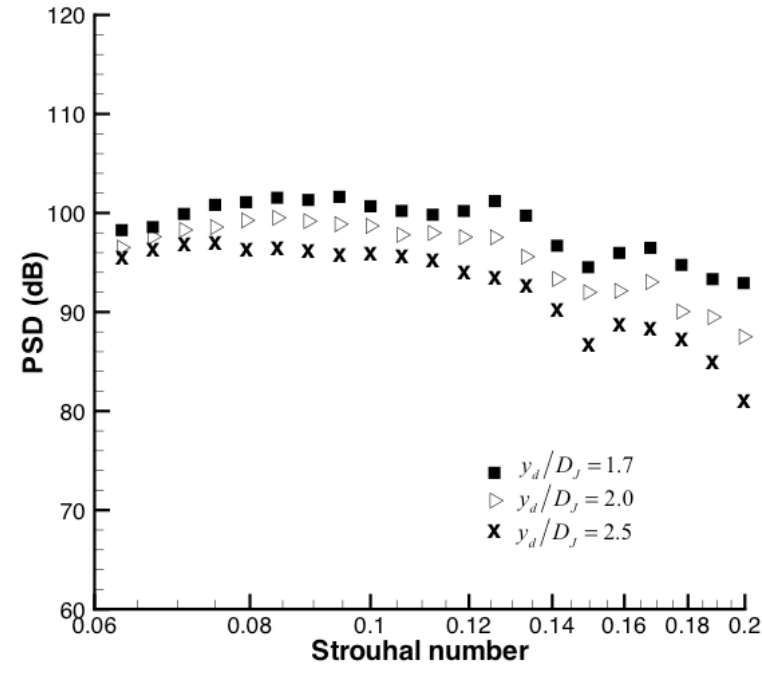

(b). Further-away (jet noise dominates)

Fig. 22- Experimental data trends for $M a=0.9$ and nozzle aspect ratio, $A R 8$ at $\theta=90^{\circ}$ [3] with plate at

$$
x_{d} / D_{J}=5.7
$$

This explanation is consistent with numerical simulations. The predictions of Paliath \& Premasuthan [52], for the same cases that we use in this paper were based on numerical simulations using LES and Ffowcs-Williams Hawkings (FFW-H) equation without the quadrupole term. The low-frequency sound they calculated did not show any noticeable sign of oscillation. See right-hand side figures in Paliath \& Premasuthan's Figs. 6-9 and also Figs. 7b \& 8c in Semiletov et al. [75].

Moreover, in a series of related papers Wolf \& Lele [76], Yu et al. [77], Wolf et al. [78] \& [79] and [18] found that in the absence of any linear/non-linear interaction, the predicted polar directivity is cardioid shaped at low frequencies. Any departure from the cardioid symmetry in the directivity pattern then appears in either as asymmetry (about the jet centerline for the polar directivity) or in the appearance of multiple lobes. These effects are much more pronounced in simulations where there is a high degree of non-linearity (i.e. turbulence) due to 
the wake of a cylinder placed near the trailing edge. Compare, for example, Figs. $5 b$ \& $9 b$ in Wolf \& Lele [76] in which polar directivity plots are shown for the unsteady flow around a cylinder \& NACA-0012 airfoil plus cylinder at the vortex shedding frequency respectively. In their latter (Fig. 9b) the quadrupole appears to cause more asymmetry in the directivity pattern than the in Fig. (5b). Although, owing to the low Mach number of simulation ( $M a=0.3)$, the dipole-quadrupole interaction effects shown here have a small effect relative to the maximum SPL value the directivity lobe attains. The Strouhal number of these simulations, based on the cylinder diameter, is low (at 0.19 , see p. 2281 of [76]). The quadrupole interaction was further investigated in [78] who found that much richer asymmetry in directivity patterns arises when the acoustic Mach number is higher. For example, in Fig. 32 in [78], the amplification associated with quadrupole term in the FFW-H equation is about $10 \mathrm{~dB}$ (p. 531) at medium and high frequencies when the Mach number is high (upto 0.4 in their simulation). This effect is also observed in the formation of asymmetric lobes for certain polar observation angles at midfrequencies (Fig 32b) when quadrupoles are included compared to the dipole-only calculation. The Wolf \& Lele results would, therefore, logically suggest that the asymetries in the directivity pattern associated with the quadrupole in the FFW-H formulation would appear at low frequencies when the Mach number is high (i.e. $>0.5$ ) because the quadrupole term is much more efficient at these speeds. Note, however, that although the spectra were not shown in the simulations, it is expected that asymmetry in directivity pattern would result in oscillation in spectra (since our calculations have shown the reverse - i.e. that a symmetric directivity pattern does not result in any spectral oscillations in the predicted sound).

Further work by Wolf et al. [79] has shown that quadrupole sources have a significant impact on amplification and/or asymetry of directivity patterns for wake interactions within an airframe configurations (i.e. airfoil plus cylinder). In all of these papers, it was found that the directivity computed by Direct Numerical Simulation (DNS) was re-produced using the FFW-H equation only with quadrupole term included. The authors note (on pp. 12-13) that their results indicate that, "even at low frequencies, quadrupole sources are of paramount importance for the total noise prediction of a moderate Mach number flow with wake interaction".

Our analysis, on the other hand, considers edge-scattered noise only. We believe that this source dominates at low frequencies and makes up most - if not all - of the jet-surface interaction noise bar interference effects associated with coupling term in Table 2. Note that an essentially similar argument is made when Amiet's approach is used. For example, Sandberg \& Sandham's first principles application of Amiet's theory (Eq. 2 in [80]) neglects the volume quadrupole term when using Goldstein's form of Lighthill's acoustic analogy (ch. 4 of [58]). This is consistent with retaining the linear part of the unsteady flow as done using RDT. As expected, Lawrence et al.'s ([17], Fig. 14) application of Amiet's model, also captures the gross amplification but not the detailed oscillation-which is consistent with our model.

\subsection{Concluding remarks}

In this paper we have extended the Goldstein-Afsar-Leib ([24], GAL) jet-surface interaction model to include a negative dip (or, de-correlation) in the upstream turbulence, Eq. (14), using the more general turbulence model defined by Eq. (15) \& (16) for the transverse twopoint correlation function. This model exhibits properties of type shown in Fig. 2 (the auto- 
correlation of Eq. (14)). We have shown, using simple asymptotic arguments and numerical analysis, that the presence of a de-correlation region (i.e. taking $\left(a_{1}, a_{2}\right) \neq 0$ in Eq. (14)) directly affects the low-frequency algebraic decay of the jet-surface interaction noise spectrum. This decay, often termed the low frequency 'roll-off', must be $O\left(\omega^{2}\right)$ for the acoustic field to be consistent with a purely dipole-like acoustic source. A finite decorrelation is required in our model for the roll-off to have a pre-factor that scales as $O\left(\omega^{2}\right)$ at very low frequencies. The jet-surface interaction model Eq. (17), based on the nonhomogeneous rapid-distortion theory (RDT), allows the turbulence conditions to be specified as its upstream boundary condition through an approximate algebraic relation Eq. (10) that is a function of the two-point time-delayed correlation Eq. (14) of the stationary random function $\rho v_{\perp}^{\prime(0)}(\boldsymbol{x}, t) \quad$ (transverse momentum fluctuation), which Deissler [39] and Tennekes \& Lumley [40] explain, must become negative with increase in spatial separation and/or time delay in a time-stationary turbulence field. In constrast, the original GAL jet-surface interaction model (Eq. 6.50 in that paper) had $\left(a_{1}, a_{2}\right)=0$ in Eq. (15) and thereby produced a spectrum that tends to $O(1)$ at very low frequencies (less than peak), which is at worst 10 $\mathrm{dB}$ greater than experiment (see Fig. $5 \mathrm{~b}$ ).

The RDT predictions also provide a first estimate of the interaction noise from more nonplanar lower-aspect ratio rectangular jet nozzles (Figs. 7-9). In addtion, we have implemented a RANS-based RDT prediction method in which (as in acoustic analogy approaches) we determine the changes in length scales and amplitude of the transverse velocity correlation for different nozzle aspect ratio and acoustic Mach numbers using the local the turbulent kinetic energy and rate of energy dissipation values obtained from these solutions. This approach generally gives much closer agreement with data for AR8 (Figs.1517 ) and AR4 (4:1 Aspect ratio) nozzle (see Fig. 18). Calculations at different plate locations (Figs. 19 \& 20) were also performed to test the robustness of the model (by keeping the decorrelation parameters fixed). They show good agreement at low frequencies and in the case of Fig. $20 \mathrm{~d}$ even at higher frequencies relative to the peak jet-surface interaction noise. Moreover, we have shown that even for the closely-alligned flat plates (Figs. 21 \& 22) that first-estimate predictions can be obtained for the peak interaction noise by, as previously, keeping the $a_{0,1,2}$ parameters in Eq. (15) fixed. It is expected, however, that with further experimentation of $a_{0,1,2}$, as well as the length scales in (16), the predictions could be made to agree better over a much wider range of both aspect ratio and acoustic Mach number.

Though the agreement is closer to the data than GAL's results, it appears that in some of the trailing-edge locations we have considered (e.g. Fig. 7), the data decays faster than $O\left(\omega^{2}\right)$ at very low Strouhal numbers. The increased rate of roll-off is apparent at higher 
Mach numbers and when $y_{d} / D_{J} \sim 1$ (when $x_{d} / D_{J}=5.7$ ) for the $90^{\circ}$ spectrum (see Figs. 7 $\& 15)$ and cannot be explained by the purely dipole-like model we have constructed here.

Any empiricism introduced by tuning the scales from the RANS calculation could be eliminated by using experimental or LES data on turbulence (as, for example in [[82]). Alternatively, a computational optimization approach ([81]-[83]), where the parameters are optimized numerically could be used. Interestingly, however, the model formula Eq. (17) appeared to most sensitive only to the parameters that determine the de-correlation region and the spanwise body length scale, and only these properties were changed between predicitons for different nozzle aspect ratio ratios. These can, in some sense, be justified theoretically to model the effect of spanwise inhomogeneity in the smaller ascpet ratio cases. We hope that - as in the development of the acoustic analogy approach - this work will inspire efforts to analyze the structure of the turbulence near the trailing edge (e.g. to get a better understanding of the symmetry parameter $b_{0}$ in Eq. (11)) and, in the context of the model, attempts to eliminate any empirical tuning by using Large-Eddy Simulations (LES). We note that, particularly for the smaller aspect ratio jets, that the spanwise coherence effects sould also impact the low-frequency roll-off of the jet-surface interaction noise spectrum and would need to incorported into the model.

Appendix A: construction of a turbulence model with $\left(a_{1}, a_{2}\right) \neq 0$ in Eq. (14) Goldstein, Afsar \& Leib [24][21] (GAL) derived a model for the source function $S\left(y_{2}, \tilde{y}_{2} ; k_{3}^{(s)}, \omega, y_{d}\right)$, Eq. (10), that depends on the upstream turbulence through Eqs. (8) and (9). We suppose, therefore, that the experimentally measureable transverse velocity spectrum, $F_{\perp}\left(x_{2}, \tilde{x}_{2} \mid y_{2}, \tilde{y}_{2}, \omega, k_{3}\right)$, at the transverse location $\left(x_{2}, \tilde{x}_{2}\right)=y_{d}$ (in co-ordinate $\boldsymbol{x}$ ) is given by streamwise Fourier transforms of the function Eq.(15) where $a_{n}=\left(a_{0}, a_{1}, a_{2}, \ldots, a_{n} \ldots a_{N}\right)$ are weighting coefficients that, like the decay function $X\left(\eta_{1},|\eta| \tau ; \alpha\right)$, could be obtained from experiments or LES/DNS. As in Leib \& Goldstein [38] the decay function is taken as

$$
X\left(\eta_{1},|\eta|, \tau ; \alpha\right)=\sqrt{\left(\eta_{1} / l_{1}\right)^{2}+\left[\left(\eta_{1}-U_{c} \tau\right) / l_{0}\right]^{2}+\left(\eta_{3} / l_{3}\right)^{2}+\alpha^{2}}
$$

where $\eta_{1} \equiv \tilde{x}_{1}-x_{1}$ and the amplitude function $\Psi\left(\bar{x}_{1}\right)$ is a function of symmetric streamwise location, $\bar{x}_{1} \equiv\left(x_{1}+\tilde{x}_{1}\right) / 2$.

\section{A.1. Computation of Fourier transforms}

Inserting the amplitude function $\Psi\left(\bar{x}_{1}\right)$ (defined below Eq. (16)) into Eq. (15) we find that $f_{\perp}\left(x_{2}, \tilde{x}_{2} \mid k_{1}, \tilde{k}_{1}, \eta_{3}, \tau ; \alpha\right)$ at location $\left(x_{2}, \tilde{x}_{2}\right)=y_{d}$ is given by (using Eq. (9)) 


$$
f_{\perp}\left(y_{d}, y_{d} \mid k_{1}, \tilde{k}_{1}, \eta_{3}, \tau ; \alpha\right)=L_{3} \frac{\Psi_{0}}{(2 \pi)^{2}} \int_{-\infty}^{\infty} \int_{-\infty}^{\infty} e^{-i\left(x_{1} k_{1}-\tilde{x}_{1} \tilde{k}_{1}\right)} e^{\alpha-\left(\bar{x}_{1} / L_{1}\right)^{2}} D\left(\tau, \eta_{1}, \eta_{3}\right) d x_{1} d \tilde{x}_{1}
$$

where $D\left(\tau, \eta_{1}, \eta_{3}\right)$ is the sum of terms in Eq. (15).

Changing variables in Eq. (A.2) to $\eta_{1} \equiv \tilde{x}_{1}-x_{1}$ so that $\bar{x}_{1} \equiv x_{1}+\eta_{1} / 2$ and evaluating the Gaussian integral shows that $f_{\perp}\left(y_{d}, y_{d} \mid k_{1}, \tilde{k}_{1}, \eta_{3}, \tau ; \alpha\right)$ is given by

$$
f_{\perp}\left(y_{d}, y_{d} \mid k_{1}, \tilde{k}_{1}, \eta_{3}, \tau ; \alpha\right)=\frac{L_{1} L_{3} \Psi_{0}}{4 \pi^{3 / 2}} e^{\alpha-\left[\left(k_{1}-\tilde{k}_{1}\right) L_{1} / 2\right]^{2}} \int_{-\infty}^{\infty} e^{i \overline{k_{1}} \eta_{1}} D\left(\tau, \eta_{1}, \eta_{3}\right) d \eta_{1}
$$

where $\bar{k}_{1}$ the is the average streamwise wavenumber, $\bar{k}_{1}=\left(k_{1}+\tilde{k}_{1}\right) / 2$. Now taking Fourier transforms of Eq. (A.2) in time delay, $\tau$, and substituting for $D\left(\tau, \eta_{1}, \eta_{3}\right)$ from Eq. (15) gives:

$$
\begin{aligned}
& \frac{1}{2 \pi} \int_{-\infty}^{\infty} e^{-i \omega \tau} f_{\perp}\left(y_{d}, y_{d} \mid k_{1}, \tilde{k}_{1}, \eta_{3}, \tau ; \alpha\right) d \tau \\
& = \\
& \frac{L_{1} L_{3} \Psi_{0}}{2 \pi^{1 / 2}} e^{\alpha-\left[\left(k_{1}-\tilde{k}_{1}\right) L_{1} / 2\right]^{2}} \frac{1}{(2 \pi)^{2}} \int_{-\infty}^{\infty} \int_{-\infty}^{\infty} e^{i \bar{k}_{1} \eta_{1}} e^{-i \omega \tau}\left[a_{0}+a_{1} \tau \frac{\partial}{\partial \tau}+a_{2} \eta_{1} \frac{\partial}{\partial \eta_{1}}+\ldots\right] e^{-X\left(\eta_{1},|\eta| \tau\right)} d \eta_{1} d \tau
\end{aligned}
$$

Re-writing the $\left(\tau, \eta_{1}\right)$ pre-factors as derivatives in $\left(\omega, \bar{k}_{1}\right)$ respectively and integrating the remaining terms in the square brackets by parts gives the algebraic result

$$
\begin{aligned}
& \frac{1}{2 \pi} \int_{-\infty}^{\infty} e^{-i \omega \tau} f_{\perp}\left(y_{d}, y_{d} \mid k_{1}, \tilde{k}_{1}, \eta_{3}, \tau ; \alpha\right) d \tau \\
& =\frac{L_{1} L_{3} \Psi_{0}}{2 \pi^{1 / 2}} e^{\alpha-\left[\left(k_{1}-\tilde{k}_{1}\right) L_{1} / 2\right]^{2}}\left[\left(a_{0}-a_{1}-a_{2}\right)-a_{1} \omega \frac{\partial}{\partial \omega}-a_{2} \bar{k}_{1} \frac{\partial}{\partial \bar{k}_{1}}+\ldots\right] \Phi\left(\bar{k}_{1}, \omega, \eta_{3}\right)
\end{aligned}
$$

since the decay function of the turbulence, $e^{-X\left(\eta_{1}, \eta, \tau\right)}$ is bounded in $\left(\eta_{1},|\boldsymbol{\eta}|, \tau\right)$ and where $\Phi\left(\bar{k}_{1}, \omega, \eta_{3} ; \alpha\right)$ now denotes the spectrum of the leading order decay function:

$$
\Phi\left(\bar{k}_{1}, \omega, \eta_{3} ; \alpha\right)=\frac{1}{(2 \pi)^{2}} \int_{-\infty}^{\infty} \int_{-\infty}^{\infty} e^{i \bar{k}_{1} \eta_{1}} e^{-i \omega \tau} e^{-X\left(\eta_{1}, \mid \eta, \tau\right)} d \eta_{1} d \tau
$$


Introducing non-dimensional independent variables: $\tilde{\tau}=U_{c} \tau / l_{0}, \tilde{\eta}_{1}=\eta_{1} / l_{1}, \tilde{\eta}_{2}=\eta_{2} / l_{2}$ and $\tilde{\eta}_{3}=\eta_{3} / l_{3}$ (Leib and Goldstein[38], Eqs. 25 \& 26) allows the inner Fourier transform in $\tilde{\eta}_{1}$ to be evaluated using the tabulated results in Campbell \& Foster [84] (result 867 on p.111):

$$
\begin{aligned}
& \Phi\left(\bar{k}_{1}, \omega, \eta_{3} ; \alpha\right)=\left(\frac{1}{\pi}\right) \frac{l_{0} l_{1}}{U_{c}} \frac{1}{\left[\left(\bar{k}_{1}-\omega / U_{c}\right)^{2} l_{1}^{2}+1\right]^{1 / 2}} \times \\
& \frac{1}{2 \pi} \int_{-\infty}^{\infty} e^{i\left(\omega l_{0} / U_{c}\right) \tilde{\xi}}\left[\tilde{\eta}_{3}^{2}+\alpha^{2}+\tilde{\xi}^{2}\right]^{1 / 2} K_{1}\left[\sqrt{\left[\tilde{\eta}_{3}^{2}+\alpha^{2}+\xi^{2}\right]\left[\left(\bar{k}_{1}-\omega / U_{c}\right)^{2} l_{1}^{2}+1\right]}\right] d \tilde{\xi}
\end{aligned}
$$

where $\tilde{\xi}=\tilde{\eta}_{1} l_{1} / l_{0}-\tilde{\tau}$ and $K_{1}[\ldots]$ is the modified Bessel function of the second kind with indicated arguments. Taking Fourier transforms in $\eta_{3}$ :

$$
\begin{aligned}
\hat{\Phi}\left(\bar{k}_{1}, \omega, k_{3} ; \alpha\right) & =\frac{1}{2 \pi} \int_{-\infty}^{\infty} e^{i k_{3} \eta_{3}} \Phi\left(\bar{k}_{1}, \omega, \eta_{3} ; \alpha\right) d \eta_{3}=\frac{l_{3}}{2 \pi} \int_{-\infty}^{\infty} e^{i k_{3} l_{3} \tilde{\eta}_{3}} \Phi\left(\bar{k}_{1}, \omega, \tilde{\eta}_{3} ; \alpha\right) d \tilde{\eta}_{3} \\
& =\left(\frac{1}{\pi}\right) \frac{l_{0} l_{1} l_{3}}{U_{c}} \frac{1}{\left[\left(\bar{k}_{1}-\omega / U_{c}\right)^{2} l_{1}^{2}+1\right]^{1 / 2}} \times \hat{\Theta}\left(\omega, k_{3} ; \alpha\right)
\end{aligned}
$$

where $\hat{\Theta}\left(\omega, k_{3} ; \alpha\right)$ is now the spanwise Fourier transform:

$$
\begin{aligned}
& \hat{\Theta}\left(\omega, k_{3} ; \alpha\right) \\
& = \\
& \frac{1}{(2 \pi)^{2}} \int_{-\infty}^{\infty} \int_{-\infty}^{\infty} e^{i k_{3} l \tilde{\eta}_{3}} e^{i\left(\omega l_{0} / U_{c}\right)}\left(\tilde{\eta}_{3}^{2}+\alpha^{2}+\tilde{\xi}^{2}\right)^{1 / 2} K_{1}\left\{\sqrt{\left[\tilde{\eta}_{3}^{2}+\alpha^{2}+\xi^{2}\right]\left[\left(\bar{k}_{1}-\omega / U_{c}\right)^{2} l_{1}^{2}+1\right]}\right\} d \tilde{\eta}_{3} d \tilde{\xi}
\end{aligned}
$$

that can be evaluated using Fourier transform tables ([84] ,result 917.8 on p. 125) to give

$$
\begin{aligned}
& \hat{\Theta}\left(\omega, k_{3} ; \alpha\right) \\
& =\left(\left(\overline{k_{1}}-\omega / U_{c}\right)^{2} l_{1}^{2}+1\right)^{1 / 2} \frac{1}{2 \chi^{3 / 2}} \frac{1}{2 \pi} \int_{-\infty}^{\infty} e^{i\left(\omega l_{0} / U_{c}\right) \xi}\left(1+\left(\alpha^{2}+\tilde{\xi}^{2}\right)^{1 / 2} \chi^{1 / 2}\right) e^{-\chi^{1 / 2}\left(\alpha^{2}+\tilde{\xi}^{2}\right)^{1 / 2}} d \tilde{\xi}
\end{aligned}
$$


where

$$
\chi=\left(k_{3} l_{3}\right)^{2}+\left(\bar{k}_{1}-\omega / U_{c}\right)^{2} l_{1}^{2}+1
$$

Differentiating under the integral sign allows further simplification of Eq. (A.10) as

$$
\hat{\Theta}\left(\omega, k_{3} ; \alpha\right)=-\left[\left(\bar{k}_{1}-\omega / U_{c}\right)^{2} l_{1}^{2}+1\right]^{1 / 2} \frac{\partial}{\partial \chi} \frac{1}{\chi^{1 / 2}} \frac{1}{2 \pi} \int_{-\infty}^{\infty} e^{i\left(\omega l_{0} / U_{c}\right) \xi-\chi^{1 / 2}\left(\alpha^{2}+\xi^{2}\right)^{1 / 2}} d \tilde{\xi}
$$

which, again, by Campbell \& Foster ([84], p.111, result 867) can be evaluated:

$$
\hat{\Theta}\left(\omega, k_{3} ; \alpha\right)=-\left(\left(\bar{k}_{1}-\omega / U_{c}\right)^{2} l_{1}^{2}+1\right)^{1 / 2} \frac{1}{\pi} \frac{\partial}{\partial \chi}\left\{\frac{\alpha K_{1}\left\{\alpha\left(\left(\omega l_{0} / U_{c}\right)^{2}+\chi\right)^{1 / 2}\right\}}{\left(\left(\omega l_{0} / U_{c}\right)^{2}+\chi\right)^{1 / 2}}\right\} .
$$

Hence the spanwise Fourier transform of the leading order spectral function, Eq. (A.10), is the reasonably simple result

$$
\hat{\Phi}\left(\bar{k}_{1}, \omega, k_{3} ; \alpha\right)=\left(\frac{1}{\pi^{2}}\right) \frac{l_{0} l_{1} l_{3}}{U_{c}} \hat{H}\left(\chi\left(\bar{k}_{1}, \omega, k_{3}\right), \omega ; \alpha\right)
$$

with spectral function,

$$
\hat{H}\left(\chi\left(\bar{k}_{1}, k_{3}\right), \omega ; \alpha\right)=-\frac{\partial}{\partial \chi}\left\{\frac{\alpha K_{1}\left[\alpha\left(\left(\omega l_{0} / U_{c}\right)^{2}+\chi\right)^{1 / 2}\right]}{\left(\left(\omega l_{0} / U_{c}\right)^{2}+\chi\right)^{1 / 2}}\right\}
$$

that depends on $\left(\bar{k}_{1}, k_{3}\right)$ only through $\chi$ as defined by Eq. (A.11). But since Abramowitz \& Stegun ([85], p. 375) show $z K_{1}(z) \rightarrow 1$, as $z \rightarrow 0$, as $\alpha \rightarrow 0$ the spanwise Fourier transform of the spectral function $\hat{H}\left(\chi\left(\bar{k}_{1}, k_{3}\right), \omega, \alpha\right)$ reduces to:

$$
\tilde{H}\left(\chi\left(\bar{k}_{1}, k_{3}\right), \omega\right)=-\frac{\partial}{\partial \chi}\left\{\frac{1}{\left(\omega l_{0} / U_{c}\right)^{2}+\chi}\right\}=\frac{1}{\left(\left(\omega l_{0} / U_{c}\right)^{2}+\chi\right)^{2}}
$$

The spanwise Fourier transform, Eq. (A.14), of the leading order spectral function $\hat{\Phi}\left(\bar{k}_{1}, \omega, k_{3}\right)$ is algebraically very simple in the case where $\alpha \rightarrow 0$ (it also corresponds to the a correlation function possessing a cusp on the axis-which has been observed for lateral correlation function of the type (14) ). We therefore focus our attention here. 


\section{A.2. Generalization of GAL model}

Inserting the spanwise Fourier transform of Eq. (A.5) into Eq. (8) gives the complete spectral function of the transverse momentum correlation function:

$$
\begin{aligned}
& \hat{F}_{\perp}\left(x_{2}, \tilde{x}_{2}, k_{1}, \tilde{k}_{1} \mid y_{2}, \tilde{y}_{2}, \omega, k_{3}\right) \\
& =\frac{L_{1} L_{3} \Psi_{0}}{2 \pi^{1 / 2}} e^{-\left[\left(k_{1}-\tilde{k}_{1}\right) L_{1} / 2\right]^{2}}\left[\left(a_{0}-a_{1}-a_{2}\right)-a_{1} \omega \frac{\partial}{\partial \omega}-a_{2} \bar{k}_{1} \frac{\partial}{\partial \bar{k}_{1}}+\ldots\right] \hat{\Phi}\left(\bar{k}_{1}, \omega, k_{3}\right)
\end{aligned}
$$

After substituting Eq. (A.14) gives

$$
\hat{F}_{\perp}\left(x_{2}, \tilde{x}_{2}, k_{1}, \tilde{k}_{1} \mid y_{2}, \tilde{y}_{2}, \omega, k_{3}\right)=\frac{L_{1} L_{3} \Psi_{0}}{2 \pi^{1 / 2}} e^{-\left[\left(k_{1}-\tilde{k}_{1}\right) L_{1} / 2\right]^{2}}\left(\frac{1}{\pi^{2}}\right) \frac{l_{0} l_{1} l_{3}}{U_{c}} \hat{\Pi}\left(\bar{k}_{1}, \omega, k_{3}, 0\right)
$$

in which coefficient,

$$
\tilde{\Pi}\left(\bar{k}_{1}, \omega, k_{3}\right)=\left[\left(a_{0}-a_{1}-a_{2}\right)-a_{1} \omega \frac{\partial}{\partial \omega}-a_{2} \bar{k}_{1} \frac{\partial}{\partial \bar{k}_{1}}+\ldots\right] \hat{H}\left(\chi\left(\bar{k}_{1}, \omega, k_{3}\right), \omega, \alpha\right)
$$

reduces to Eq. (19) after differentiating Eq. (A.11) and taking hydrodynamic wavenumber limit, $\left.k_{1} \rightarrow \omega / U\left(y_{2}\right) \quad \& \quad \tilde{k}_{1} \rightarrow \omega / U\left(\tilde{y}_{2}\right)\right)$. Hence

$$
F_{\perp}\left(x_{2}, \tilde{x}_{2} \mid y_{2}, \tilde{y}_{2}, \omega, k_{3}\right)=\lim _{k_{1} \rightarrow \omega / U\left(y_{2}\right) \tilde{k}_{1} \rightarrow \omega / U\left(\tilde{y}_{2}\right)} \hat{F}_{\perp}\left(x_{2}, \tilde{x}_{2}, k_{1}, \tilde{k}_{1} \mid y_{2}, \tilde{y}_{2}, \omega, k_{3}\right)
$$

is given by

$$
\begin{aligned}
& F_{\perp}\left(x_{2}, \tilde{x}_{2} \mid y_{2}, \tilde{y}_{2}, \omega, k_{3}\right) \\
& =\frac{L_{1} L_{3} \Psi_{0}}{2 \pi^{1 / 2}} e^{-\left[\left(\omega / U\left(y_{2}\right)-\omega / U\left(\tilde{y}_{2}\right)\right) L_{1} / 2\right]^{2}}\left(\frac{1}{\pi^{2}}\right) \frac{l_{1} l_{1} l_{3}}{U_{c}} \tilde{\Pi}\left(\left(\left[\omega / U\left(y_{2}\right)+\omega / U\left(\tilde{y}_{2}\right)\right]\right) / 2, \omega, k_{3}\right)
\end{aligned}
$$

after Eq. (A.19) is evaluated at this limit. Note that if $a_{1}=a_{2}=0$ then $\tilde{\Pi}\left(\bar{k}_{1}, \omega, k_{3}\right)$ depends on $\left(\bar{k}_{1}, \omega, k_{3}\right)$ only through $\chi\left(\bar{k}_{1}, \omega, k_{3}\right)$ and the upstream boundary condition reduces to the purely positive auto-correlation example considered by GAL (Eq. 6.48).

The integral over $\tilde{y}_{2}$ in the acoustic spectrum Eq. (12) can be performed analytically since consistent with our defintion of the gust solution being defined on a streamwise homogeneous flow (i.e. that is doubly infinite and satisfies Eq. (5) at all streamwise locations, $y_{1}$ ), the body length scale $L_{1} \rightarrow \infty$. Then since (see, e.g. Lighthill [86] p. 17) 
$L_{1} e^{-\left[L_{1}\left(k_{1}-\tilde{k}_{1}\right) / 2\right]^{2}} \rightarrow 2 \sqrt{\pi} \delta\left(k_{1}-\tilde{k}_{1}\right)$ as $\quad L_{1} \rightarrow \infty \quad$ and $\quad \omega \delta\left(k_{1}-\tilde{k}_{1}\right) / U^{2}\left(y_{2}\right)=\delta\left(U\left(y_{2}\right)-U\left(\tilde{y}_{2}\right)\right)$ (where $\left(k_{1}, \tilde{k}_{1}\right)$ are hydrodynamic wavenumbers at transverse locations $\left.\left(y_{2}, \tilde{y}_{2}\right)\right)$ the integral over $\tilde{y}_{2}$ can be evaluated by integrating over the Dirac-delta function. In order to simplify this result even further, however, GAL (p. 557) split $\tilde{\omega}_{c}\left(\tau-y_{1} / U\left(\boldsymbol{y}_{T}\right), \boldsymbol{y}_{T}\right)$ into its even and odd parts:

$$
\hat{\Omega}\left(y_{2} ; \omega, k_{3}\right)=A\left(\left(y_{2}-y_{d}\right)^{2} ; \omega, k_{3}\right)+\left(y_{2}-y_{d}\right) B\left(\left(y_{2}-y_{d}\right)^{2} ; \omega, k_{3}\right)
$$

where the spanwise-temporal Fourier transform is

$$
\hat{\Omega}\left(y_{2} ; \omega, k_{3}\right) \equiv \frac{1}{(2 \pi)^{2}} \lim _{T \rightarrow \infty} \int_{-\infty}^{\infty} \int_{-T}^{T} e^{i \omega\left(z-y_{3} k_{3} / \omega\right)} \tilde{\omega}_{c}\left(z, \boldsymbol{y}_{T}\right) d z d y_{3},
$$

and where $B=O\left(y_{d}^{-1}\right)$ in order that (A.22) remain dimensionless. We suppose that the ratio of even to odd component is $B / A=y_{d} / b_{0}=O(1)$ and fixed at a particular trailing edge location, $y_{d} / D_{J}$ with $b_{0}$ being the an additional $O(1)$ parameter. Again, we use this approximation in the present analysis to simplify the resulting formulae and since such additional complication will not necessarily alter the role the de-correlation region plays in the acoustic spectrum.

Appendix B: Approximate solution to integral Eq. (18)

Eq. (11) shows that $E\left(y_{2} ; k_{3}^{(s)}, \omega, y_{d}\right)$ expands as

$$
E\left(y_{2} ; 0, \omega\right) \equiv \frac{1}{c_{\infty}^{2}}\left(\left[U\left(y_{d}\right)-U\left(y_{2}\right)\right]+i \omega N\right)+O\left(y_{2}-y_{d}\right)
$$

since $U\left(y_{2}\right)=U\left(y_{d}\right)+O\left(y_{2}-y_{d}\right)$ and $c^{2}\left(y_{2}\right)=c_{\infty}^{2}+O\left(y_{2}-y_{d}\right)$ when $y_{2}=O\left(y_{d}\right)$, where $N\left(y_{d}\right)$, is given by

$$
N\left(y_{d}\right)=\frac{\pi c_{\infty} U_{d} b_{0}}{U^{\prime \prime} y_{d} \sqrt{c_{\infty}^{2}-U_{d}^{2}}}
$$

in the plane perpendicular to the plate where $\psi= \pm \pi / 2$ and $\beta \equiv\left(1-\sin ^{2} \theta \cos ^{2} \psi\right)^{1 / 2}=1$. The integral in Eq. (18) then expands as 


$$
\begin{aligned}
& I_{0}(M a, \theta, \pm \pi / 2)= \\
& \quad 4 \frac{c_{\infty} M a^{3} \tilde{\Pi}\left(\omega / U_{d}, \omega, 0\right)}{(1-M a \cos \theta)^{2}(1-M a)} \int_{0}^{M a} \frac{1}{\left|E\left(y_{2} ; 0, \omega\right)\right|^{2}} d M\left(y_{2}\right), \quad \text { for } y_{2}=O\left(y_{d}\right)
\end{aligned}
$$

where $M a=M\left(y_{d}\right)$ and $U_{d}=U\left(y_{d}\right)$. Inserting Eq. (B.1) into this latter integral shows:

$$
\begin{aligned}
& I_{0}(M a, \theta, \pm \pi / 2) \approx \\
& \quad 4 \frac{c_{\infty}^{4} U_{d}^{3} \tilde{\Pi}\left(\omega / U_{d}, \omega, 0\right)}{(1-M a \cos \theta)^{2}(1-M a)} \int_{0}^{U_{d}} \frac{1}{\left[U\left(y_{d}\right)-U\left(y_{2}\right)\right]^{2}+(\omega N)^{2}} d U\left(y_{2}\right), \\
& =4 \frac{c_{\infty}^{4} U_{d}^{3} \tilde{\Pi}\left(\omega / U_{d}, \omega, 0\right)}{(1-M a \cos \theta)^{2}(1-M a)} \frac{\pi}{2 \omega N}, \quad \text { for } y_{2}=O\left(y_{d}\right)
\end{aligned}
$$

The integral in Eq. (B.4) is now $O(1 / \omega)$ where $N=O(1)$. But given that Eq. (A.11) shows $\chi\left(\omega / U_{d}, 0\right) \rightarrow 1$ for $\omega \rightarrow 0$, the function, $\tilde{\Pi}\left(\omega / U_{d}, \omega, 0\right)$ (using Eq. (19)) must expand as,

$$
\tilde{\Pi}\left(\omega / U_{d}, \omega, 0\right) \sim\left\{\begin{array}{c}
1, \text { for } a_{1}=a_{2}=0 \\
\omega^{2}, \text { for } a_{0} \sim a_{1}
\end{array}\right.
$$

and, therefore, integral Eq. (18) possesses algebraic decay rates

$$
I_{0}(M a, \theta, \pm \pi / 2) \sim\left\{\begin{array}{c}
1 / \omega, \text { for } a_{1}=a_{2}=0 \\
\omega, \text { for } a_{0} \sim a_{1}
\end{array}\right.
$$

as $\omega \rightarrow 0$.

\section{Acknowledgments}

MZA would like to thank financial support from Chapman Fellowship (2013-2014) at Imperial College London, Department of Mathematics and would like to thank Professor J. T. Stuart of Imperial College for his most insightful comments. The work was also supported by the NASA Fundamental Aeronautics Program, High Speed Project. The authors would like to thank Drs. Khairul Zaman, James Bridges and Clifford Brown for providing their experimental data. 


\section{References}

[1] C.A. Brown 2012, Jet-Surface interaction test: far-field results. Proceedings of the ASME Turbo Expo 2012: Power for Land, Sea and Air GT2012, Copenhagen, Denmark,

[2] J.E. Bridges, C. A. Brown and R. F. Bozak 2014, Experiments on exhaust noise of tightly integrated propulsion systems, AIAA Paper 2014-2904.

[3] J. E. Bridges 2014, Noise from Aft Deck Exhaust Nozzles - Differences in Experimental Embodiments, AlAA Paper 2014-0876.

[4] R. P. Young, R. J. Gaeta and D. Mavris 2010, Development of a prediction method for jet installation noise: reflection/shielding, AIAA 2010-3919, $16^{\text {th }}$ AIAA/CEAS Aero-acoustics Conference.

[5] S. Powell, A. Sobester and P. Joseph 2011 Performance and noise trade-offs on a civil airliner with over-thewing engines, AIAA 2011-266, $49^{\text {th }}$ AIAA Aerospace Sciences Meeting, Orlando, Florida.

[6] N. N. Pastouchenko and C. K. W. Tam 2005, Installation effects on the flow and noise of an under-the-wing mounted dual stream jet, AIAA 2005-604, 43 ${ }^{\text {rd }}$ AIAA Aerospace Sciences Meeting, Reno, Nevada.

[7] S. Redonnet, C. Desquensnes, E. Manoha and C. Parzani 2010, Numerical Study of acoustic installation effects with a computational aeroacoustics method, AIAA Journal, Vol. 48, 2010.

[8] V. Kopiev 2015, Investigations of jet-flap interaction noise in TSAGI, $22^{\text {nd }}$ International Congress on Sound and Vibration, Florence, Italy, July 2015.

[9] M. Bondarenko, Z. Hu and X. Zhang 2012, Large-Eddy Simulation of the Interaction of a Jet with a Wing. AIAA Paper 2012-2254, $18^{\text {th }}$ AIAA/CEAS Aero-acoustics Conference, Colorado Springs, Colorado, USA.

[10] M. Perrino 2014, An Experimental Study into Pylon, Wing, and Flap Installation Effects on Jet Noise Generated by Commercial Aircraft. Ph. D. Thesis, University of Cincinnati.

[11] T. D. Scharton, B. Pinkel, J.F. Wilby. A study of trailing edge blowing as a means of reducing noise generated by the interaction of flow with a surface. NASA CR-132270.

[12] R. W. Head and M. J. Fisher 1976, Jet/surface interaction noise: - analysis of farfield low frequency augmentations of jet noise due to the presence of a solid shield. AIAA Paper 76-502, $3^{\text {rd }}$ AIAA Aeroacoustics Conference, Palo Alto California.

[13] W. Olsen and D. Boldman 1979, Trailing edge noise data with comparison to theory, AIAA Paper 79-1524.

[14] M. E. Wang 1981, Wing effect on jet noise propagation, J. Aircraft, Vol. 18, No. 4, pp. 295 - 302, 1981.

[15] I. S. Southern 1980, Exhaust noise in flight: the role of acoustic installation effects, $6^{\text {th }}$ AlAA Aeroacoustics Conference, Hartford, Connecticut. 
[16] C. J. Mead and P. J. Strange 1998, Under wing installation effects on jet noise on sideline, $4^{\text {th }}$ AIAA/CEAS Aeroacoustics Conference, Toulouse, France.

[17] J. L. T. Lawrence, M. Azarpeyvand and R. H. Self 2011, Interaction between a flat plate and a circular subsonic jet , AIAA 2011-2745, $17^{\text {th }}$ AIAA/CEAS Aeroacoustics Conference, Portland, Oregon.

[18] A. V. G. Cavalieri, P. Jordan and Y. Gervais 2014. Scattering of wavepackets by a flat plate in the vicinity of a turbulent jet, Journal of Sound and Vibration, 333, pp. 6516-6531, 2014

[19] K. B. M. Q. Zaman, C. A. Brown and J. E. Bridges 2013, Interaction of a Rectangular Jet with a Flat-plate Placed Parallel to the Flow, AIAA Paper 2013-2184.

[20] C. A. Brown 2014. Developing an Empirical Model for Jet-Surface Interaction Noise. AIAA SciTech 2014, 1317 January 2014

[21] M. S. Howe 1999. Trailing-edge noise at low Mach numbers. Journal of Sound and Vibration, vol. 225 (2), 211-238.

[22] M. E. Goldstein 1978a, Characteristics of unsteady motion on transversely sheared mean flows, J. Fluid Mech. Vol. 89, pp. 433-468.

[23] M. E. Goldstein 1979. Scattering and distortion of the unsteady motion on transver sely sheared mean Flows, J. Fluid Mech. Vol. 91, pp. 601-632.

[24] M. E. Goldstein, M. Z. Afsar and S. J. Leib 2013, Non-Homogeneous Rapid Distortion Theory on Transversely Sheared Mean Flows, Journal of Fluid Mechanics, vol. 736, pp. 532-569.

[25] J. E. Ffowcs Williams and L. H. Hall 1970, Aerodynamic Sound Generation by Turbulent Flow in the Vicinity of a Scattering Half Plane, Journal of Fluid Mechanics, Vol. 40, pp. 657-670.

[26] L. Prandtl 1933, Attaining a steady air stream in wind tunnels. N.A.C.A. Tech. Memo. No. 726.

[27] G. I. Taylor 1935, Turbulence in a contracting stream. 2. Angew. Math. Mech. 15, p. 91.

[28] G. K. Batchelor and I. Proudman 1954. The effect of rapid distortion of a fluid in turbulent motion. Quart. J . Mech. Appl. Math. 7, p. 83.

[29] J. C. R. Hunt, A theory of turbulent flow around two dimensional bluff bodies J. Fluid Mech. Vol. 61, pp. 625-706, 1973.

[30] M. E. Goldstein, Unsteady vortical and entropic distortions of potential flows round arbitrary obstacles, J. Fluid Mech. Vol. 84, 2, pp. 305-329, 1978 b.

[31] M. E. Goldstein, P. A. Durbin, The effect of finite turbulence spatial scale on the amplification of turbulence by a contracting stream, J. Fluid Mech. Vol.98, pp. 473-508, 1980.

[32] J. C. R. Hunt and F. Hussain 1991, A note on velocity, vorticity and helicity of inviscid fluid elements, J. Fluid Mech., vol. 229, p p. 587- 569. 
[33] H. K. Moffatt 1981, Some developments in the theory of turbulence. Journal of Fluid Mechanics, vol. 106, pp. 27-47.

[34] M. E. Goldstein 1984, Aeroacoustics of turbulent shear flows. Annu. Rev. Fluid Mech. 16, 263-286.

[35] A. Khavaran 2014, Acoustics of Jet Surface Interaction - Scrubbing Noise, NASA Technical Report, GRC-EDAA-TN15616.

[36] Advanced Boundary Cartesian Meshing Technology in SolidWorks Flow Simulation, Dassault Systemes SolidWorks Corporation Technical Paper.

[37] Enhanced Turbulence Modelling in SolidWorks Flow Simulation, Dassault Systemes SolidWorks Corporation Technical Paper.

[38] S. J. Leib and M. E. Goldstein 2011, Hybrid Source Model for Predicting High-Speed Jet Noise, AlAA Journal, Vol. 49, No. 7, pp. 1324-1335.

[39] R. Deissler 1998, Turbulent Fluid Motion, Combustion and International Series, CRC Press.

[40] H. Tennekes 1971, J. Lumley, A First course in turbulence, MIT Press, Cambridge, Mass., USA.

[41] M. Gruber and P. F. Joseph 2010, Experimental investigation of airfoil self noise and turbulent wake reduction by use of trailing edge serrations. $16^{\text {th }}$ AIAA/CEAS Aeroacoustics Conference, AIAA 2010-3803.

[42] B. Lyu, M. Azarpeyvand and S. Sinayoko 2015, A Trailing-Edge Noise Model for Serrated Edge, AIAA 20152362, AlAA Aviation 22-26 June, Dallas, TX.

[43] J. Matthews and N. Peake 2015, Noise generation by turbulence interacting with an aerofoil with a serrated leading edge, AIAA 2015-2204, AIAA Aviation 22-26 June 2015, Dallas, TX, USA .

[44] P. Chaitanya, S. Narayanan, P. Joseph, C. Vanderwel, J. Turner, J. W. Kim and B. Ganapathisubramani 2015, Broadband noise reduction through leading edge serrations on realistic aerofoils, AIAA 2015-2202, AIAA Aviation 22-26 June 2015, Dallas, TX.

[45] S. R. Koh, W. Schroder and M. Meinke 2010, Experimental study of noise reduction via wall turbulence control, AIAA 2010-3990, 16 ${ }^{\text {th }}$ AIAA/CEAS Aeroacoustics Conference.

[46] R. K. Amiet 1975, Acoustic radiation from an airfoil in a turbulent stream, Journal of Sound and Vibration, vol. 41, no. 4, pp. 407-420.

[47] S. A. E. Miller 2015. The prediction of noise due to jet turbulence convecting past flight vehicle trailing edges. Applied Acoustics 90 (2015) 42-53

[48] L. Ayton \& N. Peake. 2015. On high-frequency sound generated by gust aerofoil interaction in shear flow. J. Fluid Mech., vol. (766), pp. 297-325. 
[49] William Wolf, Sanjiva Lele,Giridhar Jothiprasad, Lawrence Cheung, "Investigation of Noise Generated by a DU96 Airfoil" $18^{\text {th }}$ AIAA/CEAS conference, June 4-6, 2012, Colorado Springs, CO, AIAA 2012-2055

[50] Lawrence Cheung, Giridhar Jothiprasad, Hao Shen "Large eddy simulation of airfoil self-noise",. Internoise 2012, Aug. 19-22, NYC, NY

[51] P. Venugopal, L. Cheung, G. Jothiprasad, S. K. Lele. "Large eddy simulation of a wind turbine airfoil at high angle of attack", Center for Turbulence Research Proceedings of the Summer Program 2012 (https://web.stanford.edu/group/ctr/Summer/SP12/02.09_venugopal.pdf)

[52] U. Paliath \& S. Premasuthan (2013). Large Eddy Simulation for Jet Installation Effects. AIAA 2013-3137.

[53] M. Roger, S. Moreau and M, Wang 2002, An analytical model for predicting airfoil self-noise using wallpressure statistics, Center for Turbulence Research 405 Annual Research Briefs.

[54] M. Wang 1998, Computation of trailing-edge noise at low Mach number using LES and acoustic analogy, Center for Turbulence Research 91 Annual Research Briefs.

[55] S. Glegg, B. Morin, O. Atassi and R. Reba 2008, Using RANS Calculations of Turbulent Kinetic Energy to Provide Predictions of Trailing Edge Noise, 14th AIAA/CEAS Aeroacoustics Conference (29th AIAA Aeroacoustics Conference) AIAA 2008-2993 5 - 7 May 2008, Vancouver, British Columbia Canada.

[56] M. Karimi, P. Croaker, N. Kessissoglou, C. Doolan and S. Marburg 2014, Self-noise prediction of a sharpedged strut using a quasi-periodic CFD-BEM technique, Inter-Noise 2014, Melbourne, Australia, $16^{\text {th }}-19^{\text {th }}$ November, 2014.

[57] C.A. Albarracin, C.J. Doolan, C.H. Hansen and L.A. Brooks 2011, Turbulent trailing edge noise estimation using a RANS- based statistical noise model, Paper Number 43, Proceedings of ACOUSTICS 2011 2-4 November 2011, Gold Coast, Australia.

[58] M. E. Goldstein, Aeroacoustics, McGraw Hill, New York, 1976.

[59] Goldstein, M. E. 2005. On identifying the true sources of aerodynamic sound. J. Fluid Mech. Vol. 526, pp. $337-347$.

[60] Goldstein, M. E. 2009. A theoretical basis for identifying the sound sources in a turbulent flow. Int. J. Aero. Ac. Vol. 8 (4), pp. 283 - 300.

[61] B. NOBLE, Methods Based on the Wiener Hopf Technique for the Solution of Partial Differential Equations, Pergamon Press, 1958.

[62] D. G. CRIGHTON and F. G. LEPPINGTON 1970, Scattering of aerodynamic noise by a semi-infinite compliant plate. J. Fluid Mech. 43 (40), 721-736.

[63] A.V.G. Cavalieri, W.R. Wolf, P. Jordan and Y. Gervais 2013. Diffraction effects of finite and semi-infinite flat plates in the vicinity of a turbulent subsonic jet. 22nd International Congress of Mechanical Engineering (COBEM 2013) 
[64] M. S. Howe 2007, Hydrodynamics and Sound. Cambridge University Press.

[65] D. A. Russell, J. P. Titlow and Y-J Bemmen 1999. Acoustic monopoles, dipoles, and quadrupoles: An experiment revisited. Am. J. Phys. Vol. 67 (8), pp. 660-664.

[66] W.R. Wolf \& S. K. Lele (2011). Trailing Edge Noise Predictions Using Compressible LES and Acoustic Analogy. AIAA 2011-2784.

[67] J. E. Bridges and C. A. Brown 2005, Validation of the small Hot jet Rig for Jet Noise Research, AIAA Paper 2005-2846, 2005.

[68] A. A. Townsend 1980, The Structure of turbulent shear flow, Cambridge University Press.

[69] Gutmark, E. and Wygnanski, I. 1976. The Planar Turbulent Jet. J. Fluid Mech., vol. 73, pp. 465-495.

[70] Bridges, J. E. and Wernet, M. P. 2013. Turbulence Measurements in Rectangular Jets with Aft Deck. NASA Technical Working Group Meeting.

[71] C.K.G. Lam \& K. Bremhorst. 1983. Modified Form of the k-e Model for Predicting Wall Turbulence. Transactions of the ASME. Vol. 103, pp. 456-460.

[72] R. Mittal \& G. laccarino 2005. Immersed Boundary Methods. Annu. Rev. Fluid Mech. 2005. 37:239-61

[73] V. Dippold, Acoustic Reference Nozzle with Mach 0.97, Unheated Jet Flow, NPARC Alliance Validation Archive, NASA Glenn Research Center, http://www.grc.nasa.gov/WWW/wind/valid/arn/index.html.

[74] M.E. Goldstein \& S. J. Leib 2008. The aero-acoustics of slowly diverging supersonic jets. J. Fluid Mech., vol. (600), pp 291- 337.

[75] V.A. Semiletov, P. G. Yakovlev, S.A. Karabasov, G.A. Faranasov, V. F. Kopiev. 2015. Computational modelling of jet-wing interaction: the effect of asymmetries from the jet inflow conditions and the wing. AIAA 20153129.

[76] W.R. Wolf \& S.K. Lele 2010. Acoustic Analogy Formulations Accelerated by Fast Multipole Method for TwoDimensional Aeroacoustic Problems. AIAAJ, vol. 48, No. 10, pp. 2274-2285.

[77] C. C. Yu, W. R. Wolf and S. K. Lele 2012. Quadrupole Noise in Turbulent Wake Interaction Problems. AIAA 2012-2057.

[78] W.R. Wolf, J-L Azevedo \& S.K. Lele 2012. Convective effects and the role of quadrupole sources for jet aero-acoustics. J. Fluid Mech., vol. 708, pp. 502-538.

[79] W. R. Wolf, B. Backes, E. M. Filho, J-L. F. Azevedo 2013. Investigation of Noise Sources in a TwoDimensional Model Airframe Noise Problem with Wake Interaction. AIAA 2013- 2121.

[80] R. D. Sandberg and N. D. Sandham. 2007. A Modification of Amiet's Classical Trailing Edge Noise Theory for Strictly Two Dimensional Flows. University of Southampton, Report No. AFM-07/04. 
[81] V.A. Semiletov, S.A. Karabasov, A.P. Markesteijn, A. Chintagunta "Empiricism-free noise calculation from LES solution based on Goldstein generalized acoustic analogy: volume noise sources and meanflow effects", AIAA-2015-2536.

[82] Yu.Korolev, S.A. Karabasov, V.Toropov, Automatic "Optimizer vs Human Optimizer for Low-Order Jet Noise Modelling", AIAA-2015-2215.

[83] S. A. Karabasov, M.Z. Afsar, T.P Hynes, A. P Dowling, W. A. McMullan, C. D. Pokora, G. J. Page and J. J. McGuirk 2010, J.J, Jet Noise: Acoustic Analogy Informed by Large Eddy Simulation, AIAA Journal, Vol. 48, No. 7, pp. 1312-1325.

[84] G. A. Campbell and R. M. Foster 1942, Fourier integrals for practical applications, Bell Telephone Laboratories.

[85] M. Abramowitz and I. Stegun 1970, Handbook of Mathematical Functions, Dover Publications.

[86] M. J. Lighthill 1964, Fourier Analysis and Generalized Functions. Cambridge University Press. 